US Army Corps

of Engineers ${ }_{\circledast}$

Engineer Research and

Development Center

ERDC 6.2/6.3 Military Engineering (ME) RAFTER

\title{
Using EASEE's Acoustical Calculations in MATLAB
}

D. Keith Wilson, Ross E. Alter, Katrina M. Burch, and

Michelle E. Swearingen

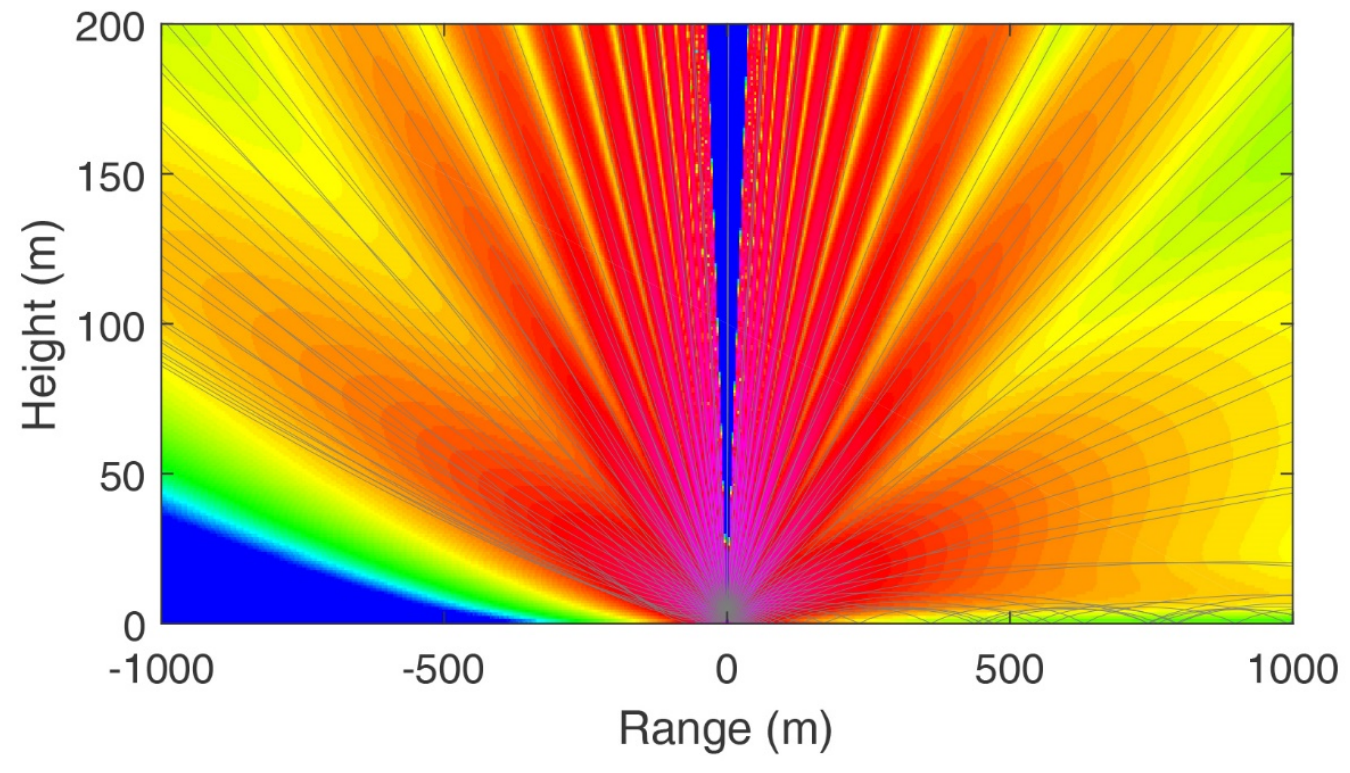

30

40

50

60

70

80

90

Transmission loss $(\mathrm{dB})$ 
The U.S. Army Engineer Research and Development Center (ERDC) solves the nation's toughest engineering and environmental challenges. ERDC develops innovative solutions in civil and military engineering, geospatial sciences, water resources, and environmental sciences for the Army, the Department of Defense, civilian agencies, and our nation's public good. Find out more at www.erdc.usace.army.mil.

To search for other technical reports published by ERDC, visit the ERDC online library at http://acwc.sdp.sirsi.net/client/default. 


\section{Using EASEE's Acoustical Calculations in MATLAB}

D. Keith Wilson, Ross E. Alter, and Katrina M. Burch

U.S. Army Engineer Research and Development Center (ERDC)

Cold Regions Research and Engineering Laboratory (CRREL)

72 Lyme Road

Hanover, NH 03755-1290

Michelle E. Swearingen

U.S. Army Engineer Research and Development Center (ERDC)

Construction Engineering Research Laboratory (CERL)

2902 Newmark Dr.

Champaign, IL 61822

Final Report

Approved for public release; distribution is unlimited.

Prepared for Headquarters, U.S. Army Corps of Engineers

Washington, DC 20314-1000

Under ERDC 6.2/6.3 Military Engineering (ME), Remote Assessment of Infrastructure for Ensured Maneuver (RAFTER), funded by 62784/T40/46, "Propagation Effects" 


\section{Abstract}

EASEE (Environmental Awareness for Sensor and Emitter Employment) is a Java-based software framework for modeling the impacts of the weather and terrain on signal propagation and sensor performance.

EASEE includes extensive capabilities for representing the environment (atmosphere, land cover, terrain elevation, and soil properties), along with many different models related to acoustic, optical, radio frequency, and seismic signals. This report describes how to run EASEE from MATLAB, which is a popular software package for performing numerical calculations and displaying graphics. For this purpose, a simple installation configuration and MATLAB script were devised to set up and initialize EASEE. The focus of the report is on using EASEE for acoustic propagation calculations, which is its most mature signal modality. The report describes two general approaches to performing acoustical calculations in EASEE: one that involves using EASEE for its environmental representation only and then running the acoustic propagation calculation using a MATLAB toolbox and a second that uses EASEE for both its environmental layer and the acoustical calculation. Overall, this report shows that the MATLAB user interface provides convenient access to EASEE's powerful signal modeling capabilities.

DISCLAIMER: The contents of this report are not to be used for advertising, publication, or promotional purposes. Citation of trade names does not constitute an official endorsement or approval of the use of such commercial products. All product names and trademarks cited are the property of their respective owners. The findings of this report are not to be construed as an official Department of the Army position unless so designated by other authorized documents. 


\section{Contents}

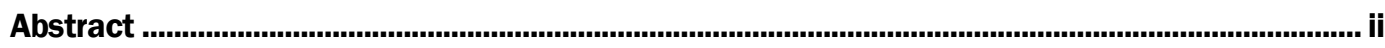

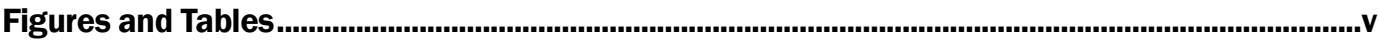

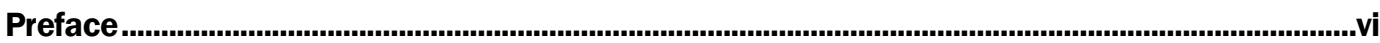

Acronyms and Abbreviations ........................................................................................................

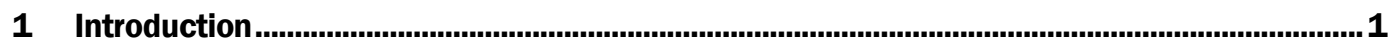

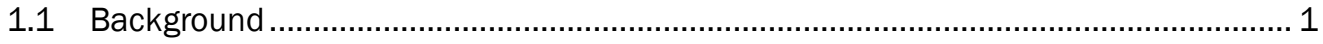

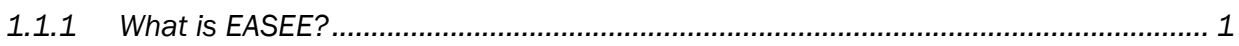

1.1.2 EASEE software design and capabilities ................................................................ 2

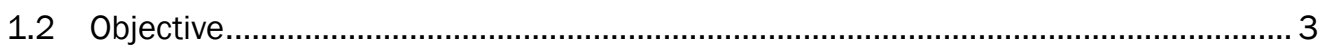

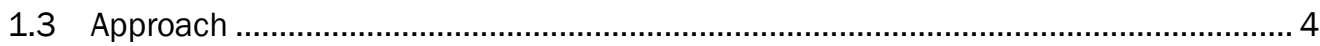

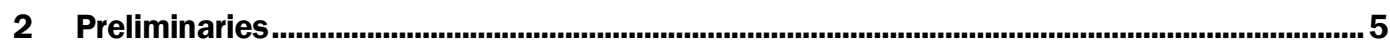

2.1 Installing the EASEE files............................................................................ 5

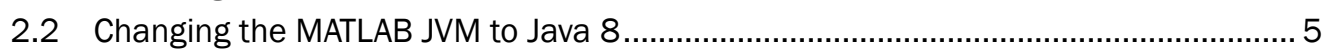

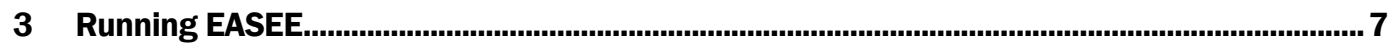

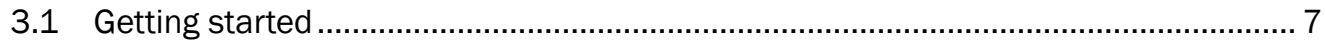

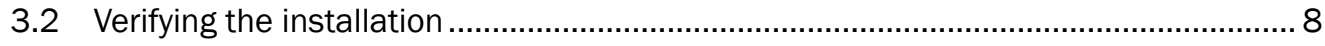

3.3 Simple example .......................................................................................... 8

3.4 Some useful background ............................................................................... 10

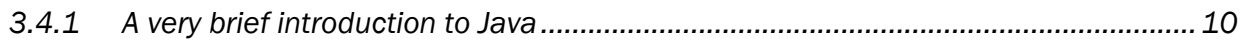

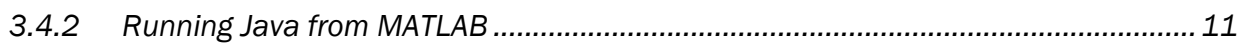

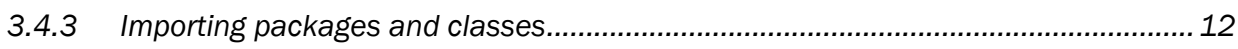

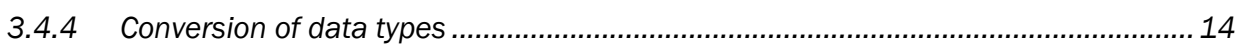

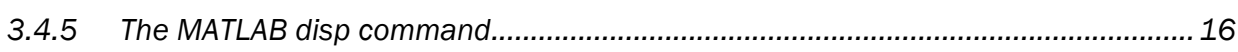

3.4.6 Java enumerations and inner classes ............................................................... 16

4 Geographic Coordinates, Grids, and Elevation Maps .......................................................21

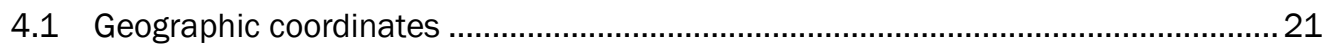

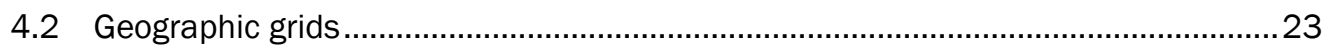

4.3 Digital elevation, surface, land cover, and soil grids .......................................... 24

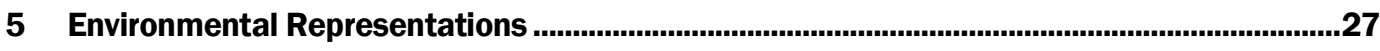

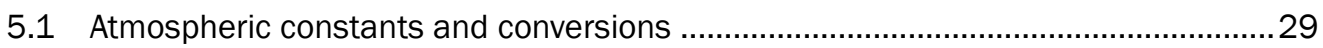

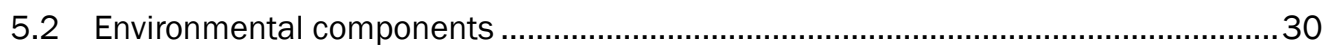

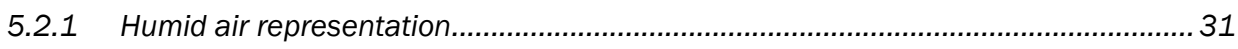

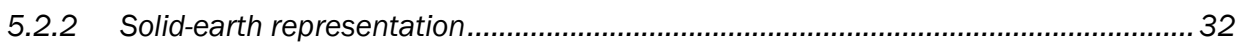

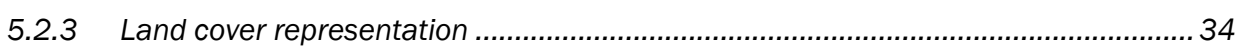

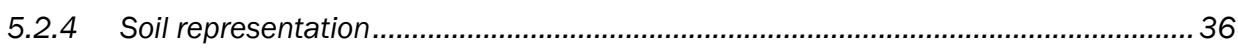

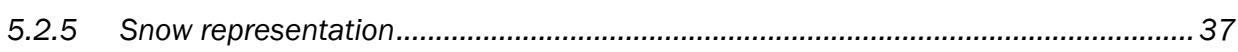

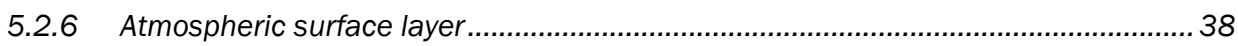




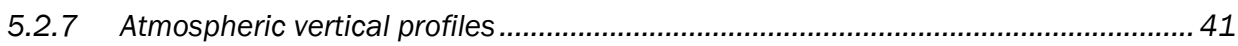

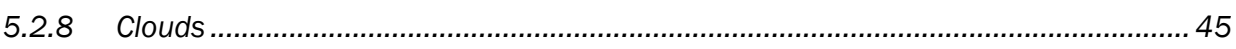

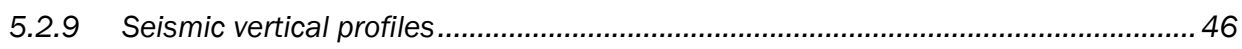

5.3 Environmental representations........................................................................ 48

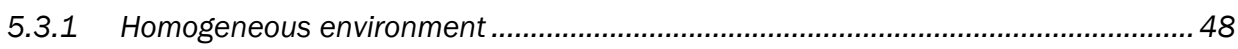

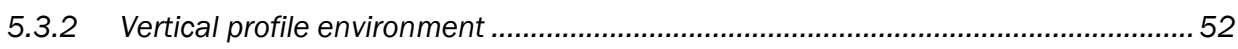

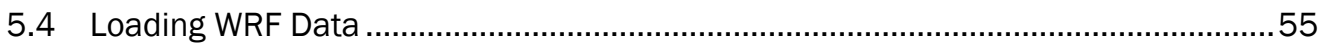

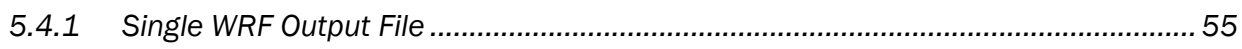

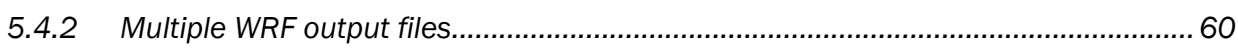

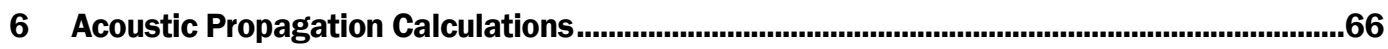

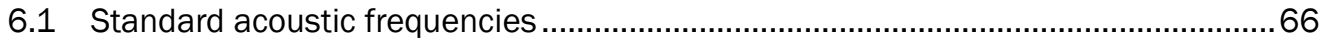

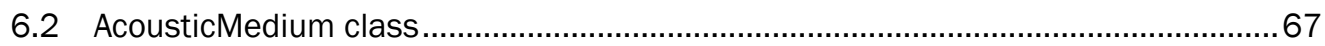

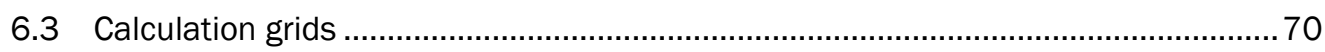

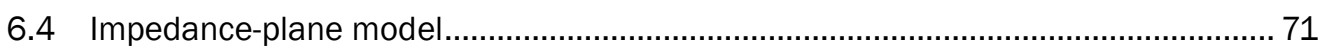

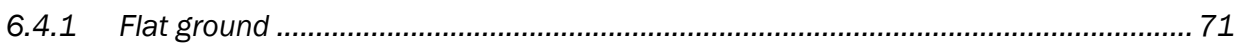

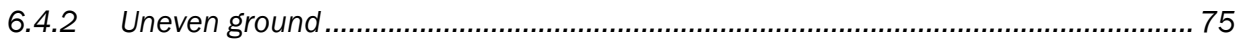

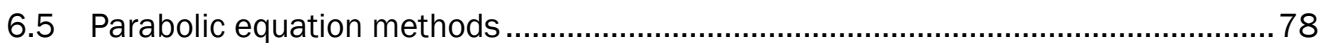

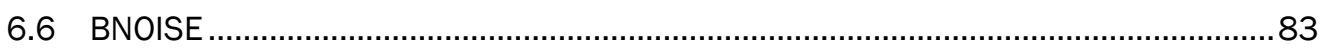

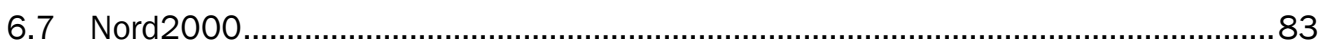

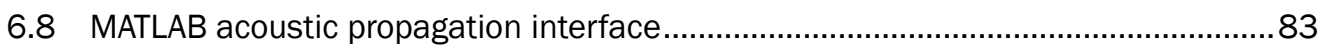

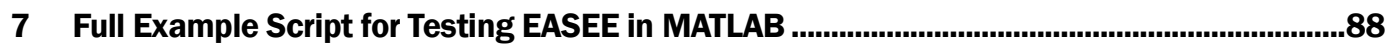

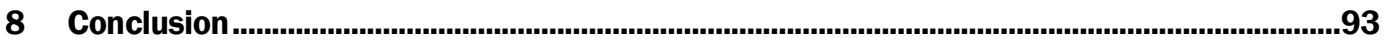

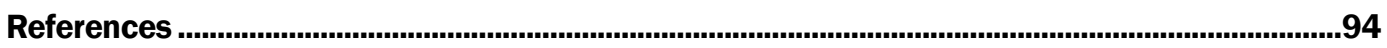

Report Documentation Page 


\section{Figures and Tables}

\section{Figures}

1 EASEE framework 3

2 Output from the methodsview('HumidAir') command in MATLAB .....................................14

3 Hierarchy of EASEE's environmental classes. Phrases in bold are the main environmental classes within EASEE, and italicized phrases are subclasses of the foremost environmental class-EnvironScenario. All other listings are fields (e.g., friction velocity is a field within AtmosSfcLayer, which is a field within AtmosOneDim, which is a field within EnvironVertProf). All phrases with only capitalized terms (e.g., EnvironVertProf) are instances of classes. Blue font color indicates recent additions

4 Vertical profiles of temperature, specific humidity, and wind speed as created by specification of atmospheric surface layer properties for a moderately windy, unstable case.

5 Vertical profiles of air temperature (blue), the eastward wind component (red), and the northward wind component (orange) derived from a WRF test file in the EASEE repository.

6 Vertical profiles of air temperature derived from three pseudoensemble test WRF files in the EASEE repository.

7 Attenuation coefficient for air at $20^{\circ} \mathrm{C}, 40 \%$ relative humidity, and sealevel pressure

8 Transmission loss (TL) for propagation at a frequency of $100 \mathrm{~Hz}$ over several different ground surfaces.

9 Terrain elevations (digital elevation model, or DEM) used for the transmission loss calculation shown in Fig. 10. The coordinate axes are the easting and northing relative to the southwest corner of the domain

10 Transmission loss (TL) calculation for a source in hilly terrain. The frequency is $100 \mathrm{~Hz}$. The source is positioned at an easting of $7 \mathrm{~km}$ and a northing of $5 \mathrm{~km}$. The DEM for the calculation is shown in Fig. 9

11 Comparison of transmission loss (TL) calculations by several different codes

12 Transmission loss resulting from a wide-angle parabolic equation calculation using the MATLAB interface

13 Same as Fig. 12 but using atmospheric output from the WRF weather model.

14 Difference in transmission loss (TL) between Fig. 13 and Fig. 12 


\section{Preface}

This study was conducted for the Assistant Secretary of the Army for Acquisition, Logistics, and Technology (ASA[ALT]) under the U.S. Army Engineer Research and Development Center (ERDC) 6.2/6.3 Military Engineering (ME), Remote Assessment of Infrastructure for Ensured Maneuver (RAFTER) program, funded by $62784 / \mathrm{T} 40 / 46$, under the "Propagation Effects" pillar. The technical monitor was Ms. Danielle Whitlow, Program Manager for RAFTER.

The work was performed by the Signature Physics Branch (CEERD-RRD) of the Research and Engineering Division (CEERD-RR), ERDC Cold Regions Research and Engineering Laboratory (CRREL), and the Ecological Processes Branch (CEERD-CNN) of the Installations Division (CEERD$\mathrm{CN}$ ), ERDC Construction Engineering Research Laboratory (CERL). At the time of publication, Dr. Andrew Niccolai was Chief, CEERD-RRD; Mr. J. D. Horne was Chief, CEERD-RR; Dr. Chris Rewerts was Chief, CEERDCNN; and Ms. Michelle Hanson was Chief, CEERD-CN. The Deputy Director of ERDC-CRREL was Mr. David B. Ringelberg, and the Director was Dr. Joseph L. Corriveau. The Deputy Director of ERDC-CERL was Dr. Kirankumar V. Topudurti, and the Director was Dr. Lance D. Hansen.

COL Ivan P. Beckman was Commander of ERDC, and Dr. David W. Pittman was the Director. 


\section{Acronyms and Abbreviations}

$1-\mathrm{D}$

2-D

3-D

API

ARW

ASA(ALT)

ASL

BNOISE

CERL

CNPE

CRREL

DEM

DSM

EASEE

ERDC

FASST

FFP

GFPE

IR

.jar

JDK

JRE
One-Dimensional

Two-Dimensional

Three-Dimensional

Application Programming Interface

Advanced Research WRF

Assistant Secretary of the Army (Acquisition, Logistics, and Technology)

Atmospheric Surface Layer

Blast Noise

Construction Engineering Research Laboratory

Crank-Nicholson Parabolic Equation

U.S. Army Cold Regions Research and Engineering Laboratory

Digital Elevation Model

Digital Surface Model

Environmental Awareness for Sensor and Emitter Employment

U.S. Army Engineer Research and Development Center

Fast All-Season Strength

Fast-Field Program

Green's Function Parabolic Equation

Infrared

Java Archive file

Java SE Development Kit

Java SE Runtime Environment 


$\begin{array}{ll}\text { JVM } & \text { Java Virtual Machine } \\ \text { KNEE } & \text { KNEE is Not EASEE in Its Entirety } \\ \text { ME } & \text { Military Engineering } \\ \text { MGRS } & \text { Military Grid Reference System } \\ \text { MOST } & \text { Monin-Obukhov Similarity Theory } \\ \text { NASA } & \text { National Aeronautics and Space Administration } \\ \text { NCAR } & \text { National Center for Atmospheric Research } \\ \text { NetCDF } & \text { Network Common Data Format } \\ \text { NLCD } & \text { National Land Cover Dataset } \\ \text { P- } & \text { Compressional } \\ \text { UTC } & \text { Thiversal Transverse Mercator } \\ \text { PE } & \text { Parabolic Equation } \\ \text { RAFTER } & \text { Remote Assessment of Infrastructure for Ensured Maneuver } \\ \text { RF } & \text { Radio Frequency } \\ \text { S- } & \text { Shear }\end{array}$




\section{Introduction}

\subsection{Background}

\subsubsection{What is EASEE?}

EASEE (Environmental Awareness for Sensor and Emitter Employment) provides a versatile software framework for modeling terrain and weather impacts on signal propagation and the performance of battlefield sensors. Two previous reports (Wilson et al. 2009; Wilson and Yamamoto 2014) describe the overall design and many technical aspects of EASEE. As Wilson and Yamamoto (2014) explain,

The performance and utility of battlefield and homeland security sensors depends on many complex environmental and mission-related factors. This is generally true whether the sensors are ground-based or airborne; whether the sensors are acoustic, seismic, optical, infrared (IR), radio frequency (RF), or magnetic; and whether the observable features of signal emitters originate from vehicles, humans, or electronic equipment. Realistic modeling and simulation of environmental factors can improve the effectiveness of mission planning and can further the development of more effective sensor system designs and doctrine for their usage.

The same report also summarizes the motivation underlying EASEE's software design and the history of its development:

The primary design goal in developing EASEE was to create a highly reusable software framework, which would provide realistic, physics-based simulations of terrain and weather impacts on all types of battlefield signals and sensors. EASEE is a successor to the SPEBE (Sensor Performance Evaluator for Battlespace Environments) software (Wilson et al. 2002), which had become widely used but accommodated only acoustics and seismics. SPEBE was also written in MATLAB, which limited options for interfacing it with other simulations and mission planning tools. The EASEE project began as an ERDC [U.S. Army Engineer Research and Development Center] applied research work package in 2006. It involved a complete adaptation of the capabilities of SPEBE into the Java program- 
ming language, to make the code more reusable, and a new, object-oriented modeling paradigm that could accommodate signal modalities in addition to acoustic and seismic.

Thus EASEE, a Java-based code, was the successor to SPEBE, a MATLABbased code. The motivation for making this transition, as described above, remains valid. Largely because EASEE is written in Java, it has been successfully integrated into many different user interfaces and deployed as a web service. However, there are situations, especially in research applications, where it is nonetheless desirable to access EASEE's modeling capabilities from MATLAB. This turns out to be very feasible, since Java can be directly called from MATLAB. From a programming perspective, EASEE appears to function in essentially the same manner as native MATLAB code. This report will describe how to use EASEE from MATLAB. First, however, it is useful to outline some aspects of EASEE's design and capabilities.

\subsubsection{EASEE software design and capabilities}

EASEE consists of two main components-EASEELib, which is the militarily sensitive and limited distribution parts of EASEE, and KNEE, which contains the nonsensitive parts. KNEE is short for "KNEE is Not EASEE in

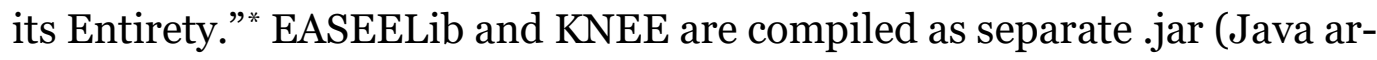
chive) files. KNEE can run by itself, but EASEELib requires KNEE to run.

Figure 1 shows the basic software design of EASEE. The design involves a sequence of interlinked modeling stages, from generation of the signal to propagation through the environment, sensing of the signal, and processing. Each step in the sequence is called a framework element. An advanced Java technique called generics enforces compatibility between framework elements and data passed between them. The different models thus always fit together as designed.

\footnotetext{
* The KNEE acronym is an homage to the acronym for the well known GNU software project, which means "GNU is Not Unix."
} 
Figure 1. EASEE framework.

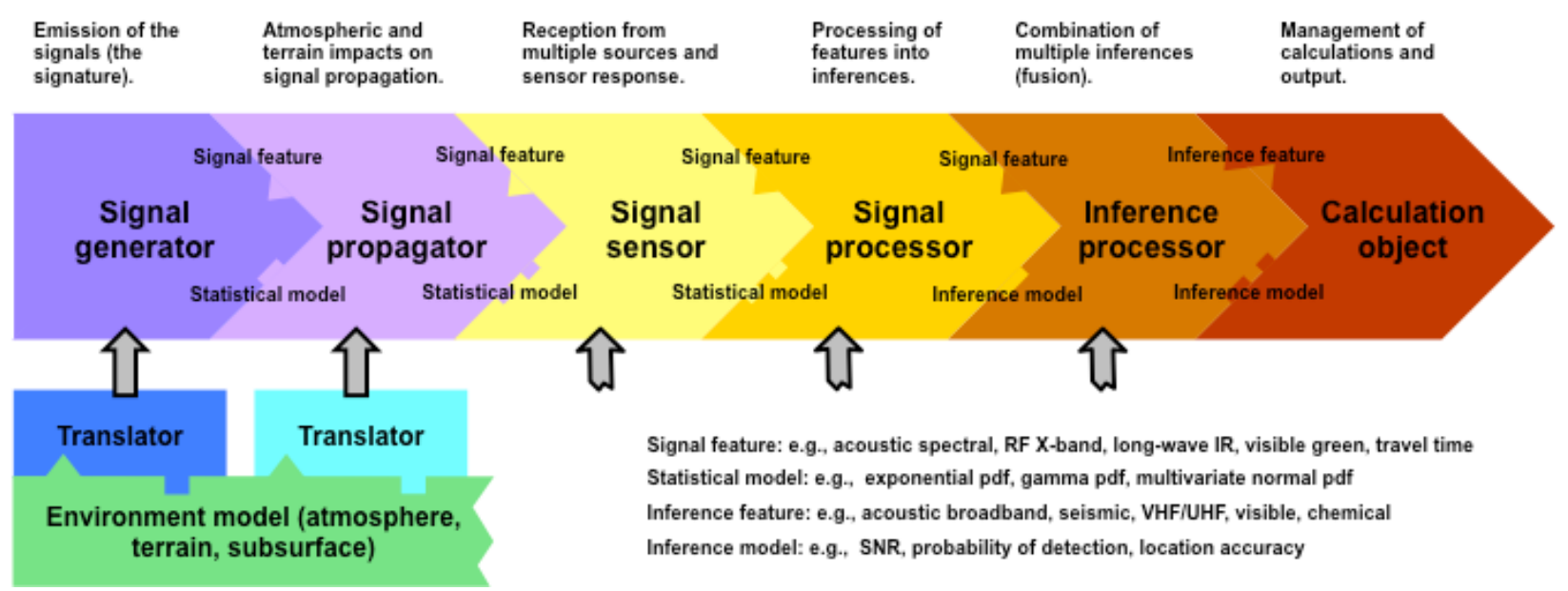

EASEE presently incorporates modeling capabilities related to many different signal modalities, including acoustic, seismic, RF, visible, IR, and chemical/biological. Additionally, EASEE includes its own detailed representations of the environment, by which we mean primarily the atmosphere, land cover, terrain elevation, and subsurface properties. Each model implemented as a framework element has its own class for translating environmental representations to parameters needed by the model. EASEE's environmental representation is an example of a data abstraction layer. (For a description of data abstraction layers, see, for example, Rouse 2014.) The purpose of this layer is to isolate the modeling capabilities from the environmental representation. Suppose we wish to interface a new environmental data resource to EASEE (e.g., a new atmospheric forecast model or a new land cover scheme). We thus would need to write new code that translates the data resource into EASEE's native environmental representation. But this only needs to happen once-then the new data resource will become available to all models in EASEE without modifying any code associated with the individual models or their parameter translation classes.

\subsection{Objective}

The primary purpose of this report is to provide an easy-to-use, step-bystep manual for those interested in using EASEE to model outdoor sound propagation. Specifically, our report shows how to run EASEE within MATLAB, a programming platform that is widely used among potential users of EASEE. 


\subsection{Approach}

EASEE is too extensive to practically describe here all of its components and how they can be called from MATLAB. Rather, this report focuses on some particular aspects of setting up the environmental representations and running acoustic propagation models. The report also focuses on $d y$ namic methods (those involving object instantiation) in EASEE, as opposed to static methods. In general, much of the functionality described here (such as humidity conversions, calculation of absorption coefficients, and calculation of solid material properties) is supported by static methods also. We do not attempt here to describe all the methods (static or dynamic) that are available in EASEE. Programmers may find additional useful information by examining the constructors and other methods in the original Java code (which are generally well documented) or by reading the EASEELib and KNEE Javadoc (computer-generated HTML documentation). The test packages in EASEELib and KNEE are also very helpful in providing examples of how to call the code.

This report is intended primarily for acousticians and software developers who wish to use EASEE to model outdoor sound propagation. However, it may also be of interest to developers desiring an introduction to the functionality of EASEE, complete with useful examples and test cases.

In this report, we first discuss some preliminaries about setting up MATLAB and installing EASEE. Next, we discuss the various classes in EASEE for representing the environment, by which is meant the atmosphere, surface state, and subsurface (geology). Lastly, we discuss how to run propagation calculations in EASEE. 


\section{Preliminaries}

In this section, we describe the preliminary steps that the user must perform to run EASEE from MATLAB, beginning with how to configure the Java Virtual Machine (JVM) and install the EASEE .jar files.

\subsection{Installing the EASEE files}

The files needed to run EASEE from MATLAB are distributed in the file easee-repo.zip, which is available from the authors of this report. Place this file in a convenient location and then unzip the contents. You should see a few files and several directories, including air, CNPE, and EASEEInterface. If you navigate to the EASEEInterface directory, you will find a folder called easee-repo, and a number of other files, the most essential being StartEASEE.m, SetPaths.m, RunCalc.m, PlotCalc.m, createMATLABShortcut.m, and Rainbow.mat. In particular, the file StartEASEE.m automatically performs a number of functions related to configuring paths, thus simplifying the process of running EASEE from MATLAB. Also, the file TestEASEE. $m$ contains all of the executable MATLAB code in this technical report (except for section 7, which is included in CalcExampWithWRF.m) and can be run sequentially (section by section) or all at once so users do not have to manually copy all of the code into MATLAB for testing purposes.

The folder easee-repo contains a number of .jar and executable files. These include the KNEE and EASEELib .jar files, as well as a number of other .jar files that are needed by EASEE.

The next subsection describes the final prerequisite to launching EASEE.

\subsection{Changing the MATLAB JVM to Java 8}

EASEE is compiled using Java version 8. As such, running EASEE from MATLAB requires configuring MATLAB to use a Java $8 \mathrm{JVM}$, rather than Java 7, which is the default JVM distributed with MATLAB as of version R2017a. When MATLAB is originally installed, its JVM is separate from the one by the operating system (Windows, MacOS, or Unix). To determine which Java version is installed on your operating system, type the following into a command prompt: 
The output should look like java version "1.8.0..." if Java 8 is installed.

Users who do not have Java 8 running on their operating system will need to download and install either the Java SE Runtime Environment (JRE) or, if they wish to develop EASEE, the Java SE Development Kit (JDK, which contains the JRE) from http://www.oracle.com/technetwork/java/javase/downloads (Oracle, n.d.).

Once a Java $8 \mathrm{JVM}$ has been installed on the target computer, it is still necessary to point MATLAB to this JVM. The procedure depends on which operating system you are using.

Instructions for Windows users: Create a MATLAB_JAVA environmental variable and set it to the JRE folder, as described at MathWorks (2018a). You will need to restart MATLAB.

Instructions for MacOS users: Several options are provided at MathWorks (2018b). The one we found to be most satisfactory is running the script createMATLABShortcut. For convenience, this script is included in the installation distribution. To use it, navigate to the EASEEInterface folder, and then type

createMATLABShortcut ( ' / Library/Java/JavaVirtualMachines /

jdk1.8.0_111.jdk/Contents/Home/jre')

(Substitute the location for the desired JRE in the quotes.) This will create a new desktop shortcut that will start MATLAB but with the specified JRE. We recommend that you retain this shortcut as well as the MATLAB icon on the Dock, which will start with the MATLAB default JVM as before.

All users: Now, if you restart MATLAB and type ver at the MATLAB prompt, you should see the desired version of the JVM listed. Note that you will need to repeat the preceding instructions (Windows or MacOS) if you install a new JVM.

Having installed EASEE, you are now ready to run it, as described in the next section. 


\section{Running EASEE}

\subsection{Getting started}

The following three simple steps set the stage for running EASEE from MATLAB:

1. Launch MATLAB (e.g., by double-clicking on the desktop icon).

2. Navigate to the EASEEInterface folder (where StartEASEE.m resides), using either the cd command at the MATLAB prompt or the "Browse for Folder" button at the top of the command window.

3. At the MATLAB prompt, type

StartEASEE ('easee-repo')

where easee-repo is the folder containing the EASEE/KNEE jar files. Or, if the easee-repo folder is a subfolder within the current one (as is the case with the normal file distribution), just type the following:

StartEASEE

The StartEASEE function will add these files to MATLAB's Java class path (which you can verify by typing $j$ avaclasspath at the MATLAB prompt) and will attempt to call EASEE for the first time. If you see the following error,

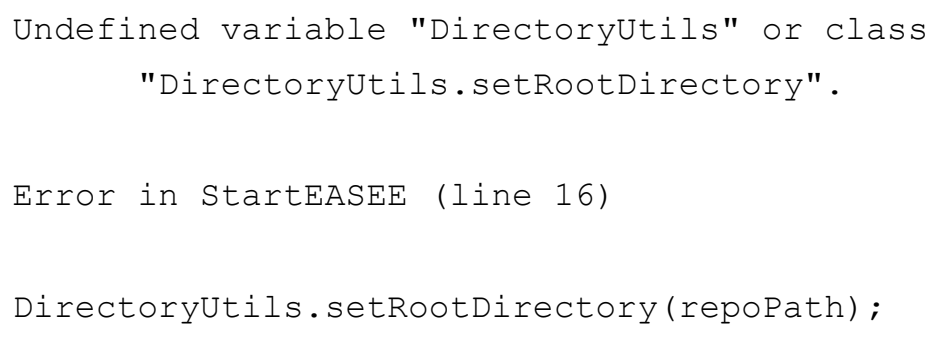

then MATLAB could not find the .jar files. The likely cause is that the name of the folder was incorrectly specified to StartEASEE. The same error will be issued when a version of Java other than Java 8 is running. 


\subsection{Verifying the installation}

Next, type the following six lines at the MATLAB prompt. If they execute without error, then the EASEE and KNEE files have been properly installed:

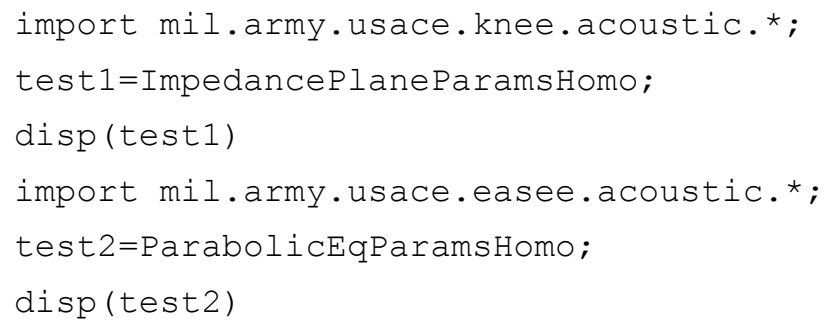

As output, you should see

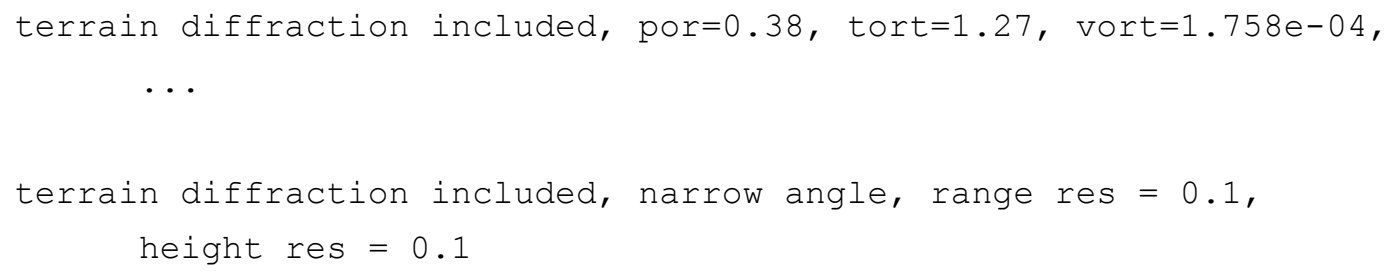

You are now running EASEE!

\subsection{Simple example}

Let us consider a simple, but useful, example in which we calculate the sound pressure above a rigid plane. When entering the following code, it is important to remember that both MATLAB and Java are case sensitive.

First, we import the Java packages needed for the calculation by entering the following at the MATLAB prompt:

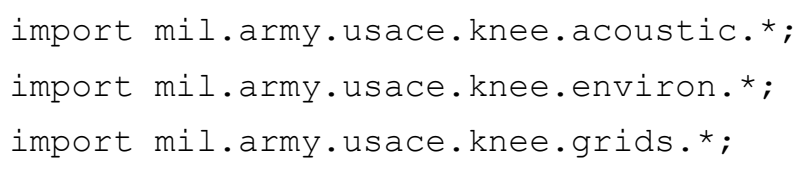

Next, we will set the frequency, source height, receiver height, and range (horizontal distance):

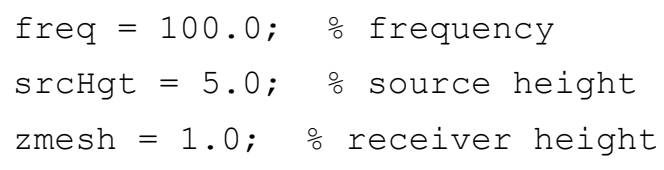


rmesh $=0.0: 100.0: 1000.0 ; \quad$ o horizontal ranges for output

The following sequence of commands sets up the parameters for the impedance-plane model:

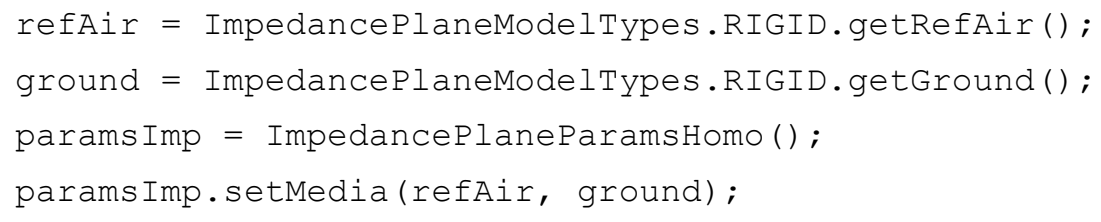

Next, we create an impedance-plane calculation model with the parameters specified above:

impp = ImpedancePlaneModel (params Imp) ;

Lastly, we set up the grid for the calculation and run the model:

tgv = TransmitGridVert (srcHgt, zmesh, rmesh);

calcVals = impp.calcTransGridstruct (freq, tgv);

Now we can display the results of the calculation:

$\operatorname{disp}(10 * \log 10($ calcVals $))$

which yields transmission loss relative to the level that would be observed at $1 \mathrm{~m}$ in free space. Note that EASEE calculates the mean-square sound pressure; so in the preceding, we converted to decibels by multiplying by 10 rather than by 20 . The following values will be displayed:

-17.4677
-34.1577
-40.2729
-43.9186
-46.5455
-48.6131
-50.3266
-51.7957
-53.0857
-54.2391
-55.2846 
The complete code for this example can be found in the script CalcExampImp.m, which is included in the installation distribution.

\subsection{Some useful background}

This section provides some background information on Java and MATLAB that is useful for understanding how to employ EASEE.

\subsubsection{A very brief introduction to Java}

Here we provide a very brief introduction to Java for readers of this report who might be familiar with MATLAB but not with Java. The main purpose is to define Java terminology, such as objects, classes, methods, and constructors. For readers interested in a full tutorial on Java, we would recommend the book Head First Java (Sierra and Bates 2005) for those who are learning Java as their first object-oriented language; Thinking in Java (Eckel 2006) for those who have already learned an object-oriented language; and Java All-in-One Desk Reference for Dummies (Lowe and Burd 2007) for a more complete reference. There are also many excellent, free, online resources, such as the IBM Introduction to Java programming course found at Perry (2010).

Java is a general-purpose, object-oriented programming language and was designed to be simple and reusable on different computer architectures. As the name would suggest, the focus of object-oriented programming is on the concept of objects as opposed to procedures or functions, which was the dominant programming paradigm in popular programming languages such as BASIC and FORTRAN, which preceded Java.

Objects include attributes (data) and methods (procedures) that manipulate the attributes of the object. Objects are typically constructed (instantiated) and then are dynamically modified during program execution. Methods that change the properties of an object are commonly called setter methods; methods that retrieve existing properties are getter methods.

Java also supports the procedural or functional programming through static, as opposed to instance, methods. The static methods do not require construction of an object and can be thought of in the conventional sense of receiving an output for a set of inputs. For some programming problems, static methods arguably provide a more natural solution than objectoriented programming. 
Java objects are described by classes. The class defines fields, which represent the attributes of the object. The fields may be simple data types, such as integers, floating point values, and Strings, or they may be other objects. The class also defines the instance methods for the object. Static methods may also be defined, but these exist independently of the construction of objects of the class.

Typically, classes are defined in a hierarchy, with parent classes and subclasses, or extensions. For example, a class for animals might be defined, a subclass of which could be cats, which might have subclasses for lions, tigers, and housecats.

\subsubsection{Running Java from MATLAB}

Since Java can be directly called from MATLAB, the EASEE Java code is called very similarly to native MATLAB functions. The main differences are that the desired Java packages must be imported and that many of the operations produce or operate on Java objects, rather than on MATLAB native data types. The Java objects generally cannot be directly manipulated (e.g., retrieving, setting, and performing operations on their properties) within MATLAB; the objects must be manipulated by calling the EASEE Java methods from MATLAB.

Java code, when called from within MATLAB (as opposed to when it is called directly from Java), appears essentially the same as the original Java, with a few notable differences:

1. Data types: The data types are not explicitly declared (since MATLAB, unlike Java, is not a type-controlled language). Hence many data conversions are performed automatically according to the MATLAB application programming interface (API). This topic will be discussed later in its own subsection.

2. Object construction: The new keyword in Java is omitted in MATLAB when calling a constructor method. Alternatively, one can construct a Java object with MATLAB's $j$ avaMet hod function, but this is only necessary in some special cases as described in the MATLAB documentation.

3. Invoking Java methods: Just as in Java, static methods are called by specifying the name of the class, followed by a period and then the name of the method. Instance methods are called the same way, with the name of a 
valid object replacing the class name. Alternatively, the javaMethod function can be used, but this is usually unnecessary. If there are no arguments to a method, MATLAB allows the empty parentheses to be omitted.

\subsubsection{Importing packages and classes}

The MATLAB import command, like the Java import statement, provides a way to abbreviate the names of packages and classes. For example, KNEE's geo package has a class called Geocoord, which represents geographic coordinates. To construct a geographic coordinate and assign the result to the variable gc, one could either type

$g_{c}=$ mil.army.usace. knee.geo.GeoCoord $(33.0,-62.2)$;

where the two numbers represent the latitude and longitude in degrees, respectively, or

import mil.army.usace. knee.geo.GeoCoord;

$g_{c}=$ GeoCoord $(33.0,-62.2)$;

A wild card (asterisk) can also be used to import all classes in a Java package. Thus the following will also work:

import mil.army.usace.knee.geo. *;

$g_{c}=$ GeoCoord $(33.0,-62.2)$;

MATLAB does not recommend this last version of the import command due to the potential for creating conflicts in the name space. For example, if one of the imported Java classes implied by the wildcard is named "close", it could conflict with the MATLAB close function.

However, it is important to note that StartEASEE must be executed at the MATLAB command prompt before MATLAB is able to import individual classes in EASEE. Otherwise, if StartEASEE is included in a script that also contains an import command for an individual class (e.g., import mil. army. usace. knee.geo.GeoCoord), then MATLAB will produce an error. Therefore, to avoid confusion (and for convenience), we will use the wildcard version of the import command in the following examples. 
After a package has been imported, one can list the methods within a Java class that is located within that package (such as HumidAir within mil.army . usace.knee.environ) by using methods ('class'), for example

methods ('HumidAir')

which displays

Methods for class HumidAir:

$\begin{array}{llll}\text { HumidAir } & \text { getDewPoint } & \text { getSpecHumDef } & \text { notify } \\ \text { thermCond } & \text { convHum } & \text { getMixRat } & \text { getTemp } \\ \text { notifyAl } & \text { toString } & \text { convRelHumToSpecHum } & \text { getMolHum } \\ \text { getTempCelsius } & \text { prandtl } & \text { viscosity } & \text { convSpecHumToRelHum } \\ \text { getPress } & \text { getTempDef } & \text { pressit } & \text { wait } \\ \text { density } & \text { getPressAtm } & \text { getTempFahr } & \text { setPress } \\ \text { waterVaporPressure } & \text { equals } & \text { getPressDef } & \text { getTempKelvin } \\ \text { setPressAtm } & \text { getCaveats } & \text { getPressMbar } & \text { getThermCond } \\ \text { setPressMbar } & \text { getClass } & \text { getRelHum } & \text { getViscosity } \\ \text { setSpecHum } & \text { getDensity } & \text { getSpecHum } & \text { hashCode } \\ \text { setTemp } & & & \end{array}$

To find out more information about these methods, one can use methodsview ('class'). For example, typing

methodsview ('HumidAir')

results in the following popup window (Figure 2), which shows the name, return type, qualifier (e.g., static), and arguments for each method in the HumidAir class.

The package containing the desired class should be imported before typing methods or methodsview in the MATLAB command prompt. If not, then the entire package name needs to be specified in the MATLAB command prompt, for example: 
Figure 2. Output from the methodsview('HumidAir') command in MATLAB.

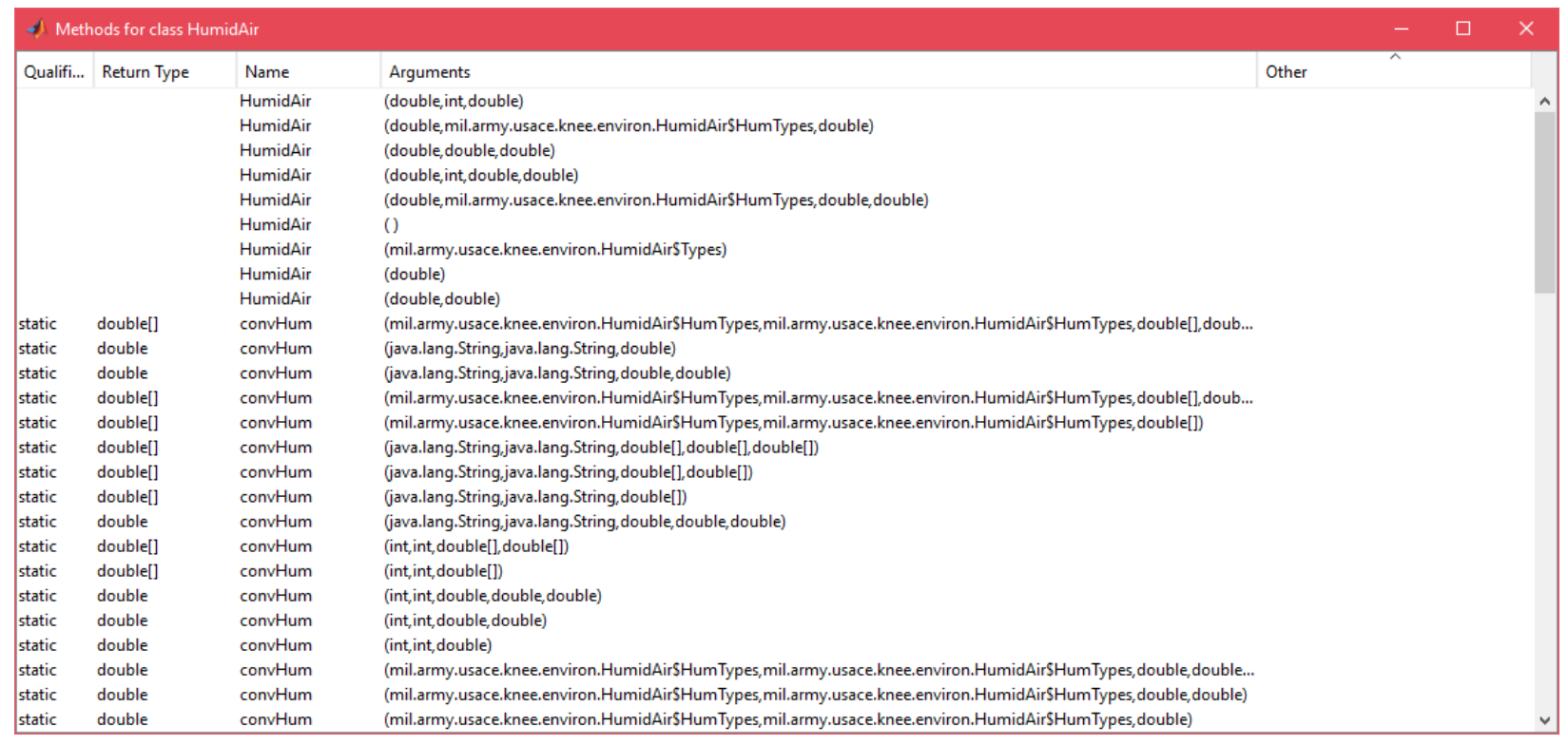

\subsubsection{Conversion of data types}

The MATLAB interface to Java performs a number of automatic conversions between MATLAB and Java data types. These are fully described in the MATLAB help page for "Pass Data to Java Methods." The main thing to keep in mind is that MATLAB's basic data type for variables is a two-dimensional (2-D) array of complex numbers that may, in practice, hold scalar data, a one-dimensional (1-D) array, or a 2-D array. The values may be integer, real, or complex. Hence, when there are overloaded Java methods (methods with the same name) having the same number of arguments but with different types, MATLAB must choose which method is the closest match to a specified argument list. The process involves internal rules for selecting the most appropriate Java method at runtime.

In practice, the rules used by MATLAB usually lead to intuitive results. In particular, if a MATLAB variable holds a real-valued scalar, MATLAB will first search for a Java method with an argument of the Java type double. If such a method does not exist, it will search for a method with type float, then type long, then type int, and so forth. Analogously, the Java float type corresponds to MATLAB single; thus MATLAB will attempt to find a method with Java type float given a variable of type single.

The Java int type corresponds to the MATLAB int 32 type, whereas long corresponds to int 64 . Thus, if the programmer's intent is to call a Java method with argument of type int and there are overriding methods that 
take precedence, the variable must be first converted to the MATLAB type int32.

A MATLAB variable of type logical will be mapped to a Java boolean if possible. MATLAB char maps to a Java string.

Other nonsimple Java data types (i.e., Java objects) are not converted by MATLAB. Rather, MATLAB retains a reference to the Java object, just as Java would.

As a rather contrived example, suppose we have a Java class that defines two methods called add, as follows:

public double add (double $x$, double y)

public int add(int $x$, int $y$ )

Suppose we then set

$\operatorname{val1}=3.69 ;$

$\operatorname{val2}=4.2 ;$

If we then enter

disp (add (vall, val2))

MATLAB will call the add method for double arguments, as expected. But, what happens if we instead set

$\operatorname{val1}=3 ;$

$\operatorname{val2}=4 ;$

Since MATLAB automatically stores these values in its native double-precision floating-point format, the preceding disp command will automatically call the Java add method for double arguments. To call the int method, we must explicitly enter

$\operatorname{disp}(\operatorname{add}(\operatorname{int} 32(\operatorname{val} 1), \operatorname{int32(\operatorname {val}2)))}$

MATLAB also performs automatic conversions based on array dimensions. If the MATLAB variable is a scalar (dimensions 1 by 1 ), it will be converted 
to a scalar value in Java. If the MATLAB variable has a one nonsingleton dimension (e.g., it is $\mathrm{N} \times 1$ or $1 \times \mathrm{N}$ ), it will be mapped to a 1-D Java array. If it has two nonsingleton dimensions, it will be mapped to a 2-D Java array. For example,

$\mathrm{z}=\left[\begin{array}{lll}1.1 & 1.3 ; 2.4 & 3.5\end{array}\right] ;$

will map to a Java double [] [ ] array.

Arrays of nonsimple data types are handled using MATLAB's javaArray function.

\subsubsection{The MATLAB disp command}

When a Java object is called by the MATLAB disp command, MATLAB will call the Java class's tostring method, which provides customized output depending on the definition within each Java class. Throughout the EASEE environ and geo packages (and most others packages in EASEE), an effort has been made to provide tostring methods that usefully summarize the properties of the object. What this means, in practice, is that disp can be used to display helpful information about the object. For example, typing

atm = HumidAir $(18.1,0.012) ;$

creates an instance of the HumidAir class (which will be discussed layer). When this object is displayed by typing

$\operatorname{disp}(\mathrm{atm})$

we see the following information:

Humid air: temperature 18.1 (C), specific humidity 0.012, pressure 101.33 (kPa)

\subsubsection{Java enumerations and inner classes}

Many classes in EASEE involve a programming pattern in which a Java enumeration class is used to define a number of types of an object. For example, the Helicopter class defines types for Apache, Blackhawk, and other helicopters. The soil class defines types for sandy soil, clayey soil, 
etc. While these enumerations are very useful and convenient when programming in Java, unfortunately they can be a bit awkward to use from MATLAB, particularly when they are defined inside another class. Thus, we describe here how to handle the Java enumerations from MATLAB.

As an example of an enumeration that is implemented in its own separate class (as opposed to an inner class), let us first consider land cover definitions in EASEE. (The land cover definitions will be discussed more fully later in this report; here our focus is on describing the programming pattern.) One of the land cover systems implemented in EASEE is the National Land Cover Dataset (NLCD) 2001. This system consists of 20 different land cover types. The class in EASEE that implements these types is called NationalLandcoverDataset2001Types, where "Types" is generally added to the end of an enumeration name. NationalLandcoverDataset2001Types, in this case, is itself an enumeration; that is, we do not have to deal with the complication of an inner class.

To import NationalLandcoverDataset2001Types, we type

import mil.army.usace.knee.environ. NationalLandcoverDataset2001Types;

The values method is used to create an array holding all of the available enumerations in the order they are defined:

NLCDTypes = NationalLandcoverDataset2001Types.values ();

If one were to simply type disp (NLCDTypes), MATLAB would provide a rather uninformative listing of references to Java objects. To actually list the land cover enumerations, one can loop through the array while calling the name method, as follows:

for $m=1$ : length (NLCDTypes), disp (NLCDTypes (m).name); end

This command will yield the following output:

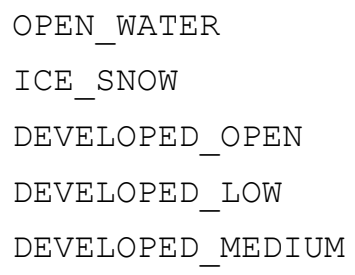




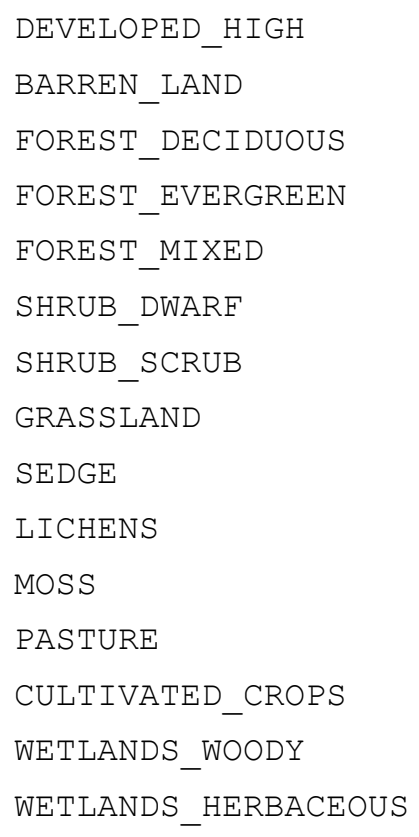

The previous listing shows the actual names of the enumerations as they would appear in the Java code. More useful, descriptive names can be listed using the tostring method in place of the name method. For example, 
Moss (74)

Pasture/Hay (81)

Cultivated Crops (82)

Woody Wetlands (90)

Emergent Herbaceous Wetlands (95)

The one-to-one correspondence of these descriptive names to the original enumerations should be clear. The values in parentheses correspond to an integer coding system for the land cover, which will be discussed later. Since the MATLAB disp command automatically invokes the tostring method, the following code would produce the same descriptive listing:

for $m=1$ : length (NLCDTypes), disp (NLCDTypes (m)); end

If we wish to create a new variable in MATLAB corresponding to one of these enumerations, for example, FOREST_DECIDUOUS, we can enter

lcCode = NationalLandcoverDataset2001Types.FOREST_DECIDUOUS;

Alternatively, we could count down the listing and note that FOREST DECIDUOUS is the eighth element of the array. Thus, equivalent to the preceding, we could use

lcCode $=$ NLCDTypes $(8)$;

Programming with the Java enumerations becomes a bit more cumbersome when the enumeration is implemented as a Java nested class. An example is accessing the predefined types in the HumidAir class, which represent the properties of humid air (a mixture of dry air and water vapor). A number of predefined characteristic air masses are defined within HumidAir by an inner class called Types. They can be listed by entering

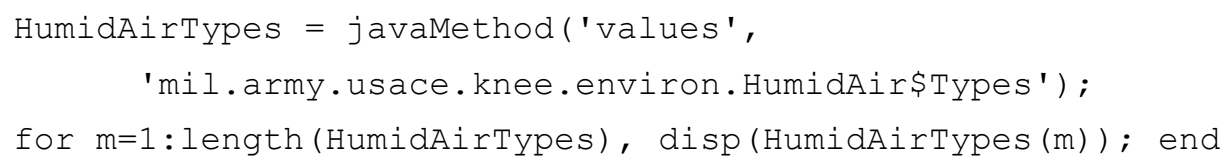

This will result in

Polar, winter

Polar, summer 


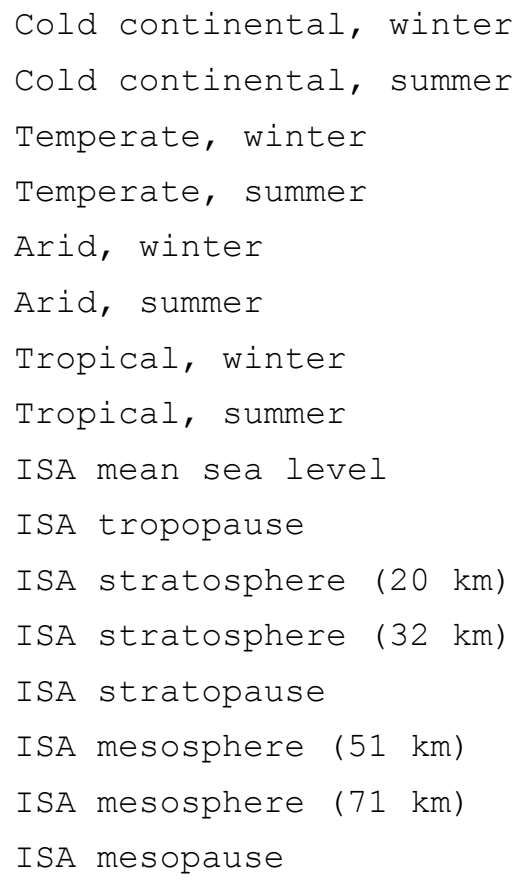

Note that the inner class is invoked from MATLAB using the $\$$ operator, which must be passed via the javamethod function. Therefore the Types in the HumidAir class cannot be accessed directly; the approach of creating an array to access the enumerations, as described above with respect to the land cover, must be used. Thus to create a HumidAir object with properties characteristic of temperate summer air, we would thus enter

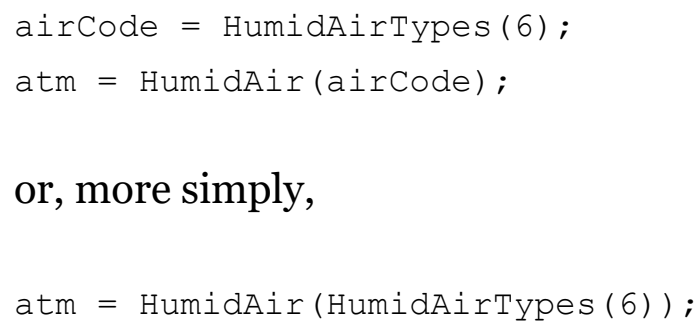




\section{Geographic Coordinates, Grids, and Elevation Maps}

In this section, we consider some features of EASEE that, while they do not pertain directly to acoustical calculations, do support those calculations in important ways. Specifically, we describe KNEE's geo package, which provides capabilities for setting and manipulating Earth coordinates and for representing data on geographic grids. To use the geo package, first import it by entering

import mil.army.usace.knee.geo.* ;

\subsection{Geographic coordinates}

The geo package supports Earth coordinate specifications in latitude/longitude, Universal Transverse Mercator (UTM), and Military Grid Reference System (MGRS). The latitude/longitude coordinates are generally specified in decimal degrees, whereas UTM and MGRS are specified in meters. The WGS84 Earth ellipsoid (datum) is used by default for all coordinate systems.

The Geocoord class in geo is used to create a geographic coordinate. Here are the primary four constructors for this purpose:

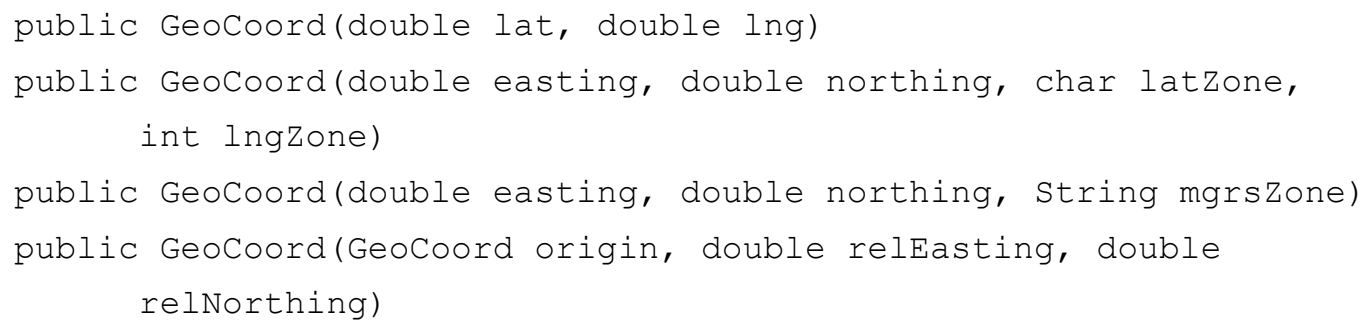

The first three of these correspond to the three coordinate systems mentioned above. The fourth specifies a coordinate relative to an origin coordinate but offset by indicated distances to the east and north of the origin coordinate. The offsets are interpreted based on the UTM projection in the grid zone of the origin.

For example, to create a coordinate at a latitude of $43.5^{\circ}$ and a longitude of $-72.4^{\circ}$ (i.e., $72.4^{\circ} \mathrm{W}$ ), we would enter 
$\operatorname{gc1}=\operatorname{GeoCoord}(43.5,-72.4) ;$

Once a coordinate has been constructed, we can use the following methods to retrieve its properties:

- getLat for the latitude (decimal degrees)

- getLng for the longitude (decimal degrees)

- getUTMEasting for the UTM easting (m)

- getUTMNorthing for the UTM northing (m)

- getLatzone for the UTM latitude zone (a character)

- getLngzone for the longitude zone number (an integer)

- getmarseasting for the MGRS easting (m)

- getMGRSNorthing for the MGRS northing (m)

- getmarszone for the MGRS grid zone (a string)

Furthermore, the following methods convert values to different units:

- tostring or tostringDecDeg (which are equivalent) displays the coordinate in latitude/longitude in decimal degrees.

- tostringDMS displays the coordinate in degrees/minutes/seconds.

- tostringUTM displays the coordinate in the UTM system.

- tostringMgRS displays the coordinate in the MGRS system.

Some other useful methods in the Geocoord class calculate quantities of one geographic coordinate relative to another. For each of these methods, the current instance of the geographic coordinate is the origin, whereas the specified argument is the termination. For example,

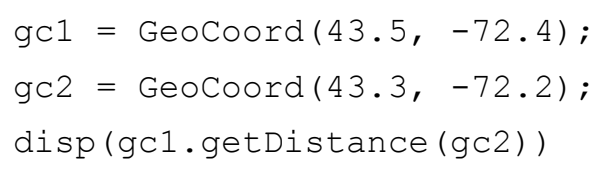

displays the distance between the geographic coordinates gc1 and gc 2 . The calculation is based on the UTM projection in the grid zone of gc1. The output in this case is $27.47 \mathrm{~km}$. The method getRelBearing returns the relative bearing (azimuth) of the termination relative to the origin. Hence, 
returns $142.04^{\circ}$, where the orientation is specified in the geographic convention, namely where $\mathrm{o}^{\circ}$ is northward and the angle increases clockwise. Therefore, in this case, gc2 is to the southeast of gc1. Similarly, getRelNorthing and getRelEasting return the relative distance to the north and relative distance to the east (as based on the UTM projected coordinates).

In addition to GeoCoord, there is a class called GeoCoord3D, which is very similar to Geocoord except that the constructors take an additional argument that indicates the height above ground level in meters.

\subsection{Geographic grids}

The geo package also enables the creation of geographic grids, by which is meant a regular (Cartesian) grid of geographic coordinates. The coordinates can be specified using latitude and longitude, UTM, or MGRS easting and northing. Note that a regularly spaced grid in latitude and longitude does not equate to a regularly spaced grid in distance. A 2-D grid can be created by specifying the coordinates of the southwest and northeast corners and the number of points in each direction:

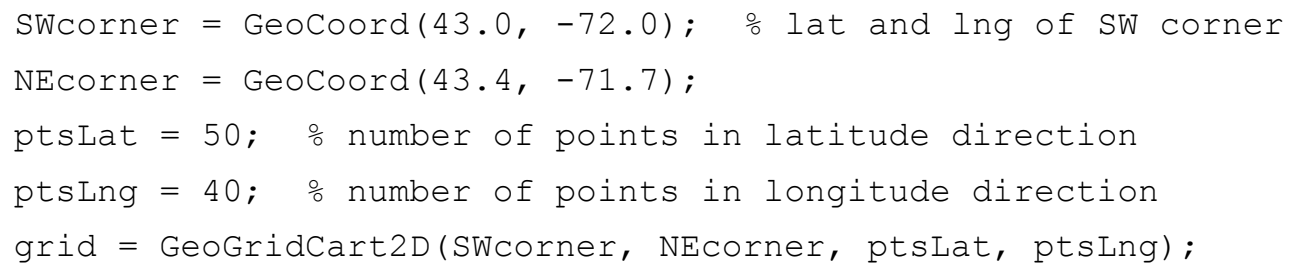

Note that the previous GeoGridCart2D constructor defaults to a latitude/longitude grid. It is immaterial that the corner coordinates were specified in latitude/longitude; they can be specified in any system. The following constructor is equivalent to the previous although the grid system and datum (Earth ellipsoid model) are specified explicitly:

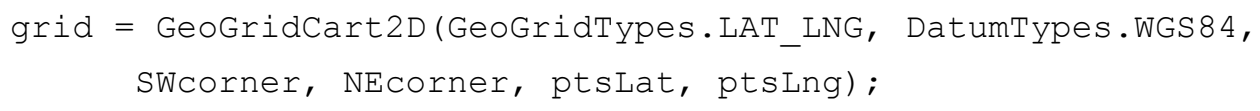

Alternatively, if we desire a UTM grid, we may specify:

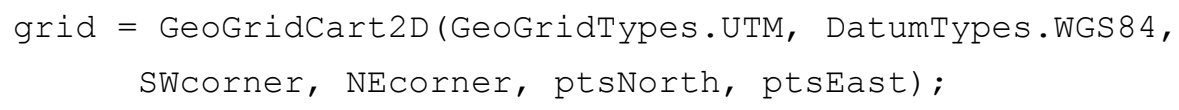


where ptsNorth and ptsEast are the number of points to the north and to the east, respectively. The grid may also be specified by indicating the spacing between grid points in each direction:

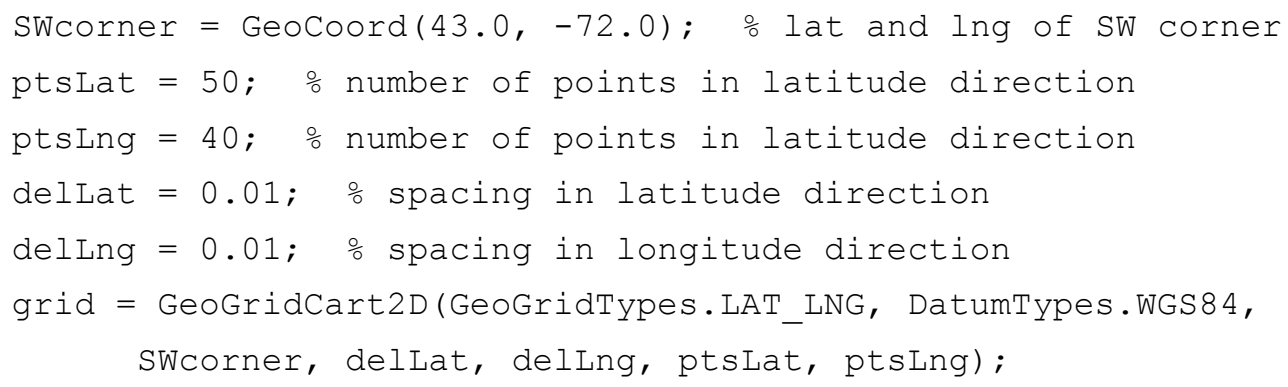

Within Java, the grid data are, by convention, stored such that latitude (or northing) corresponds to the inner, or faster, varying array index, whereas longitude (or easting) corresponds to the outer, or slower, varying grid index. That is, for a 2-D array $f$, use $f[i][j]$ to retrieve a point at longitude index $i$ and latitude index $j$.

There are two extensions of GeoGridCart2D, namely GeoGridCart2DInt and GeoGridCart2DDouble. These include grids to hold 2-D integer array data (Java int [ ] [ ] ) and 2-D double array data (Java doub le [ ] [ ]), respectively. For example, one might store a digital elevation model as a GeoGridCart2DDouble (as will be discussed momentarily). GeoGridCart2DInt includes methods called setDataGrid and getDataGrid2DInt, which set and retrieve the integer data array. Similarly, GeoGridCart2DDouble includes methods called setDataGrid and getDataGrid2D, which set and retrieve the double data array.

The geo package also includes a class called GeoGridcart3D, which accommodates a three-dimensional (3-D) grid. The third coordinate is altitude.

\subsection{Digital elevation, surface, land cover, and soil grids}

A digital elevation model (DEM) represents the terrain elevation as a function of the geographic coordinates. Normally, the DEM is understood to represent the "bare Earth" elevation (i.e., the elevation when all the objects on the surface, such as vegetation and buildings, are removed). The digital surface model (DSM) represents the elevation of the bare Earth plus the surface elements. Currently, the acoustical calculations in EASEE use only the DEM; the surface elements are ignored. 
Typically, the DEM and DSM would be read from a file, but to illustrate the concept here for how a DEM is constructed and used in an EASEE calculation, we contrive a DEM using the MATLAB peaks function for the terrain elevations:

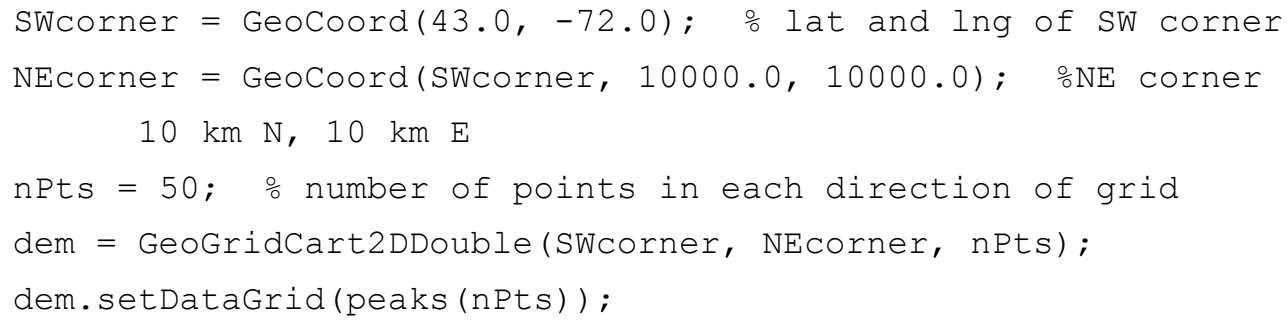

This code snippet creates a domain with the southwest corner at the specified latitude and longitude and with the northeast corner $10 \mathrm{~km}$ to the north and $10 \mathrm{~km}$ to the east of the southwest corner, as based on a UTM coordinate projection. A geographic grid of size of $50 \times 50$ points is then created, and the values of that grid are set using the peaks function.

Geographic grids are also used to represent land cover and soil properties, which vary along the Earth-air interface. Although we have not yet discussed in detail how land cover and soil types are represented (that will be the topic of sections 5.2.3 and 5.2.4, respectively), for present purposes it is sufficient to understand that these properties are represented by integers, which encode the type of land cover or soil according to standardized systems. The standardized system is described by a "decoder" class in EASEE. Specifically, suppose lcDecoder and soil Decoder are objects specifying the mapping of integer data to land cover and soil types, respectively, and that lcGrid and soilgrid contain the actual integer data grids for the land cover and soil. Normally, these land cover or soil grids would be read from a file, such as a GeoTiff format file. However, for illustrative purposes, it is also worthwhile to consider how such data could be specified manually, as we did with the DEM. Building on the previous example where we used the MATLAB peaks function to construct a DEM, let us suppose that the land cover type consists of woody wetlands at negative elevations and mixed forest at positive elevations. Referring to the list of NLCD 2001 land cover types in section 3.4.6, we see that the codes for these land cover types (shown in the parentheses) are 90 and 43, respectively. Hence, we set 


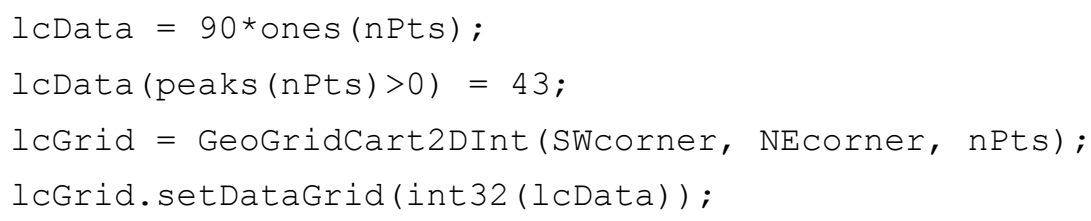

Here, the MATLAB int32 function converts the double array to a 32-bit integer array, which corresponds to the data type int [ ] [ ] in Java. Similarly, we can construct a soil grid consisting of organic silts (FASST [Fast AllSeason Strength] soil type number 11 [see section 5.2.4 for information about FASST]) at negative altitudes and silty clayey sand (FASST soil type number 16) at positive altitudes:

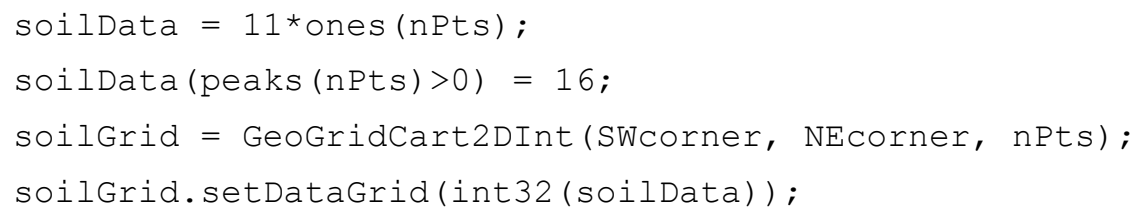




\section{Environmental Representations}

In this section, we discuss EASEE's environ package, which represents environmental data. By environment, we mean the atmosphere, Earth surface characteristics (terrain), and solid-earth (subsurface) properties. In this section, we show how to create (instantiate, in Java terminology) objects representing the environment, for the eventual purpose of using them in acoustical calculations. EASEE supports a great variety of approaches to creating the environmental objects. Information on approaches not explicitly described here can be obtained by examining the original Java code or Javadoc.

From a software-design perspective, EASEE has its own, complete internal representation of the atmosphere, surface, and subsurface, which serves as a data abstraction layer between the various types of external environmental models and data and the signal and sensor models found in EASEE. The main components of this representation are the atmospheric profiles, atmospheric surface layer (part of the atmosphere adjacent to the ground), subsurface profiles (primarily intended for seismic calculations), terrain elevations, land cover properties, soil type, soil moisture, snow type, and snow depth. The value of such an abstraction layer is that it isolates the signal and sensor models from the external environmental models and data; when a new environmental model or data source is interfaced with EASEE, all of the signal and sensor models will continue to function without modification.

EASEE currently has three basic environmental representations. All three are subclasses of the abstract class Environscenario. Figure 3 shows the hierarchy of environmental representations and the data elements at each stage in the hierarchy. In order of increasing complexity, the three subclasses are homogeneous (the EnvironHomo class), 1-D (the EnvironvertProf class), and 3-D (the EnvironThreeDim class). Here, "homogenous" means that the atmosphere and subsurface have constant properties. Terrain properties, such as elevation and land cover, may nonetheless vary along the Earth surface. The 1-D representation includes atmospheric and subsurface profiles that vary vertically. The 3-D representation includes both vertical and horizontal variability. At the time of the writing of this report, the 3-D representation was nearly com- 
plete although modeling capabilities using the 3-D atmospheric and subsurface fields had yet to be implemented. Thus, we do not describe the EnvironThreeDim class here.

Figure 3. Hierarchy of EASEE's environmental classes. Phrases in bold are the main environmental classes within EASEE, and italicized phrases are subclasses of the foremost environmental class-EnvironScenario. All other listings are fields (e.g., friction velocity is a field within AtmosSfcLayer, which is a field within AtmosOneDim, which is a field within EnvironVertProf). All phrases with only capitalized terms (e.g., EnvironVertProf) are instances of classes. Blue font color indicates recent additions.

EnvironScenario (common to all representations)

Digital elevation map

Digital surface map

Land cover type grid

Soil type grid

Snow type grid

Snow depth grid

Soil moisture grid

$>$ EnvironHomo (homogeneous atmosphere/ground)

Homogeneous air layer

Homogeneous solid layer

EnvironVertProf (horizontally stratified environment)

AtmosOneDim (1-D atmosphere)

AtmosSurflayer

Friction velocity, sensible/latent heat fluxes, surface temperature, etc. AtmosVertProf

Profiles of wind, temperature, humidity, and pressure Clouds

Low-, mid-, and high-cloud layers, each with height and cover fraction SubSurfOneDim (1-D subsurface)

SeismicVertProf

Profiles for density, compressional and shear wave speed, attenuation

$>$ EnvironThreeDim (3-D environment)

AtmosThreeDim (3-D atmosphere)

2-D grid of atmospheric profile locations

AtmosSurfLayer[][] (2-D array of AtmosSurfLayer)

AtmosVertProf[][] (2-D array of AtmosVertProf)

Clouds[]]] (2-D array of Clouds)

SubSurfThreeDim (3-D subsurface)

2-D grid of subsurface profile locations

Seismic VertProf[][] (2-D array of SeismicVertProf)

In this section, we describe construction of the EnvironHomo and EnvironVertProf objects. Before actually getting to that point, we discuss construction of a number of objects of many types that are components of the environmental classes (e.g., air and solid representations). 
For the examples in this section, KNEE's environ and geo packages must both be imported using the commands (typed at the MATLAB prompt):

import mil.army.usace.knee.environ. *;

import mil.army.usace.knee.geo. * ;

\subsection{Atmospheric constants and conversions}

As a preliminary, we describe in this subsection a simple, but useful, capability in EASEE, namely the availability of a number of constants related to significant physical properties, particularly the thermodynamic properties of air and water. These are found in the Atmosconstants class in KNEE's environ package. To display gravitational acceleration, for example, enter

disp (AtmosConstants.GRAV_ACC)

To display standard sea-level pressure, enter

disp (Atmosconstants.SEA_LEVEL_PRESS)

Or, to display the freezing point in Kelvin, enter

disp (AtmosConstants.FREEZING_PT_KELVIN)

Many other properties are available, which can be seen by examining the Javadoc or the Java code for the Atmosconstants class. Alternatively, one may list the names of each physical property within Atmosconstants by typing the following:

fieldnames (AtmosConstants)

This produces

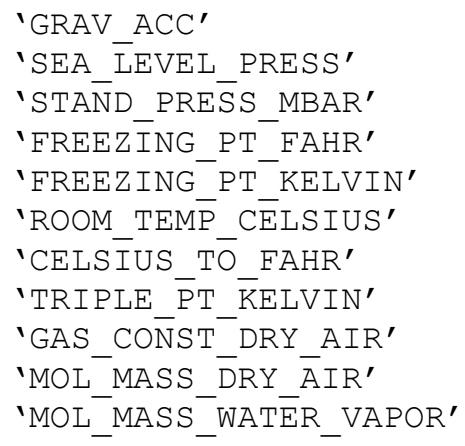


'MOL MASS RATIO'

'SPEC

'SPEC HEAT DRY AIR'

'SPEC_HEAT_WATER_VAPOR'

'LATEN̄T_HEĀT_WATER_VAPOR'

'POISSON CON $\bar{S} T$ '

' DRY_LAPSE_RATE'

The AtmosConstants class also provides static methods for converting temperature and pressure between various units. Either scalar values or arrays may be passed to these methods. The method degCToDegF, for example, converts degrees Celsius to degrees Fahrenheit. Thus

disp (AtmosConstants.degCToDegF ( 0.0 100.0 $])$ )

displays an array with values of 32.0 and 212.0. Similarly,

- degCTоDegk converts temperature from degrees Celsius to Kelvin,

- degFToDegC converts temperature from degrees Fahrenheit to degrees

Celsius, and

- degKToDegc converts temperature from Kelvin to degrees Celsius.

- paTombar converts pressure from Pascals to millibars,

- mbartopa converts pressure in the other direction,

- paToAtm converts pressure from Pascals to atmospheres, and

- atmToPa converts pressure in the other direction.

The AtmosConstants class also has static methods for converting between ordinary temperature and potential temperature:

- tempKToPotTempK converts from ordinary to potential temperature.

- potTempKтотеmpK converts in the other direction.

These methods each take two arguments, the first being the temperature in Kelvin, the second being the pressure in Pascal.

\subsection{Environmental components}

In this subsection, we describe the most important components of the EASEE environmental representations: the EnvironHomo and EnvironVertProf classes. 


\subsubsection{Humid air representation}

The HumidAir class represents a mixture of dry air and water vapor. Naturally, it plays a particularly important role in modeling the atmosphere and its impact on sound propagation. A HumidAir object is constructed by specifying temperature, humidity, and pressure. The most basic constructor is

HumidAir(double temp, double specHum, double press)

where temp is the temperature in degrees Celsius, specHum is the specific humidity (ratio of the mass of water vapor $[\mathrm{kg}]$ to the total mass of the air parcel $[\mathrm{kg}]$ ), and press is the pressure in Pascals. For example, entering

atm = HumidAir $(18.1,0.012,99000.0)$;

creates a MATLAB variable called atm, which holds a Java HumidA ir object representing air at a temperature of $18.1^{\circ} \mathrm{C}$, specific humidity of 0.012 , and pressure of 99000.0 Pa.

The constructor may also be called with no arguments, a single argument (temperature), or two arguments (temperature and specific humidity). In these cases, default values are used for the unspecified variables. The default values are a temperature of $20^{\circ} \mathrm{C}$, zero humidity, and standard sealevel pressure (101325 Pa). For example,

atm = HumidAir $(18.1,0.012)$;

would assume standard sea-level pressure.

If we wish to specify the humidity in some way other than specific humidity, we can use HumidAir's conversion capabilities. Conversions are specified using the following integer codes: $1=$ relative humidity (\%), $2=$ molar concentration, $3=$ specific humidity, $4=$ mixing ratio, and $5=$ dew-point temperature $\left({ }^{\circ} \mathrm{C}\right)$. The code precedes the humidity value. Thus, if the input value is a relative humidity of $40 \%$, the corresponding constructor (assuming sea-level pressure) would be

atm = HumidAir $(18.1$, int32(1), 40.0); 
A number of predefined characteristic air masses are also available. These are defined by a nested class in HumidAir called Types, which can be used to construct a HumidAir object, as described in section 3.4.6.

The following are some useful getter methods for the HumidAir class:

- getTemp, which gets the temperature in degrees Celsius

- getTempKelvin, which gets the temperature in Kelvin

- getTempFahr, which gets the temperature in degrees Fahrenheit

- getPress, which gets the pressure in Pascals

- getPressmbar, which gets the pressure in millibars

- getPressAtm, which gets the pressure in atmospheres

- getDensity, which gets the density in kilograms per cubic meter

- getSpecHum, which gets the specific humidity

- getRelHum, which gets the relative humidity in percent (\%)

- getDewPoint, which gets the dew-point temperature in degrees Celsius

- getmixRat, which gets the mixing ratio

- getMolHum, which gets the molar humidity

For example, the density can be displayed by entering

disp (atm.getDensity)

The HumidAir class also contains a static method called convHum, which converts between different measures of humidity. The humidity measure is specified using the previously described integer codes. Thus, to convert from a relative humidity of $52 \%$ to mixing ratio, for example, we would enter

disp (HumidAir.convHum (1, 4, 52.0))

The preceding assumes a temperature of $20.0^{\circ} \mathrm{C}$ at sea-level pressure. To explicitly specify the temperature and pressure, add them as additional arguments. For a temperature of $3.0^{\circ} \mathrm{C}$ and pressure of $10^{5} \mathrm{~Pa}$, enter

disp (HumidAir.convHum (1, 4, 52.0, 3.0, 10^5))

\subsubsection{Solid-earth representation}

The Solidisolinear class represents the properties of isotropic, linear (primarily solid) materials. This SolidIsoLinear class has an associated 
class called SolidisoLinearTypes, which defines several common solid materials of interest. The types available can be listed by entering

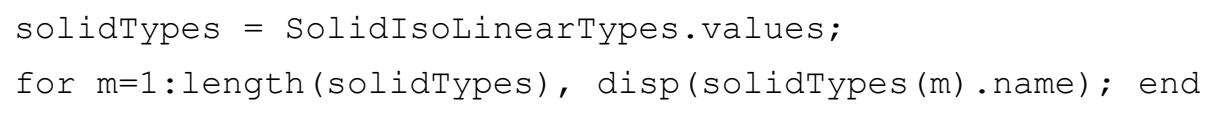

Omitting the name method above produces the following descriptive list:

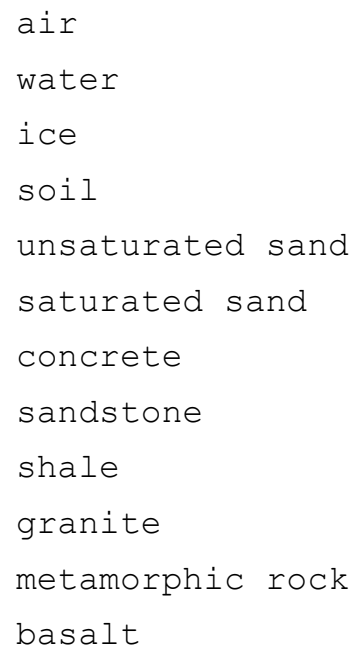

To construct a SolidisoLinear object, we can simply pass the desired SolidIsolinearTypes enumeration (e.g., for unsaturated sand)

solid = SolidIsolinear (solidTypes (5)); 
or, more directly,

solid = SolidIsoLinear(SolidIsoLinearTypes.SAND_UNSAT);

Similarly, if we wish to construct a subsurface of granite (the tenth element in the array), we would enter

subsurf = SolidIsoLinear (solidTypes (10));

or

solid = SolidIsoLinear(SolidIsoLinearTypes.GRANITE);

Alternatively, if we do not wish to use one of the predefined types, the solid properties can be specified directly using one of the constructors

Solidisolinear(double rho, double K, double G)

or

Solidisolinear(double rho, double cp, double cs, double $Q p$, double Qs)

The first constructor specifies the density, bulk modulus, and shear modulus and assumes the medium is nonattenuative. The second constructor specifies the density, compressional (P-) wave speed, shear (S-) wave speed, quality factor for compressional waves, and quality factor for shear waves. The quality factors characterize the wave attenuation and may be set to 1000 for a nonattenuative medium.

\subsubsection{Land cover representation}

The land cover is normally set using one of the predefined types, which are based on a number of standardized systems for enumerating land cover. The systems currently supported by EASEE include NLCD 1992 and 2001 (Java classes NationalLandcoverDataset1992Types and NationalLandcoverDataset2001Types, respectively), GeoCover (GeoCoverLCTypes), WorldView (WorldView2LCTypes), and VisNav (VisnavLCTypes). Earlier, we discussed how to list the land cover enumerations associated with NationalLandcoverDataset2001Types. The same 


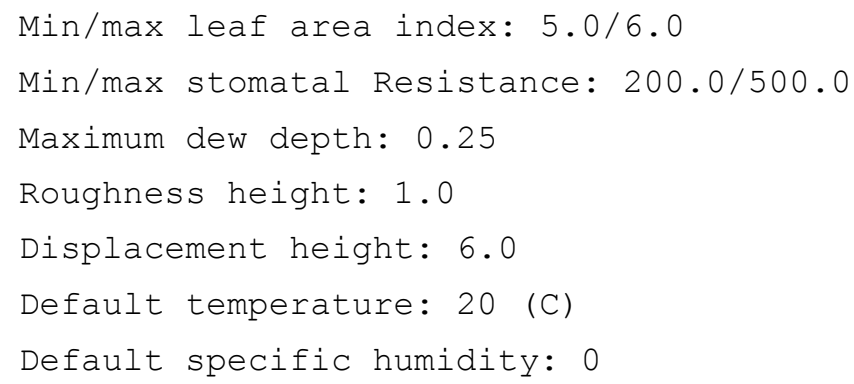

In the listings of the land cover types, the values in parentheses correspond to integer codes, which represent the land cover type in data files; for example, a code of 2 in the GeoCover system is "Forest, Evergreen," whereas 6 would correspond to "Urban/Built-Up." The integer code can be displayed using the getLcCode method:

disp (lcCode.getLcCode)

\subsubsection{Soil representation}

The soil properties are set in much the same manner as the land cover. For soil types, EASEE currently supports only one system, namely the Fast AllSeason Strength (FASST) soil types, which are an extension of the U.S. Geological Survey soil classes (Frankenstein and Koenig 2004). These are enumerated in the class FASSTSOIlTypes. We can list the predefined soil types by entering

FASSTTypes = FASSTSoiltypes.values();

for $m=1$ : length (FASSTTypes), disp (FASSTTypes (m)); end

This results in the following output:

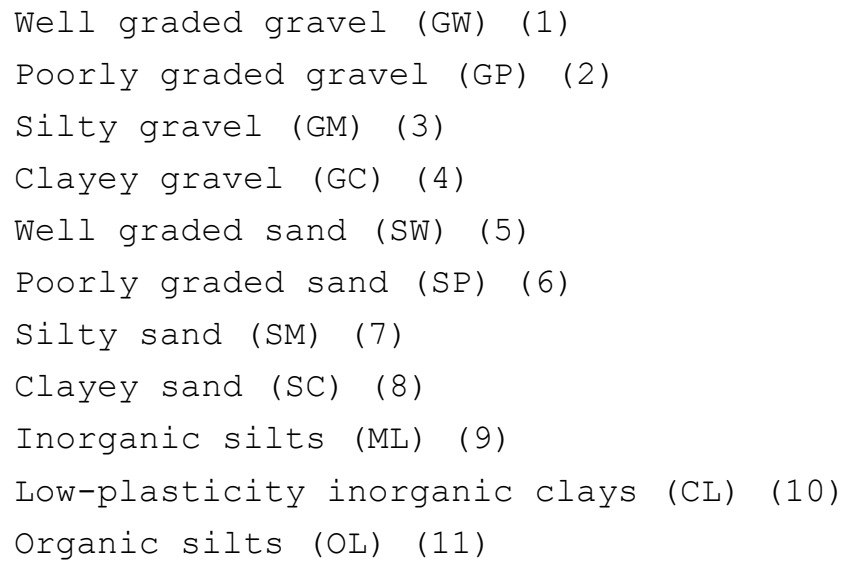




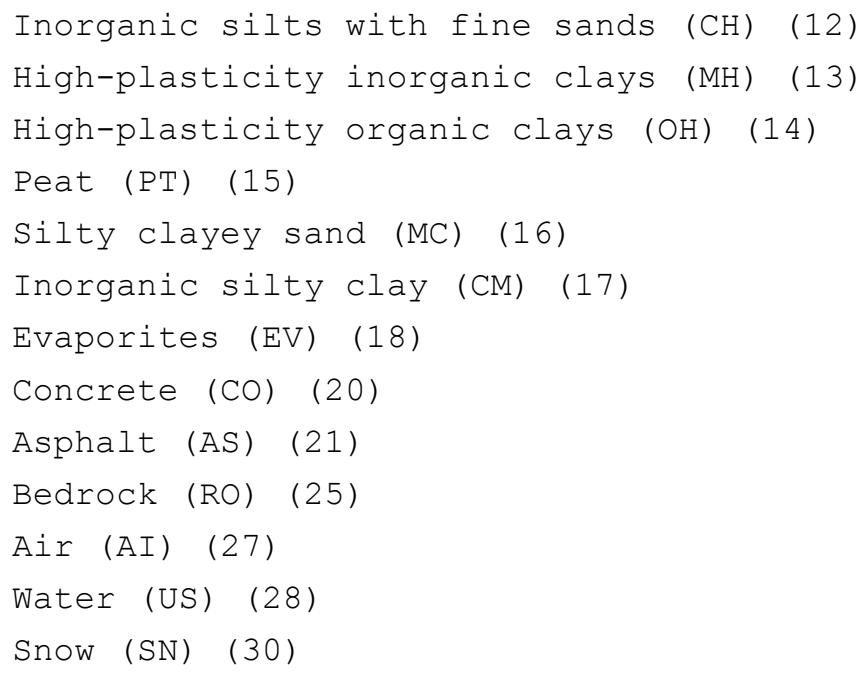

A soil object is constructed by passing the desired enumeration. Thus, if we wish to set the soil type to silty clayey sand, which is the sixteenth element in the preceding array, we would enter

soil = Soil (FASSTTypes (16));

where soil (lowercase) is now an instance of EASEE's soil (initial cap) Java class.

As with the land cover enumerations, the values in parentheses in the previous listing correspond to the integer codes, which represent the soil type.

\subsubsection{Snow representation}

The SnowTypes class represents five different characteristic types of snow, based on data provided by Dr. Don Albert of the ERDC Cold Regions Research and Engineering Laboratory (pers. comm.). To list these, enter

snowTypes $=$ SnowTypes.values ();

for $\mathrm{m}=1$ : length (snowtypes), disp(snowTypes (m)); end

The result is

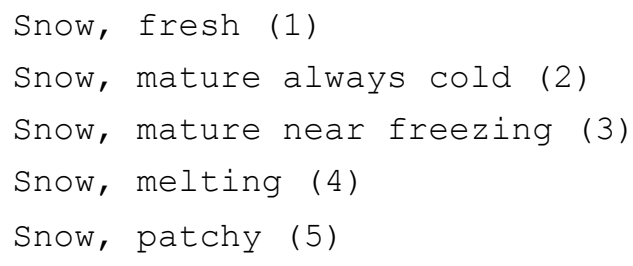


Snow is actually regarded by EASEE as a class of soil types; that is, the snow layer is, in effect, a type of soil on top of the normal soil layer. Thus, to create a snow object, pass the desired enumeration to the soil constructor. For example, to designate melting snow,

snow $=$ Soil (snowTypes (4));

\subsubsection{Atmospheric surface layer}

The atmospheric surface layer (ASL) is defined as the part of the atmosphere in which the fluxes (momentum, heat, and moisture) are nearly equal to their values at the surface (Stull 1988). Typically, the ASL extends from the Earth's surface to about 50-200 m above. The environmental layer in EASEE includes a representation of the ASL, namely the Atmossurftayer class.

The following Atmossurftayer constructor specifies the most important surface-layer parameters directly:

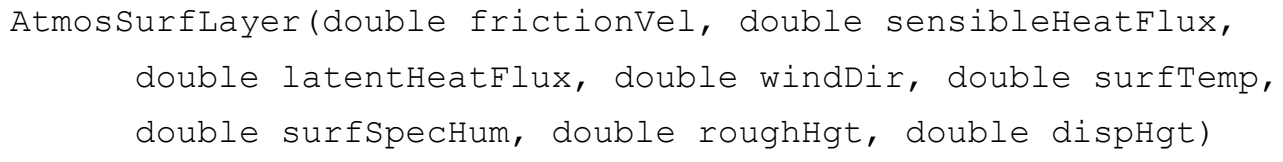

Here,

- frictionvel is the friction velocity,

- sensibleHeatFlux is the sensible heat flux,

- latentHeatFlux is the latent heat flux,

- windDir is the wind direction in radians (in the Cartesian convention, where $\mathrm{O}$ is to the east and $\pi / 2$ is to the north),

- surftemp is the "surface" temperature, and

- surfSpecHum is the "surface" specific humidity.

We included the quotes around "surface" because what is actually meant here is the temperature and humidity at $2 \mathrm{~m}$ height, which is the standard meteorological height for surface temperature and humidity observations. Although the roughness and displacement heights (roughHgt and dispHgt) are not part of the surface-layer representation per se, they are needed internally by the constructor to deduce the wind speed at the standard wind observation height, $10 \mathrm{~m}$. Finally, the Atmossurftayer class includes static 
methods that convert the above Cartesian wind direction to the more familiar meteorological convention $\left(\mathrm{O}^{\circ}\right.$ from the north, increasing clockwise-convToMetConv) and vice versa (convToCartConv).

An alternative approach to specifying the surface layer involves the constructor

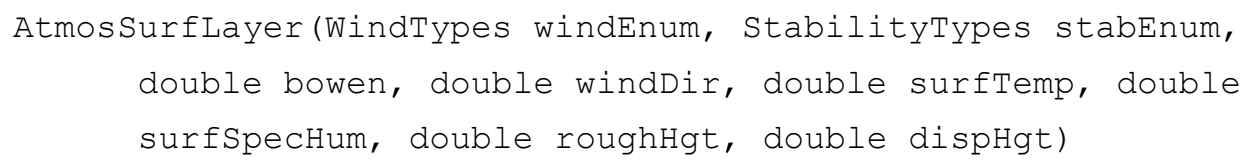

This constructor is similar to the previous, except that the first three arguments have been replaced by an enumerator for the wind category (windenum), an enumerator for the stability category (stabEnum), and the value of the Bowen ratio. The Bowen ratio is the ratio of the sensible to the latent heat flux at the surface, which is often assumed to be a constant for a particular land cover type. Alternatively, we could use the following constructor:

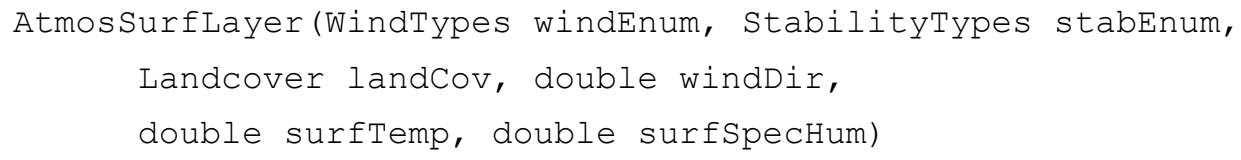

in which case the Bowen ratio, roughness height, and displacement height are automatically set to default values based on the land cover type.

To set a value for windEnum, first list the various wind categories as follows:

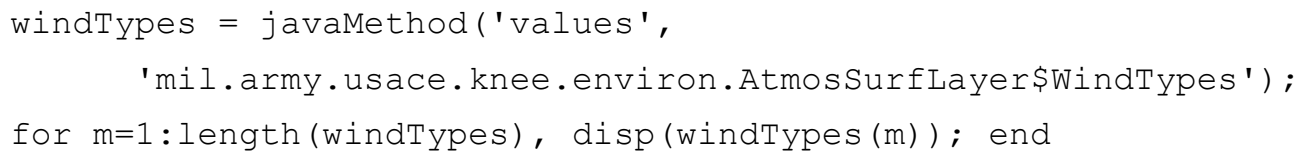

This will display the available categories:

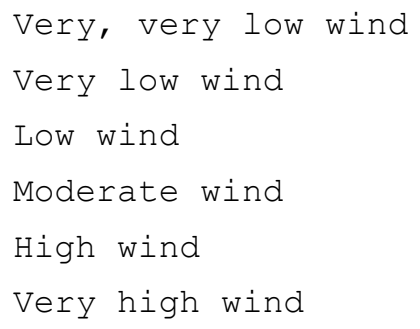


Thus we see that windTypes (4) would correspond to the moderate wind category, etc. For the stability categories,

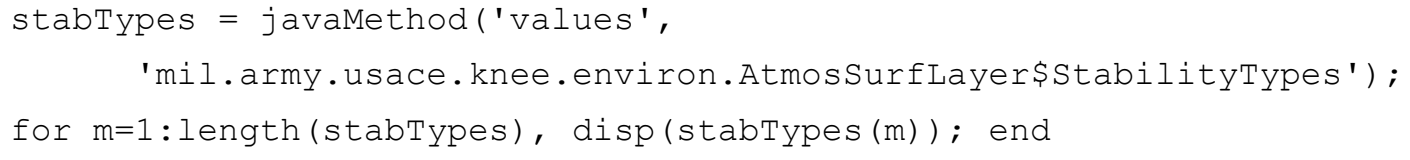

Thus, to construct a surface layer with moderate wind and very unstable stratification, with a grassy ground surface, wind blowing to the east, a surface temperature of $20^{\circ} \mathrm{C}$, and surface specific humidity of 0.009 , we would set

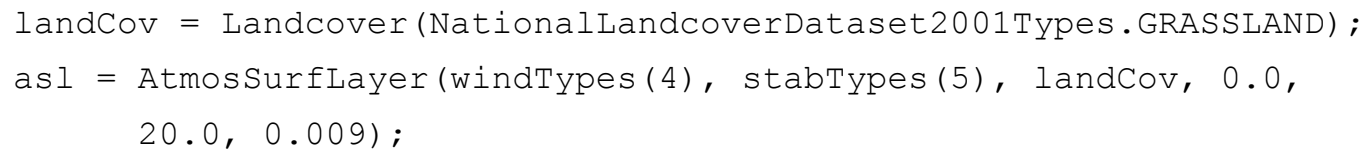

The AtmosSurfLayer class has a rich set of getter methods:

- getFrictionvel and getustar are equivalent and both return the friction velocity.

- getSensibleHeatFlux returns the sensible heat flux.

- getLatentHeatFlux returns the latent heat flux.

- getTstar returns the surface-layer temperature scale.

- getestar returns the surface-layer humidity scale.

- getBowenRatio returns the Bowen ratio.

- getWindDirCart returns the wind direction in the Cartesian convention (radians).

- getWindDirmet returns the wind direction in the meteorological convention (degrees).

- getSurfWindSpeed returns the surface wind speed.

- getobsHgtwind returns the observation height for the surface wind. 
- getSurftemp returns the surface temperature.

- getobshgtTemp returns the observation height for the surface temperature.

- getSurfSpecHum returns the surface specific humidity.

- getSurfRelHum returns the surface relative humidity.

- getSurfDewpoint returns the surface dew-point temperature.

- getobshgtHum returns the observation height for the surface humidity.

- getobukhovLength returns the Obukhov length.

- getcv2 returns the structure-function parameter for velocity.

- getCт2 returns the structure-function parameter for temperature.

Methods are also available to calculate statistics related to turbulence; these can be found by typing methods ('Atmossurflayer') or by examining the Javadoc or the original code.

\subsubsection{Atmospheric vertical profiles}

Many constructors are available to specify the atmospheric profiles. The following constructor, which assumes a homogeneous, motionless atmosphere, is the simplest:

AtmosVertProf(double[] profHgt, HumidAir air)

Here, profHgt contains the heights in meters above sea level at which the profiles are to be specified, and air is a humid air object specifying the properties of the homogeneous atmosphere.

The following constructor is used to specify the full atmospheric profiles directly:

AtmosvertProf (double[] profHgt, double[] vx, double[] vy,
double[] T, double[] q, double[] P)

Here, $v x$ is the eastward component of the wind $(\mathrm{m} / \mathrm{s})$, vy is the northward component $(\mathrm{m} / \mathrm{s}), \mathrm{T}$ is the temperature $\left({ }^{\circ} \mathrm{C}\right)$, $\mathrm{q}$ is the specific humidity, and $\mathrm{P}$ is the ambient pressure $(\mathrm{Pa})$. All of the arrays passed to this constructor must be the same length as profigt.

In many applications, it can be useful to model the profiles using the Monin-Obukhov similarity theory (MOST). (See, for example, Stull 1988.) The following two constructors are available for this purpose: 
AtmosVertProf (double[] profHgt, AtmosSurflayer asl, double roughHgt, double dispHgt)

and

AtmosVertProf(double[] profHgt, AtmosSurflayer asl, Landcover landCov)

Here, as 1 is an atmospheric surface layer object as described in section 5.2.5. The first of these constructors specifies the roughness and displacement heights directly; the second infers them from a land cover specification. The following code illustrates the usage of this constructor, including the creation of a plot of the temperature, specific humidity, and wind speed profiles, as shown in Figure 4:

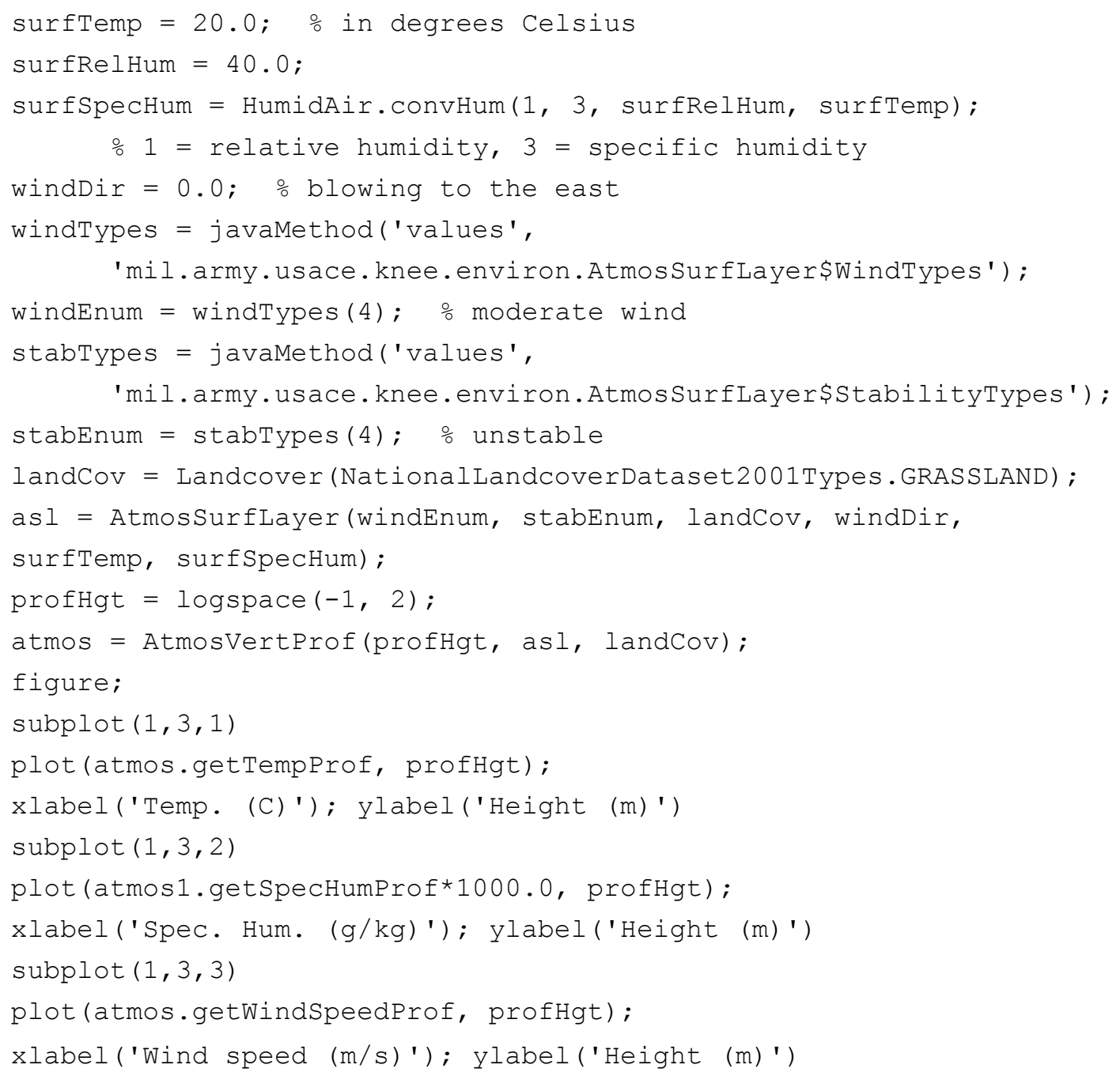


Figure 4. Vertical profiles of temperature, specific humidity, and wind speed as created by specification of atmospheric surface layer properties for a moderately windy, unstable case.
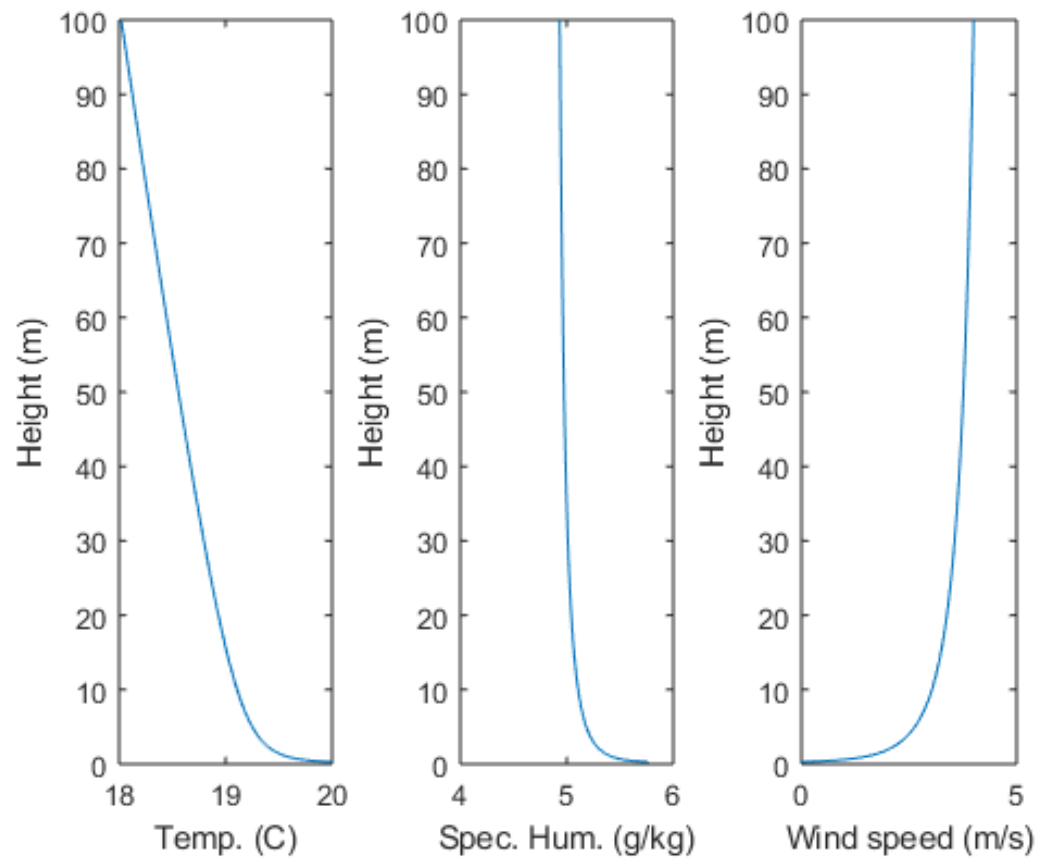

Profiles are also available for benchmark acoustical calculations. The constructor in this case is

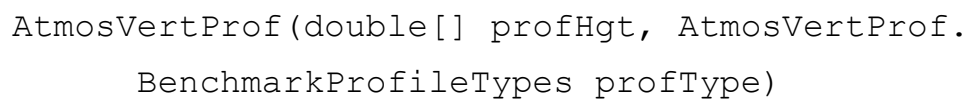

where proftype specifies the particular benchmark case. To list the available benchmark cases (which are a nested class of AtmosVertProf), enter

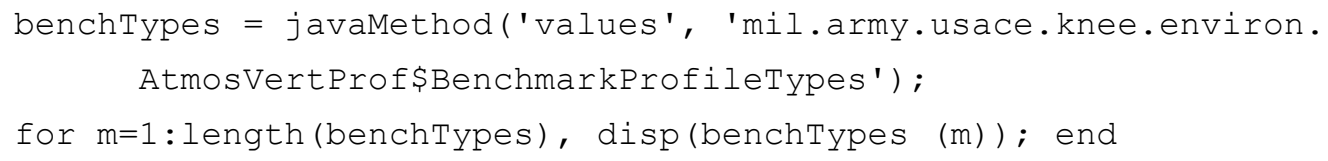

The following benchmark cases are then displayed:

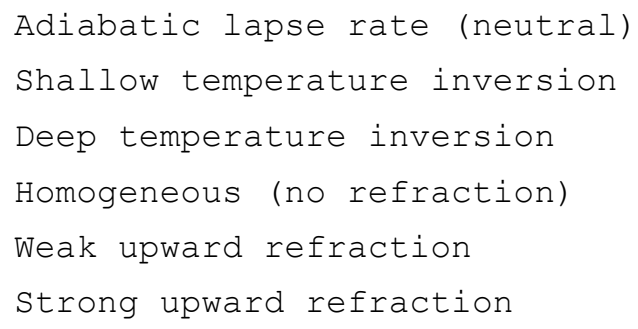


Downward refraction
Acoustic benchmark 1 (from Attenborough et al. 1995)
Acoustic benchmark 2 (from Attenborough et al. 1995)
Acoustic benchmark 3 (from Attenborough et al. 1995)
Acoustic benchmark 4 (from Attenborough et al. 1995)

If we want to construct the benchmark profiles for Case 2 from Attenborough et al. (1995) with the profile evaluated at $5 \mathrm{~m}$ increments up to an altitude of $100 \mathrm{~m}$, we would enter the following:

profHgt $=0.0: 5.0: 100.0 ;$

atmos = AtmosVertProf (profHgt, benchTypes (9));

Once the atmospheric profiles have been constructed, the AtmosvertProf class offers many convenient getter methods to retrieve their properties. Besides the profiles specified explicitly by the constructor, additional profiles can be determined based on the ideal gas law and other relationships:

- getProfHgt returns the heights at which the profiles are specified (in meters, starting at the lowermost point and increasing upward).

- getPressprof returns the pressure profile in Pascals.

- getPressprofmbar returns the pressure profile in millibars.

- getDensity returns the air density profile.

- getTempProf returns the temperature profile in degrees Celsius.

- getTempProffahr returns the temperature profile in degrees Fahrenheit.

- get InvHgt returns the height of the temperature inversion at the top of the boundary layer in meters.

- getSpecHumProf returns the specific humidity profile.

- getMixRatProf returns the water-vapor mixing-ratio profile.

- getRelHumProf returns the relative humidity profile.

- getwindSpeedProf returns the wind profile.

- getWindDirCartProf returns the wind direction in the Cartesian convention (radians).

- getWindDirMetProf returns the wind direction in the meteorological convention (degrees).

- getWindProfe returns the component of the wind velocity directed to the east.

- getWindProfN returns the component of the wind velocity directed to the north. 
All the methods with Prof in their names return double [] arrays with one element for each profile height.

\subsubsection{Clouds}

The clouds class defines cloud layers in the atmosphere. The most basic constructor has no arguments, namely

\section{Clouds ( )}

This constructor specifies clear skies (absence of cloud cover). The following is the next simplest constructor:

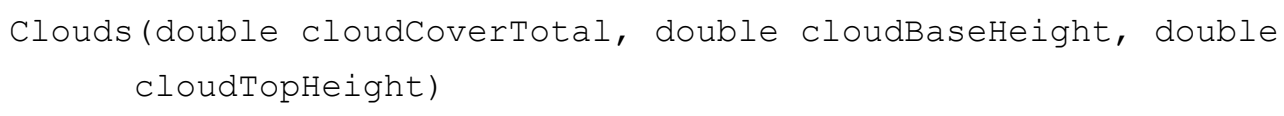

which specifies a single cloud layer with the indicated fractional coverage (cloudCoverTotal, a value between o and 1), cloud base height, and cloud top height. The constructor

Clouds(CloudLayer cloudstow, Cloudtayer cloudsMid, Cloudtayer cloudsHigh)

specifies three separate cloud layers at low, medium, and high altitudes. Each of these cloud layers is constructed using

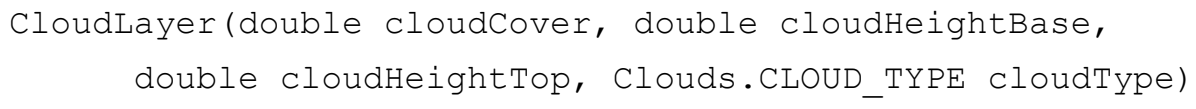

where the parameters are the fractional cloud coverage; the cloud base height; the cloud top height; and the type of cloud, which is a nested class within the clouds class.

Some of the useful getter methods in the clouds class include getcloudsLow, getCloudsMid, and getCloudsHigh, which retrieve the lower, middle, and upper cloud layers (cloudtayer objects), respectively. For the cloudtayer objects, the method getcloudCoverTotal gets the total cloud cover fraction, getcloudBaseHeight gets the cloud base height in meters, and getcloudTopHeight gets the cloud top height in meters. 


\subsubsection{Seismic vertical profiles}

The seismic vertical profiles are defined by the seismicvertProf class and contain information needed to model seismic wave propagation in the subsurface. The following constructor for SeismicVertProf specifies the profiles directly:

SeismicVertProf(double[] profDepth, double[] cp, double[] cs, double[] Qp, double[] Qs, double[] rho)

Here, profDepth is an array indicating the profile depths (meters below ground level); and the remaining arguments are arrays of the same length, which specify the material properties of the seismic layers. Specifically, $\mathrm{cp}$ is the compressional wave phase speed $(\mathrm{m} / \mathrm{s})$, cs is the shear wave phase speed $(\mathrm{m} / \mathrm{s}), Q p$ is the quality factor for compressional waves, $Q \mathrm{~s}$ is the quality factor for shear waves, and rho is the density $(\mathrm{kg} / \mathrm{m} 3)$. From these basic five profiles, other material profiles of interest in seismological calculations readily follow.

Alternatively, the profiles can be specified using layers of SolidIsoLinear objects (e.g., SOIL or GRANITE). The available constructors include

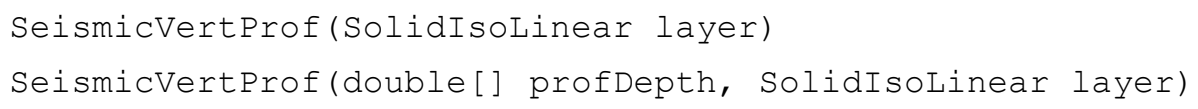

and

SeismicVertProf(double[] profDepth, SolidIsolinear[] layers, double[] transDepth)

The first two of these constructors specify a single solid type, which is used throughout the entire subsurface. In the first version, default profile depths are used, whereas in the second, they are specified explicitly. Although the seismic profiles will not vary as a function of the depth, the evaluation points can impact numerical solvers used for seismic wave propagation. In the third constructor above, an array with material layer descriptions is passed along with an array giving the depths of the interface layers between the materials. The latter array should have a length one less than the number of layers. As an example, let us construct a subsurface with a $1 \mathrm{~m}$ thick layer of soil above granite. Referring to the listing 
of SolidIsoLinear from section 5.2.2, SOIL is type 4 and GRANITE is type 10. Hence

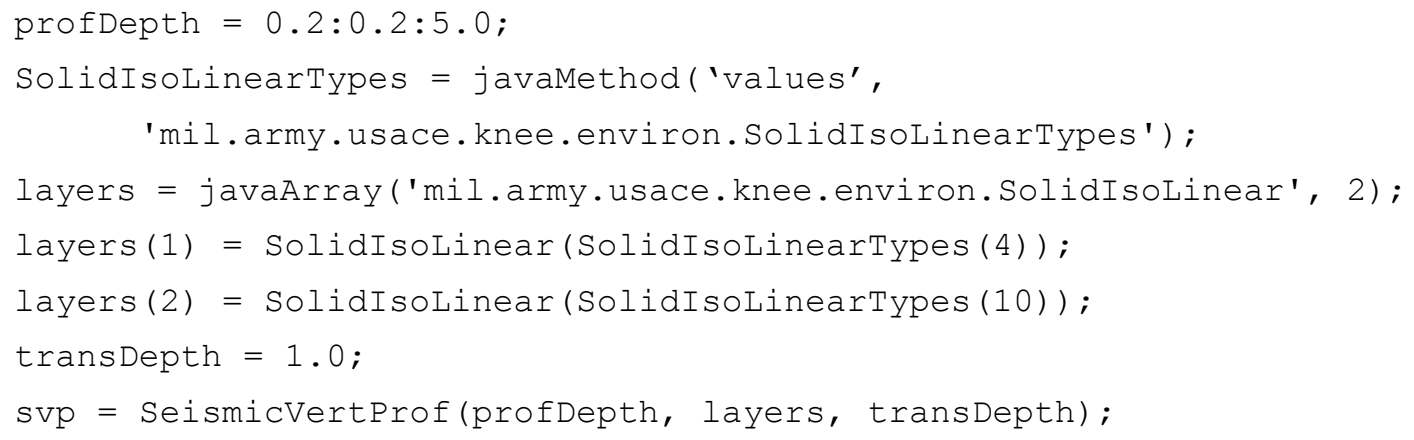

Once the seismic profiles have been constructed, a number of getter methods are available to retrieve their properties, including profiles that are calculated from those specified. The following methods all return double [ ] arrays:

- getProfDepth returns the depths at which the profiles are specified (in meters, where the depth closest to the surface is first and then increases downward).

- getDensityProf returns the density profile.

- getBulkModProf returns the bulk modulus profile.

- getShearModProf returns the shear modulus profile.

- getPWaveProf returns the compressional wave speed profile.

- getSWaveProf returns the shear wave speed profile.

- getPQProf returns the profile of quality factors for compressional waves.

- getSQProf returns the profile of quality factors for shear waves.

For all of the preceding methods with a name ending in Prof, there is also a method where Prof is omitted, which will return the value near the surface. For example, getDensity returns the density at the uppermost profile point. The method getLayer takes an integer argument and returns a SolidisoLinear object corresponding to the profiles at the corresponding profile depth. If the argument is omitted, getLayer returns the uppermost profile point. This can be very useful for obtaining other quantities of interest. For example, getSeismicProf.getLayer.getRayleighSpeed returns the Rayleigh wave speed at the surface. 


\subsection{Environmental representations}

Having discussed the individual components of EASEE's environmental representations, we now put these pieces together into the full product. As mentioned in the introduction to this section, EASEE has two main environmental representations: homogeneous atmosphere and subsurface (EnvironHomo) and vertical profile atmosphere and subsurface (EnvironVertProf). These will now be described.

\subsubsection{Homogeneous environment}

The EnvironHomo class has a great variety of constructor methods, which enable the environmental properties to be specified by various approaches and to varying levels of complexity. The following is one of the simplest constructors, which is pertinent to a flat, homogeneous ground surface:

EnvironHomo(double groundHgt, HumidAir atmos, SolidIsoLinear subsurf)

The arguments are (1) the height of the ground in meters (usually relative to mean sea level); (2) an object representing a humid atmosphere (as discussed in section 5.2.1); and (3) a solid, isotropic, linear object representing the subsurface (as discussed in section 5.2.2). This constructor uses defaults for the land cover (FASSTVegetationTypes.GRASS_SHORT) and soil (FASSTSOIITypes.SAND_CLAYEY), which are assumed to apply throughout the domain.

The following constructor is similar but enables the land cover and soil to be specified explicitly:

EnvironHomo(double groundHgt, Landcover landCov, Soil soil, HumidAir atmos, SolidIsolinear subsurf)

As described in sections 5.2.3 and 5.2.4, respectively, many options are available for specifying particular properties of the soil and land cover.

Consider the following slightly more complicated constructor for EnvironHomo:

EnvironHomo(GeoGridCart2DDouble dem, Landcover landCov,

Soil soil, HumidAir atmos, SolidIsolinear subsurf) 
Compared to the previously considered version of the constructor, the initial argument is a digital elevation model (DEM), in the form of a GeoGridCart2DDouble object, which stores data on a 2-D geographic grid. The DEM is constructed as described in section 4.3.

Lastly, let us consider the following EnvironHomo constructor, which allows specification of land cover and soil that vary across the interface between the ground and air:

EnvironHomo(GeoGridCart2DDouble dem, LandcoverDecoder lcDecoder,

GeoGridCart2DInt lcGrid, SoilDecoder soilDecoder,

GeoGridCart2DInt soilGrid, HumidAir atmos,

Solidisolinear subsurf)

Here, $l_{\mathrm{cDe}}$ coder and soilDecoder are objects specifying the mapping of integer data to land cover and soil types, respectively, as described in section 4.3. After creating lcGrid and soilgrid, we would then construct the desired environment using the command

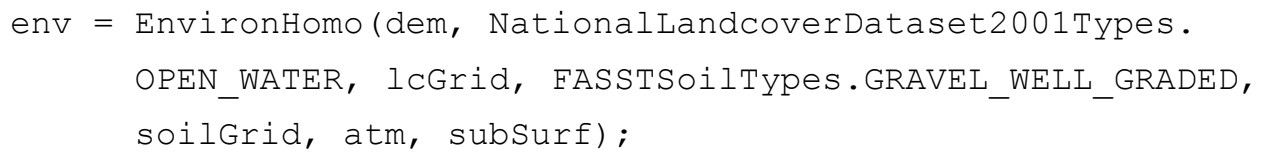

It is important to keep in mind that the specified land cover and soil decoders indicate only which decoding algorithm to use, rather than the actual land cover or soil types (those are specified by lcGrid and soilGrid, respectively). Though NationalLandcoverDataset2001Types.OPEN_WATER and FASSTSOIITypes.GRAVEL_WELL_GRADED were specified above, the decoder can be any valid instance of the Java class for representing the land cover or soil system. In this case, for the land cover, we could have alternatively used NationalLandcoverDataset2001Types.OPEN_WATER, NationalLandcoverDataset2001Types.WETLANDS_WOODY, or any other valid instance of NationalLandcoverDataset2001Types, as opposed to, say, an instance of GeoCoverLCTypes.

Once a homogeneous environment has been constructed, a snow layer and soil moisture may be additionally specified. A single value for the soil moisture fraction throughout the domain may be set using the setSingleSoilmoisture method. The moisture is a value between 0 and 1 , although if a value is specified that exceeds the soil porosity (which can be 
retrieved using FASSTSOilTypes . getPorosity ()), the value will be reduced to the porosity (since the soil moisture fraction cannot exceed the porosity). To set the soil moisture to 0.2, for example, we would enter

env.set.ingleSoilMoisture (0.2);

The setMultisoilMoisture method specifies soil moisture that varies across the domain. Specifically, it takes as input a GeoGridCart2DDouble object, which stores the soil moisture on a geographic grid.

The snow layer includes both the snow type and depth. The setSingleSnowCover method specifies a snow cover that does not vary over the domain. It takes two arguments, snow depth and snow type. For example,

env.setSingleSnowCover(0.1, SnowTypes.SNOW_FRESH);

specifies $0.1 \mathrm{~m}$ of fresh snow throughout the domain. The setmultisnowCover method is used to specify a spatially varying snow cover. It has the form

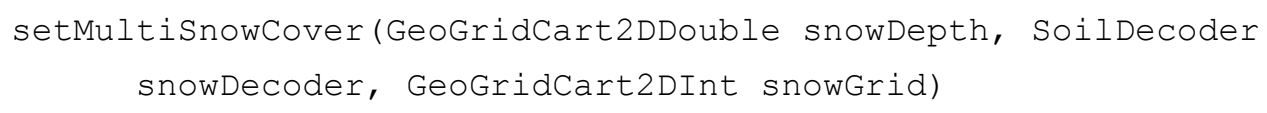

where snowDepth is a geographic grid specifying the snow depth, snowDecoder is an instance of the decoder class for the snow (e.g., SnowTypes.SNOW_FRESH), and snowGrid is a grid of integer codes indicating the snow types.

The EnvironHomo class provides many getter methods that can be used to obtain and display the properties of an object. For example, the DEM is retrieved by entering

demout $=$ env.getDem( $)$;

The method getMeanElev returns the mean elevation for the DEM. The local terrain height can be retrieved at a particular coordinate by specifying a Geocoord as the argument to the getDem method. That is,

disp (env.getDem (coord)) 
displays the terrain elevation at coord, where coord is an instance of Geocoord. The following command displays the elevation at a distance $100 \mathrm{~m}$ north and $200 \mathrm{~m}$ east of the specified coordinate:

disp (env.getDem (GeoCoord (coord, 100.0, 200.0)) )

The getDsm method functions similarly to getDem, except that it pertains to the digital surface map (DSM) rather than the DEM. Here, DSM is the elevation of the bare Earth plus the height of the objects (e.g., buildings and vegetation) on the surface (DEM generally is for the bare Earth.) The getDsm method without argument returns the entire DSM grid, getDsm with a coordinate returns the DSM at that coordinate, and getMeanSurfElev returns the mean for the DSM.

Similar getters are provided for the land cover (getLandcover), soil (getSoil), snow type (getSnow), snow depth (getSnowDepth), and soil moisture (getSoilMoisture). All of these methods can accept a coordinate as an argument, for which they return the value at that coordinate. However, the getLandcover, getsoil, and getsnow methods, when used without an argument, return the most prevalent land cover, soil, and snow type, respectively, since it would make no sense to return a mean value for these quantities. The getLandcoverGrid, getSoilGrid, and getSnowGrid methods return the entire grids. The getSnowDepth and getSoilMoisture methods, without arguments, return the entire grids, whereas getMeansnowDepth and getMeansoilMoisture return the means.

A variety of getters are also available to retrieve the atmospheric and subsurface properties. In this regard, it should be kept in mind that, for the EnvironHomo class, the atmospheric and subsurface properties are constant throughout the domain.

The atmospheric properties are retrieved with the getAtmos method, followed by a getter for the desired quantity. To retrieve the temperature in degrees Celsius and store the result in the variable temp, one would enter

temp $=$ env $\cdot \operatorname{getAtmos}() \cdot \operatorname{getTemp}()$;

Similarly, instead of getTemp, one could use getTempKelvin to get the temperature in Kelvin and getTempFahr to get the temperature in degrees Fahrenheit. Setting 
hum $=$ env $\cdot \operatorname{getAtmos}() \cdot \operatorname{get} \operatorname{SpecHum}() ;$

retrieves the specific humidity, whereas getRelHum, getMolHum, and getMixRat return the relative humidity, molar humidity, and mixing ratio, respectively. The getDensity method returns the air density, getPress returns pressure in Pascals, and getPressmbar returns the pressure in millibar.

The subsurface properties are retrieved with the getsubsurf method, followed by the desired quantity. In particular,

- getDensity returns the density,

- getBulkMod returns the bulk modulus,

- getPWaveSpeed returns the compressional (P-) wave speed,

- getSWavespeed returns the shear (S-) wave speed,

- getRayleighspeed returns the Rayleigh wave speed,

- getPQualFac returns the quality factor for P-waves, and

- getSqualFac returns the quality factor for S-waves.

\subsubsection{Vertical profile environment}

The EnvironVertProf class represents a horizontally stratified environment, that is, an environment in which the atmospheric and subsurface profiles depend on the vertical coordinate only. Internally, EnvironVertProf is the same as EnvironHomo, except that the homogeneous atmosphere and subsurface representations are replaced by representations of the classes AtmosoneDim and SubSurfoneDim. So, most of the description in the previous section regarding EnvironHomo still applies to EnvironVertProf, except regarding setting of the properties of the air and subsurface and retrieval of data using the getAtmos and getsubsurf methods.

The AtmosoneDim class includes the vertical profiles in the atmosphere, specifically, the temperature, specific humidity, pressure, wind speed, and wind direction, which are encapsulated within an AtmosvertProf object (section 5.2.7). AtmosoneDim also includes a representation of the atmospheric surface layer (by an AtmossurfLayer object, section 5.2.6) and cloud layers (by a clouds object, section 5.2.8). Internally, the subsurfoneDim contains the vertical density profile and the seismic vertical profiles of compressional (P-) wave speed, shear (S-) wave speed, and quality factors for P- and S-waves, as represented by a SeismicVertProf object (section 5.2.9). 
Here are three of the most basic and flexible constructor methods for the vertical profile environment:

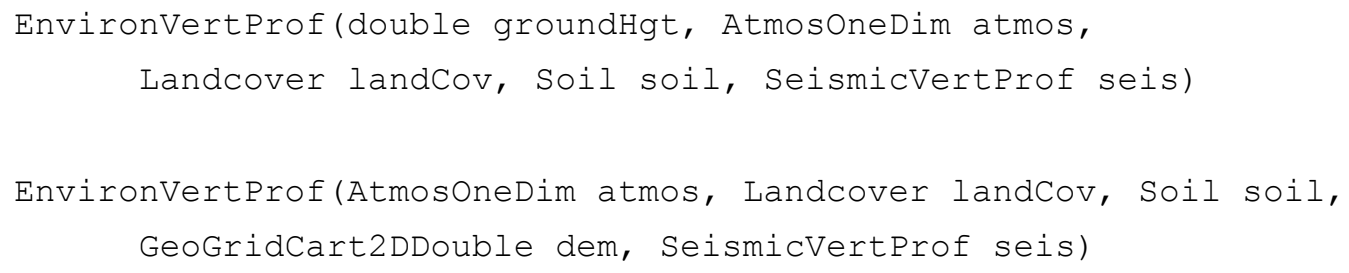

and

EnvironVertProf(AtmosoneDim atmos, GeoGridCart2DDouble dem, LandcoverDecoder lcDecoder, GeoGridCart2DInt landcover, Soildecoder soilDecoder, GeoGridCart2DInt soil, SeismicVertProf seis)

The first of these constructors assumes flat ground and no terrain. The second and third allow the DEM to be explicitly specified. Furthermore, the first and second constructors apply to an environmental model in which the land cover and soil properties are the same throughout the domain; the third allows them to vary horizontally. Construction of the DEM, land cover, and soil objects appearing in these constructors follows from the discussion in the previous subsections. The new aspect of these constructors is the AtmosoneDim object. (Because the SubsurfoneDim is basically just a wrapper around the seismicVertProf class, the constructors take the seismicVertProf object directly.)

AtmosOneDim has a rich variety of constructors, which enables the atmospheric profiles to be specified directly by certain benchmark cases or by MOST. These constructors are often quite similar to AtmosVertProf; however, using the AtmosoneDim constructor is preferable in such cases to ensure that the AtmosSurfLayer and clouds objects are constructed in a manner that is compatible with the vertical profiles.

The following two constructors specify the vertical profiles and clouds explicitly while inferring information about the surface layer:

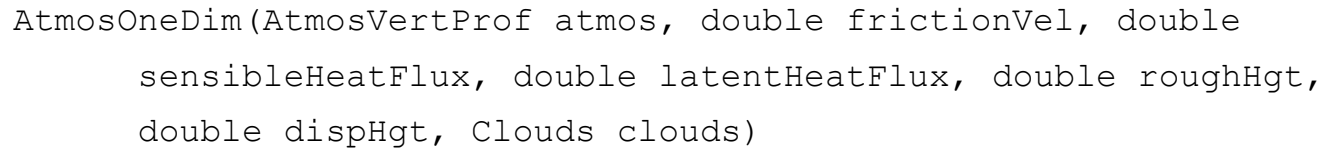


or

AtmosoneDim(AtmosVertProf atmos, double roughHgt, double dispHgt, Clouds clouds)

For both forms, one would ordinarily construct the profiles using one of the methods in section 5.2.7 and the clouds using one of the methods in section 5.2.8. The first form specifies the vertical profiles directly as an AtmosVertProf object and builds the surface layer from the specified friction velocity $(\mathrm{m} / \mathrm{s})$, sensible heat flux $\left(\mathrm{W} / \mathrm{m}^{2}\right)$, latent heat flux $\left(\mathrm{W} / \mathrm{m}^{2}\right)$, roughness height $(\mathrm{m})$, and displacement height $(\mathrm{m})$. The lowermost height in the profiles is used to determine the surface temperature and humidity. The second form is similar but infers the friction velocity and heat fluxes by applying MOST to the two lowermost heights of the profiles.

The following constructor yields an atmosphere based solely on profiles constructed from MOST:

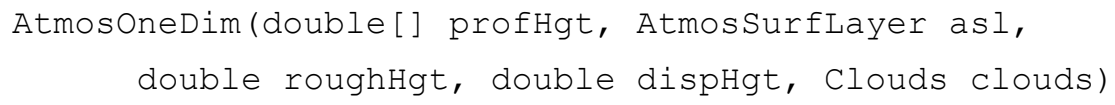

Here, Atmossurftayer is the class for representing atmospheric surface layers, as described in section 5.2.6. The arguments roughHgt and dispHgt are the surface roughness and displacement heights, respectively. They may be retrieved from the land cover by using the getRoughHgt and getDispHgt methods.

AtmosoneDim also has a constructor for benchmark profiles, which is of the same form as that for AtmosVertProf (section 5.2.7), namely

AtmosoneDim(double[] profHgt, AtmosVertProf.BenchmarkProfileTypes
proftype)

The previous section discussed a number of getter methods with regard to the EnvironHomo class that enable examination of the properties of the environment. Those methods generally apply to EnvironVertProf as well, with the main exceptions being getAtmos and getsubsurf (methods return objects representing the 1-D atmosphere and subsurface, respec- 
tively, as opposed to homogeneous representations). Some methods specific to EnvironvertProf are also defined in this class, namely getAtmossi, getAtmosProf, getclouds, and getseismicProf, which return the atmospheric surface layer, atmospheric profiles, clouds, and seismic profiles, respectively. One can then append additional getter methods to obtain the quantity of interest. For example, if env is an object of the EnvironVertProf class, env.getAtmosSL.getFrictionVel would return the friction velocity.

\subsection{Loading WRF Data}

As described in section 5.3.2, EASEE supports a great many methods to construct vertical profile environments. However, these all assume that the user has already obtained information on the atmosphere from other sources. We previously circumvented the question of what that source might be. In this subsection, we focus on a method that enables EASEE to ingest a particular source of atmospheric data, namely output from the Weather Research and Forecasting (WRF) weather model.

The WRF model (with the Advanced Research WRF [ARW] solver) is a regional weather model that solves fully compressible, Eulerian, nonhydrostatic equations (Skamarock et al. 2008) and can produce atmospheric forecasts at a variety of horizontal, vertical, and temporal resolutions. It also includes many parameterization options for planetary boundary-layer physics, surface physics, and other atmospheric domains. Because WRF is a community model, it is free and publicly available at the WRF Model Users' Page (NCAR 2018) and is used widely by the atmospheric community, with over 39,000 users in over 160 countries (NCAR 2017). Overall, WRF's versatility and large user base make it desirable as a source of atmospheric data for EASEE.

EASEE supports the usage of either (1) a single WRF output file or (2) an ensemble of WRF output files (i.e., a set of model output files from nearly identical simulations differing only in the initial or boundary conditions). The following two subsections describe the procedures for working with single or ensemble forecast files, respectively.

\subsubsection{Single WRF Output File}

To use data from the WRF output file's native format-NetCDF (Network Common Data Format)-EASEE uses methods from the NetCDF-Java API 
v4.6. This API requires the creation of a Java-NetCDF object (NetcdfFile) from the WRF output file that can be readily used within EASEE. To do this, first open the WRF output file in MATLAB:

ncfile = WrfLoader.openNetcdf(fileLocation);

where fileLocation is the path to the desired WRF output file and ncfile is the Netcdffile object derived from the WRF output file that can be used within EASEE.

Generally, users will provide their own WRF files for use within EASEE. However, there are also some example WRF files that can be accessed through the online GitLab repository that contains EASEE; KNEE can be cloned from this repository, and the WRF test files can then be obtained. Please contact the authors for more information about accessing the GitLab repository.

If you would like to use the single WRF test file and you have cloned the KNEE portion of the EASEE GitLab repository, then the test file can be found at the following file path:

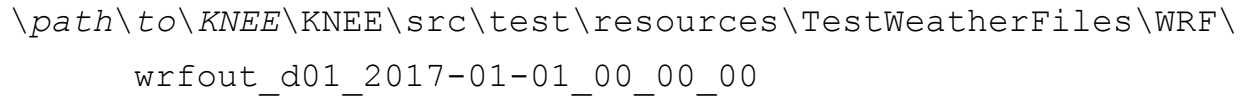

where $\backslash p a t h \backslash t o \backslash K N E E \backslash$ is the path to the EASEE/KNEE directory on your local machine. The test file specified by the above path has 17 time steps, with output at 3-hour intervals over 2 model days (from 2017.01.01 at oooo UTC [Coordinated Universal Time] to 2017.01.03 at oooo UTC). The simulation domain has a $5 \mathrm{~km}$ horizontal resolution and 29 vertical model layers (model top is at $100 \mathrm{hPa}[10 \mathrm{kPa}]$ ). The domain is centered at $40 .{ }^{\circ} \mathrm{N}, 75{ }^{\circ} \mathrm{W}$ and spans 150 grid cells west-east and 120 grid cells southnorth.

If you would like to use your own WRF output file instead, or if you receive the above test file through means other than cloning the GitLab repository, simply substitute the appropriate path to the file for fileLocation.

For the case of a single file, the wrfLoader class is used to construct an object that extracts weather data from a WRF output file. WrfLoader extends the AtmosoneDim class and thus comes preequipped with default versions of 
AtmosVertProf, Clouds, and AtmosSurflayer objects. Specifying this constructor updates these default objects by using data from the WRF output file, allowing a more accurate AtmosoneDim object to be created.

There is one main constructor available for WrfLoader:

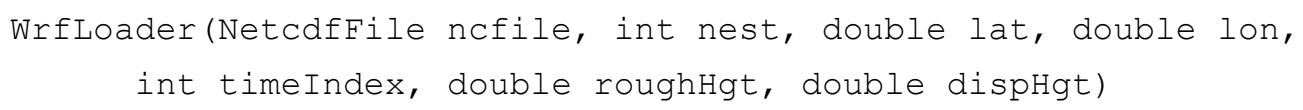

This constructor requires a WRF output file and the approximate location in latitude $\left(-90^{\circ}\right.$ to $\left.90^{\circ}\right)$ and longitude $\left(-180^{\circ}\right.$ to $\left.180^{\circ}\right)$ at which you would like to extract a vertical atmospheric profile. The latitude/longitude values are specified in decimal degrees. Alternatively, a variation of the constructor allows you to instead specify the latitude and longitude indices (e.g., 1, $2,3, \ldots$ ) corresponding to the grid cell numbers in the south-north and west-east directions, respectively. WRF simulations can be conducted with multiple nested horizontal domains (e.g., with a parent domain at $9 \mathrm{~km}$ horizontal resolution and a smaller, nested domain at a finer $3 \mathrm{~km}$ horizontal resolution). The variable nest refers to the horizontal model domain in which you would like to sample a vertical profile (i.e., the parent domain would be 1 , the next nested domain would be 2, etc.). (Note that nest $=\mathbf{1}$ for a WRF simulation with a single domain, such as the aforementioned WRF test file). Finally, roughHgt and dispHgt represent the roughness height ( $\mathrm{m}$ ) and displacement height (m), respectively.

The following is an example of loading data from the single WRF test file into EASEE and plotting vertical profiles of air temperature and wind.

First, import KNEE's environ and geo packages:

import mil.army.usace.knee.environ. *;

import mil.army.usace.knee.geo. *;

Next, open the WRF test file using its path on your local machine:

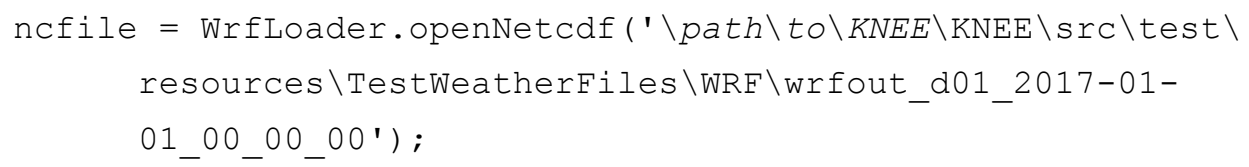


where $\backslash p a t h \backslash t o \backslash K N E E \backslash$ is the path to the EASEE/KNEE directory on your local machine.

At this point, you can test whether your chosen latitude and longitude values (e.g., 40, -75) fit within the estimated boundaries of the domain. This can be accomplished by running WrfLoader.getWrfLatLonIndices (ncfile, 40 , -75 ). You must adjust your latitude/longitude values until the last line of output from this command reads, for example, closest lat/lon indices: $(59,74)$, where 59 and 74 are the vertical and horizontal positions of the closest grid cell, respectively.

Then, use the WrfLoader constructor to initiate an object with WRF data:

wrffile = WrfLoader(ncfile, 1, 40, -75, 1, 0.1, 0.2);

where 1 refers to the chosen domain within the WRF simulation (only one domain in this file, so nest $=1), 40\left(40^{\circ} \mathrm{N}\right)$ is the chosen latitude, -75 $\left(75^{\circ} \mathrm{W}\right)$ is the chosen longitude, 1 is the second time step (o-based Java indices), 0.1 is the roughness height in meters (typical for grassland), and 0.2 is the displacement height in meters (estimated as twice the roughness height).

The following output should be generated after running the wrfLoader constructor:

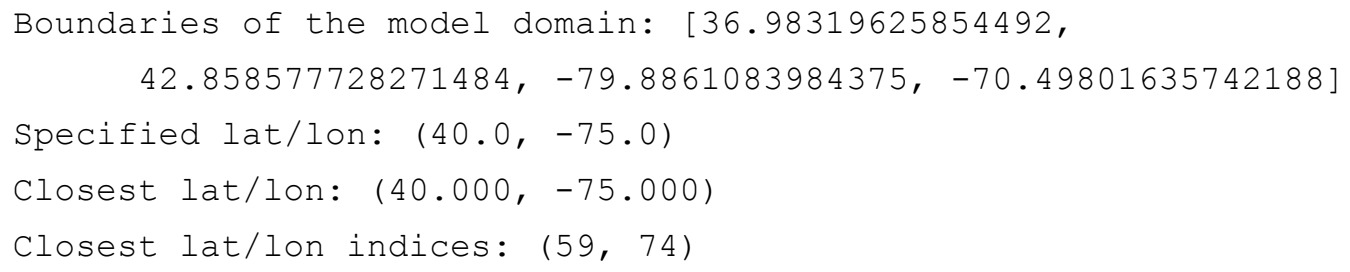

Additionally, typing wrfFile at the MATLAB command prompt will print summaries of the objects for the atmospheric surface layer, atmospheric vertical profiles, and clouds:

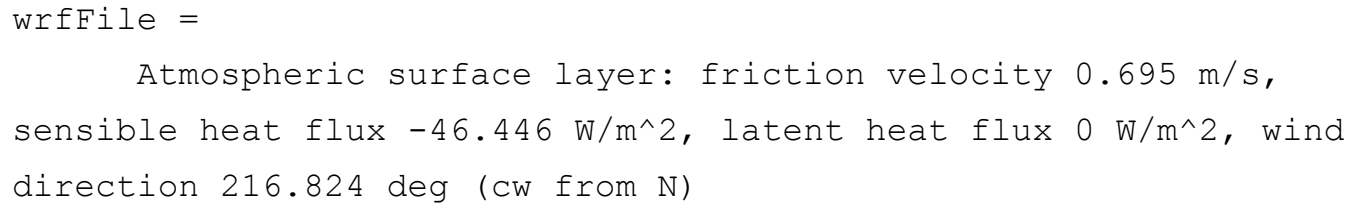


Atmospheric vertical profiles: 29 profile points, inversion height $732.0818481445312 \mathrm{~m}$

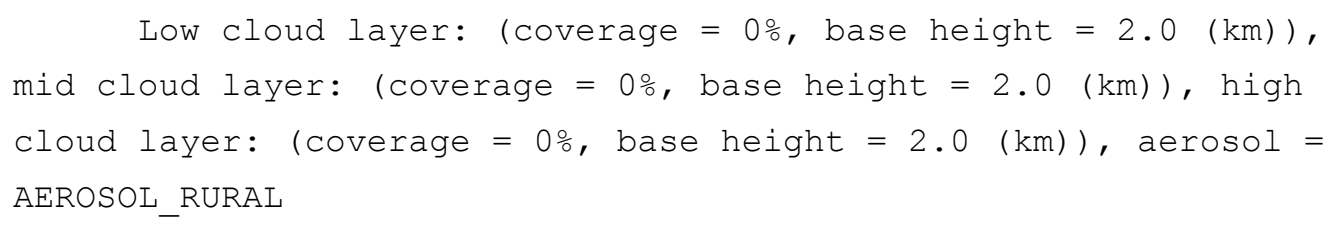

Now that the WRF data have been successfully imported, use methods from EASEE to extract the vertical profiles of temperature and wind, and then use MATLAB commands to plot them:

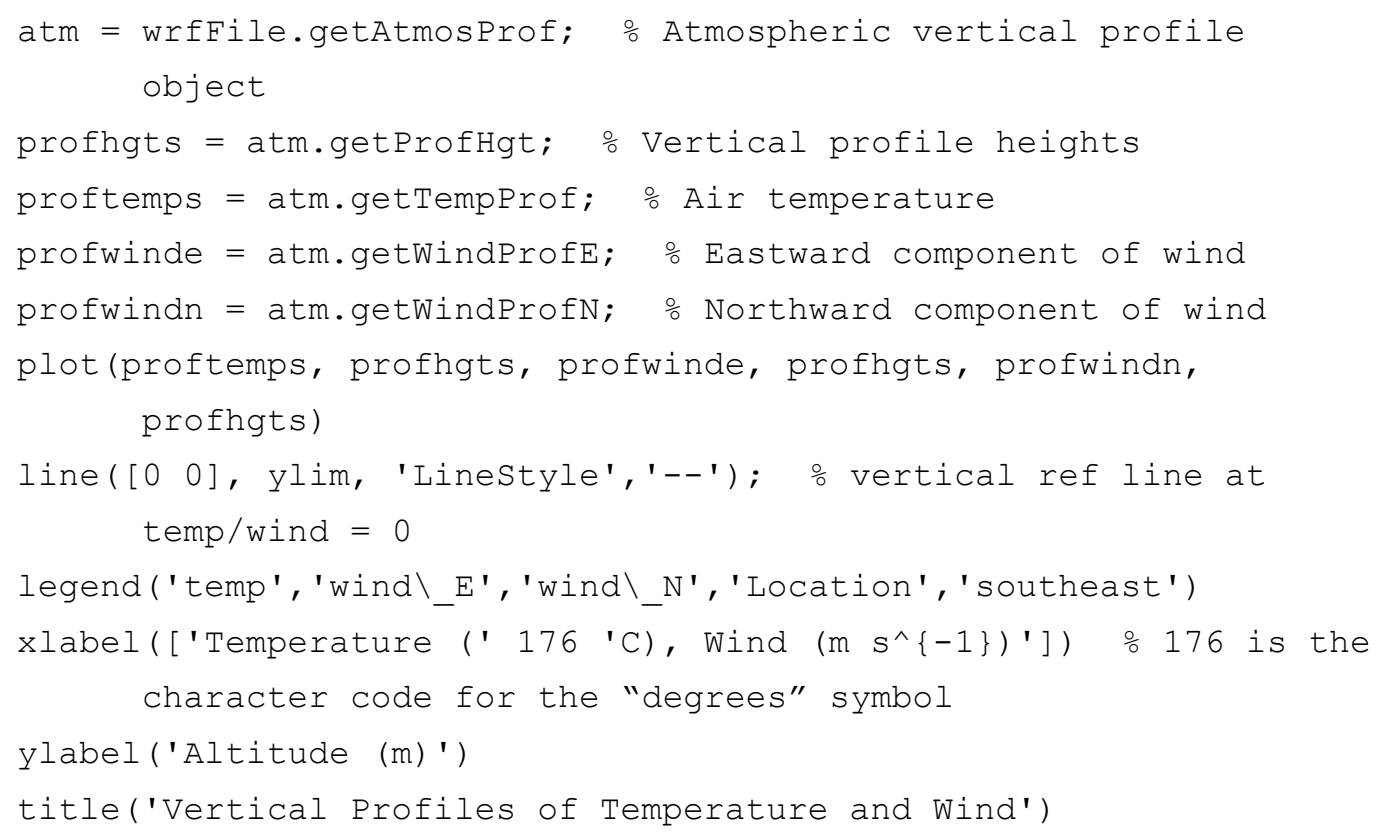

The above block of code should produce Figure 5 .

Alternatively, you can plot wind speed and/or wind direction. Simply substitute the following lines into the larger chunk of code above and adjust the variable names and axis labels:

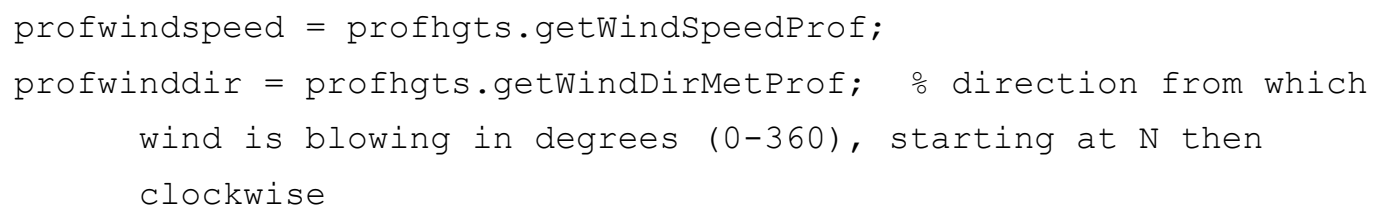


Figure 5. Vertical profiles of air temperature (b/ue), the eastward wind component (red), and the northward wind component (orange) derived from a WRF test file in the EASEE repository.

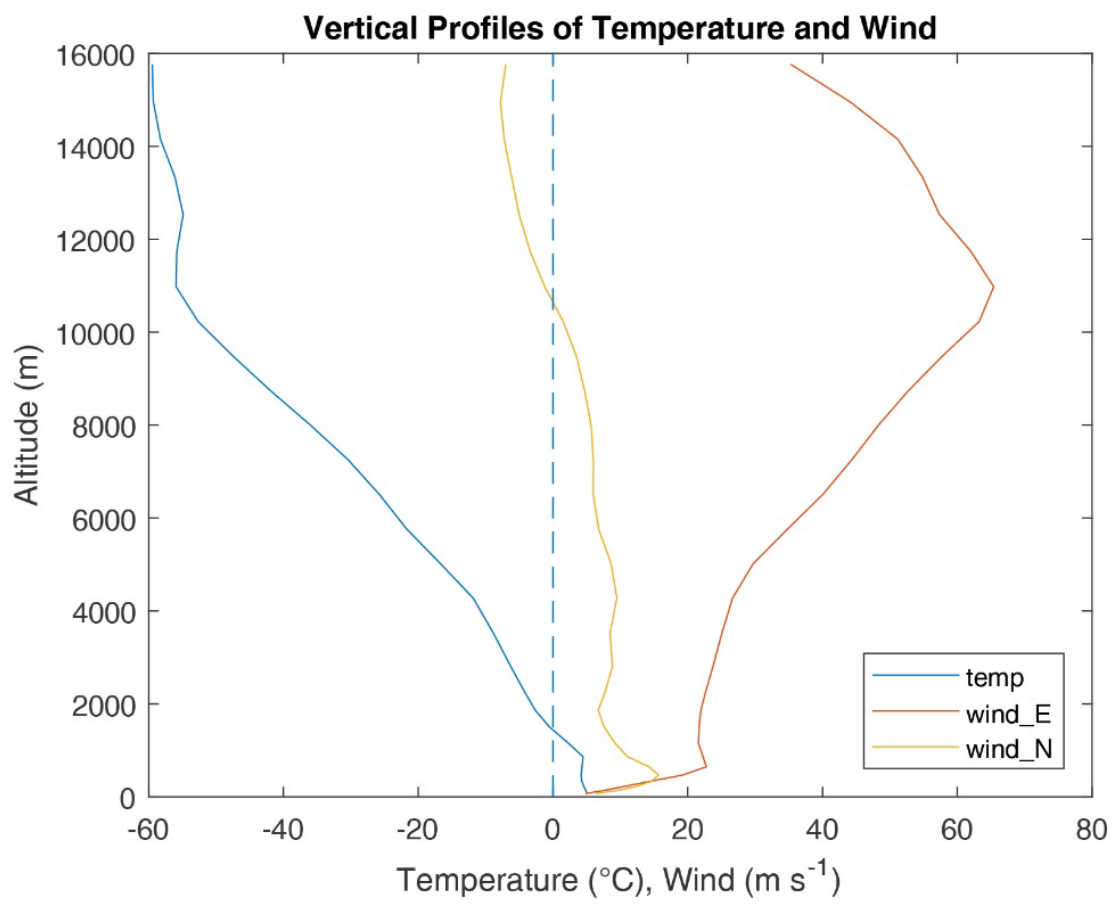

\subsubsection{Multiple WRF output files}

If you would like to instead use multiple WRF output files (i.e., a forecast ensemble), then first identify the path of each WRF output file, and open the files in MATLAB using the following commands:

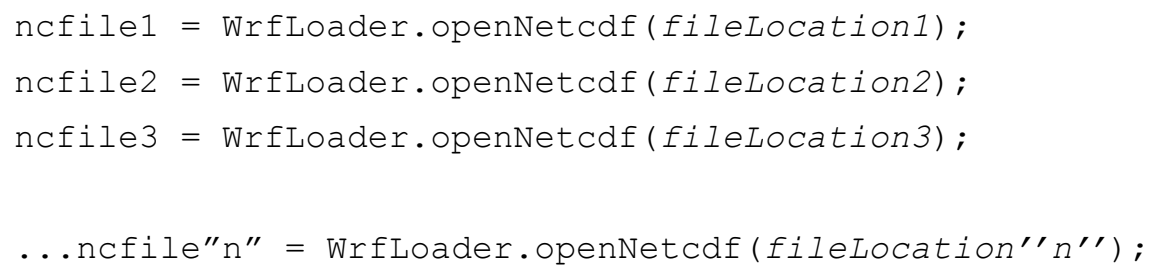

where $f$ ileLocation $1,2, \ldots n$ are the paths to the desired WRF output files and ncfile1,2, ..n are the Netcdffile objects derived from the WRF output files that can be used within EASEE.

As with the single WRF output file case, users will generally provide their own WRF files for use within EASEE. However, there are also some example WRF files within the EASEE repository that can be used for testing purposes. 
If you would like to use the multiple WRF test files and you have cloned the KNEE portion of the EASEE GitLab repository, then the test files can be found at the following file paths:

$\backslash p a t h \backslash$ to $\backslash K N E E \backslash K N E E \backslash S r C \backslash$ test $\backslash$ resources $\backslash$ TestWeatherFiles $\backslash W R F \backslash$ wrfout_d01_2017-01-01_00_00_00_file\#

where $\backslash p a t h \backslash t o \backslash K N E E \backslash$ is the path to the EASEE/KNEE directory on your local machine and where \# refers to an integer from 1 to 3 . The three test WRF files located at the above file paths are "pseudoensemble" members (i.e., they are different temporal subsets of the original file, wrfout_do1_2017-01-01_00_00_00).

The test files specified by the above path each have four time steps, with each file containing output at 3-hour intervals that sequentially cover 1.5 model days (i.e., file1 is 2017.01.01 at 0000, 0300, 0600, and 0900 UTC; file2 is 2017.01.01 at 1200, 1500, 1800, and 2100 UTC; etc.). All of the other domain metadata are the same as those for the original wrfout_do1_2017-01-01_0o_00_oo file from the "Single WRF Output File" section.

If you would like to use your own WRF output files instead, or if you receive the above test files through means other than cloning the GitLab repository, simply substitute the appropriate paths to the files for fileLocation $1,2, \ldots$.

After the WRF output files have been opened, you can use the Wrfensemble constructor, which constructs an environmental ensemble from a list of WRF output files:

WrfEnsemble (List<Netcdffile> ncfile, int nest, int latIndex, int
lonIndex, int[] timeIndex, EnvironVertProf env)

This constructor is similar to the WrfLoader constructor in that WRF output data are specified along with the location at which vertical atmospheric profiles should be extracted. However, the main differences are that ncfile in this constructor consists of multiple NetcdfFile objects, the time Index field is an array of time steps rather than a single time step, and a default EnvironVertProf object is specified instead of other component parts such as roughHgt. Furthermore, this constructor formulation re- 
quires latitude and longitude indices corresponding to the grid cell numbers in the south-north and west-east directions, respectively, rather than exact values of latitude and longitude. These are obtained using a method called getWrfLatLonIndices, which is described later in this section.

Similar to the WrfLoader constructor, Wrfensemble extends the EnvironModelEnsemble class and thus contains a default ensemble (EnvironEnsemble) of EnvironvertProf objects. Specifying this constructor updates the component parts of these default EnvironVertProf objects using data from the WRF output files, allowing more accurate EnvironVertProf objects to be created.

The following is an example of loading data from multiple WRF test files into EASEE and plotting vertical profiles of air temperature from each "ensemble" member (noting that the three files are actually "pseudoensembles" derived from the same original simulation).

First, import KNEE's environ and geo packages and Java's ArrayList package using the following commands:

import mil.army.usace.knee.environ. *;

import mil.army.usace.knee.geo. *;

import java.util.Arraylist;

Next, open the three WRF test files using their paths on your local machine:

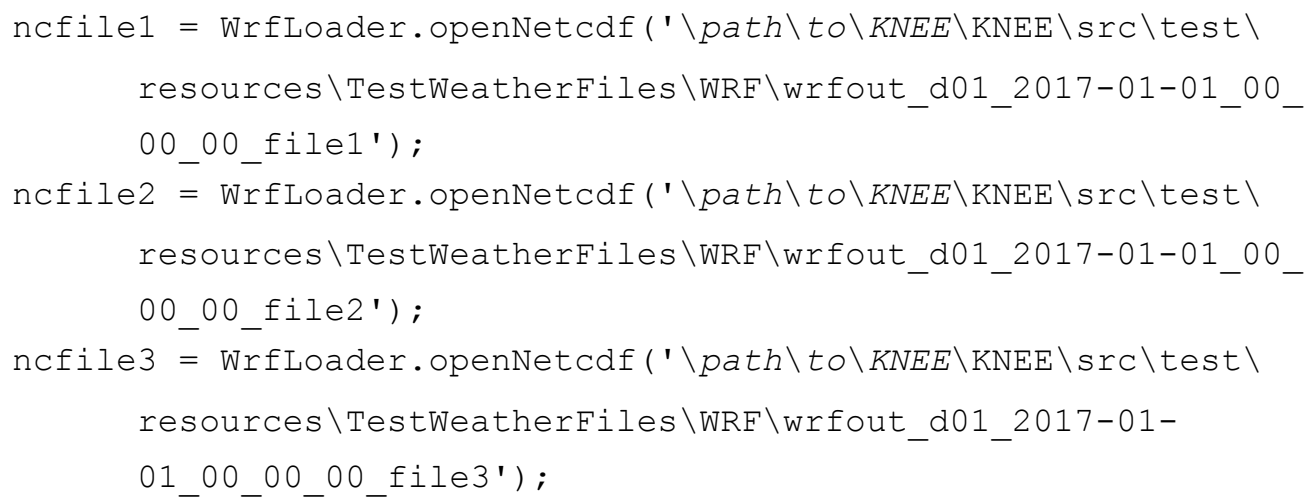

where $\backslash p a t h \backslash t o \backslash K N E E \backslash$ is the path to the EASEE/KNEE directory on your local machine. 
Then, create a Java ArrayList to hold the multiple WRF files. The WRF files are added to the ArrayList one at a time:

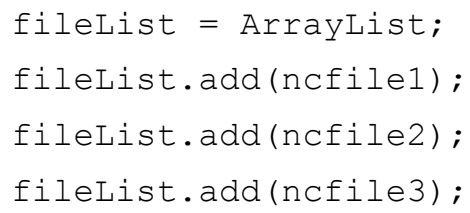

The WrfEnsemble constructor requires several parameters that must be created before the constructor is used. First, instantiate a default EnvironvertProf object:

env = EnvironVertProf ();

Next, you can use an EASEE method called getWrfLatLonIndices to convert your chosen latitude and longitude values to integer indices on a grid:

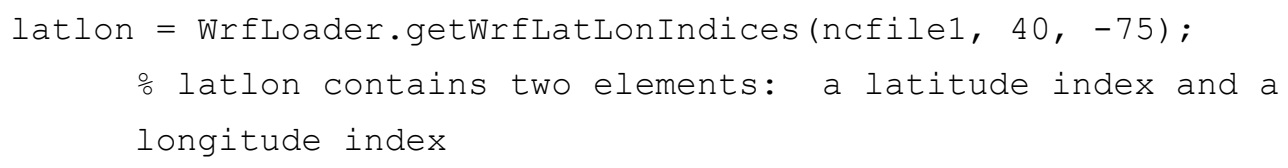

Note that the output from the above command may indicate that your chosen latitude/longitude values are not within the estimated bounds of the simulation domain. You must adjust your latitude/longitude values until the last line of output from this command reads, for example, closest lat/lon indices: $(59,74)$.

Finally, determine the number of time steps available in the WRF files so that you do not accidentally use time indices that are out of range:

timesize = WrfLoader.getTimeSize(ncfilel);

If you type timesize into the MATLAB command prompt, you will see that there are four time steps in each WRF file. Thus, your Java-based time indices must be within the range $[0,3]$, inclusive.

Now you can use the Wrfensemble constructor with the above parameters:

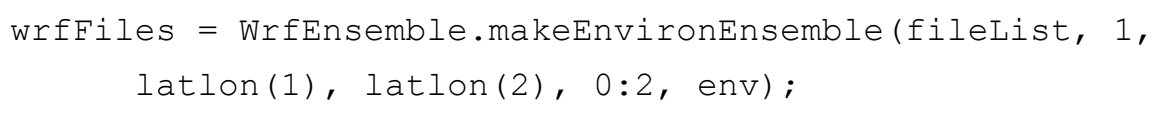


where 1 refers to the chosen domain within the WRF simulations (only one domain in these files, so nest =1); latlon(1) and latlon(2) are the grid indices of latitude and longitude, respectively; and o:2 are indices of the time steps (first through third) to be sampled from the original files.

From this WrfEnsemble object, you can extract separate ensemble members:

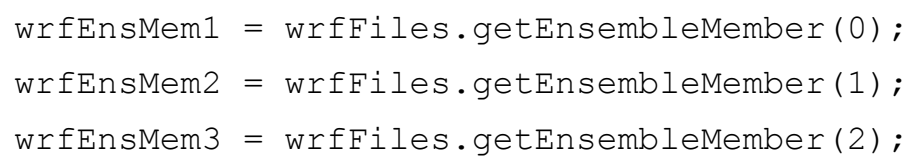

The three wrfEnsMem variables above represent the three ensemble members within the Wrfensemble set of simulations.

After the individual files have been extracted, it is easier to analyze the WRF data. Now you can plot temperature profiles for each ensemble member:

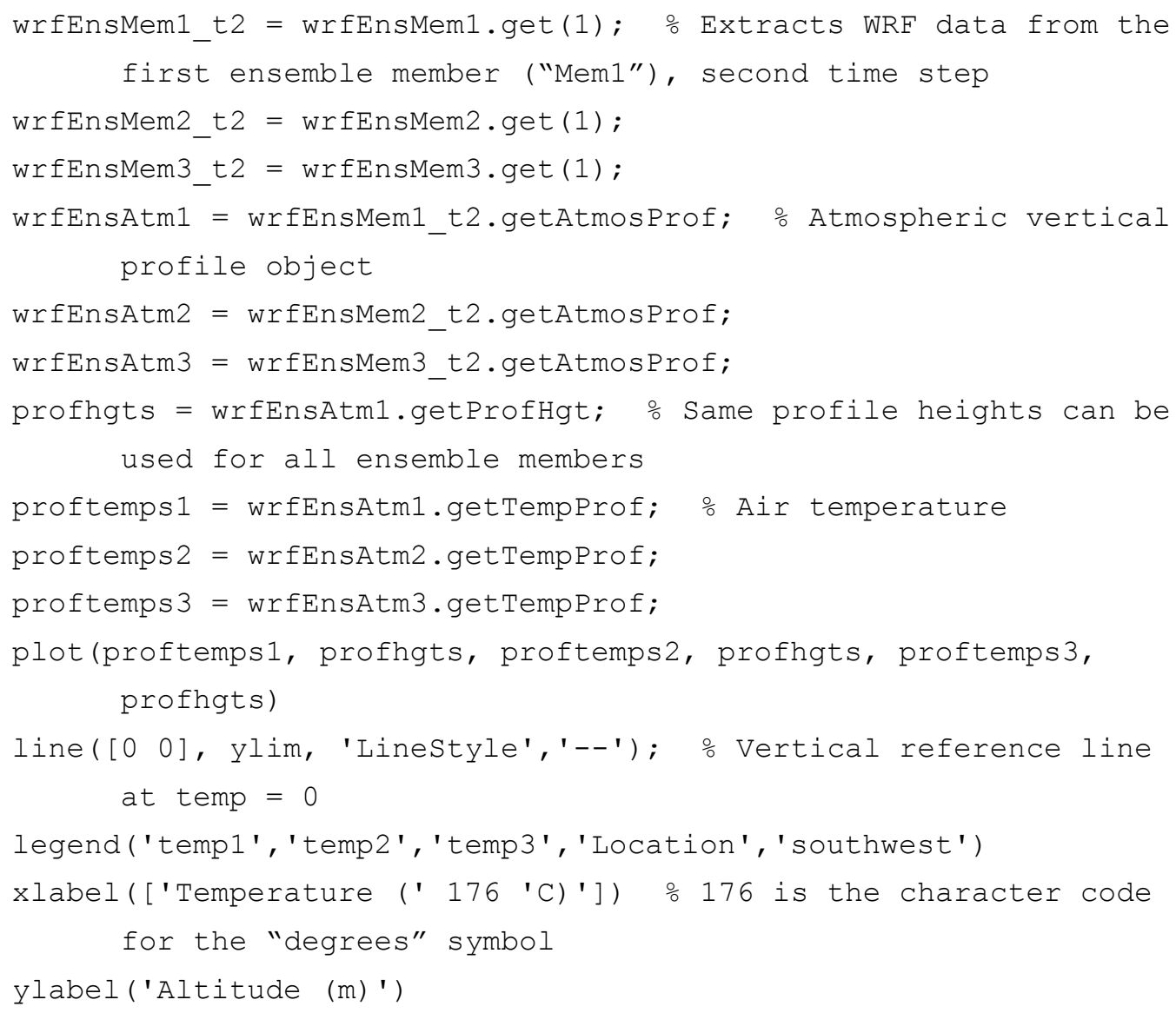


title('Vertical Profiles of Temperature for Three "Ensemble"
Members')

The preceding block of code should produce Figure 6 .

Figure 6. Vertical profiles of air temperature derived from three pseudoensemble test WRF files in the EASEE repository.

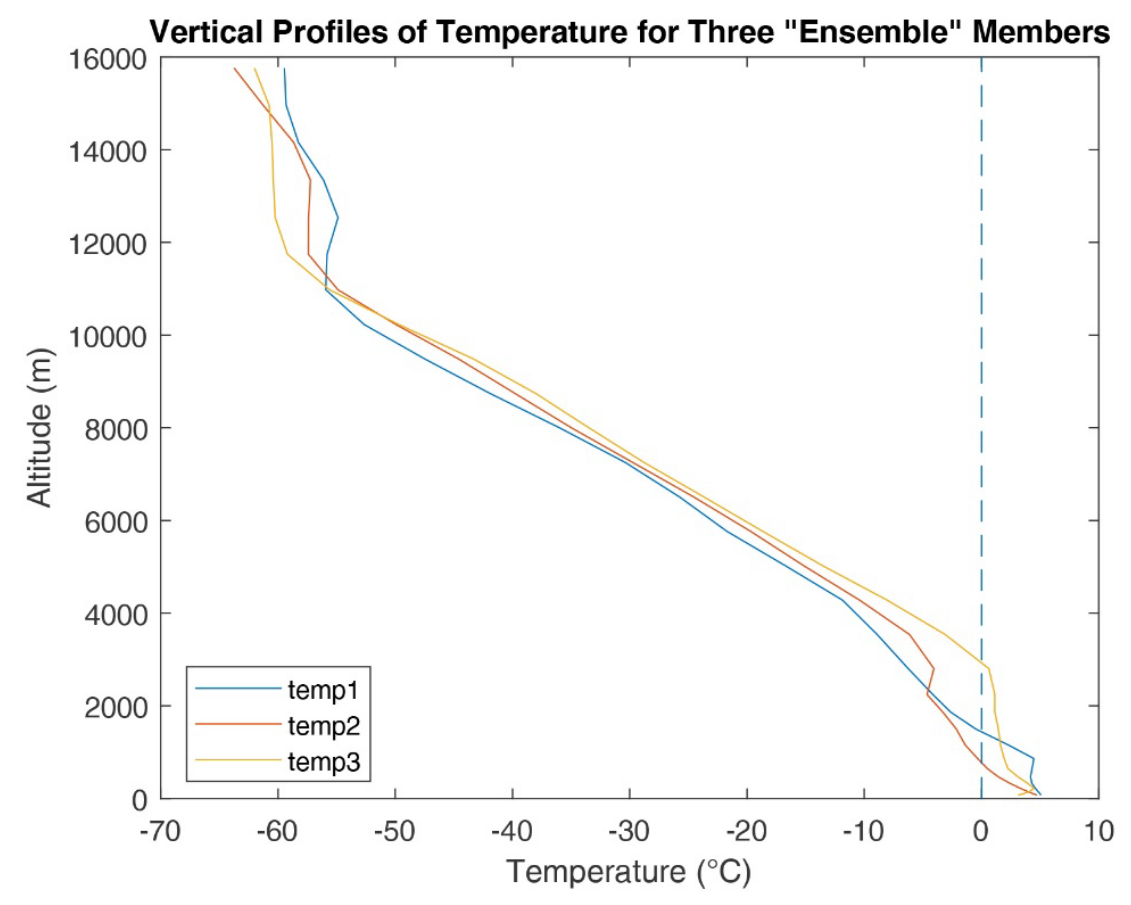




\section{Acoustic Propagation Calculations}

KNEE's acoustic package provides a number of data representations and basic calculations related to acoustics. These include, for example, calculation of air absorption, porous media models for ground impedance, and calculation of the sound field above an impedance ground. Assuming the StartEASEE.m script has been run successfully (section 3.1), the KNEE acoustic package can be imported into MATLAB with the following command:

import mil.army.usace.knee.acoustic. *;

More advanced and militarily sensitive capabilities, such as the National Aeronautics and Space Administration (NASA) Green's Function Parabolic Equation (GFPE) method, are found in the EASEELib acoustic package, which is imported with the following command:

import mil.army.usace.easee.acoustic. *;

We begin this section with a discussion of several useful preliminaries: namely, how to construct standard octave and one-third octave bands, how to construct acoustic media for air and porous media, and how to set up calculation grids to store the model output. We will then consider several particular propagation models. Lastly, we will discuss an alternative approach to performing acoustical calculations, which involves constructing the environmental properties using EASEE and then performing the actual propagation calculations in MATLAB.

\subsection{Standard acoustic frequencies}

KNEE's spectra package contains Java classes for representing and manipulating power spectra. Although we will not describe the functionality of this package in detail here, we do describe one particularly useful feature, namely the availability of standard frequencies for octave and onethird octave bands. To access these frequencies, first import the BandedPowerLawSpec class:

import mil.army.usace. knee.spectra.BandedPowerLawSpec;

Then, to retrieve standard octave band center frequencies, for example, enter 
freq $=$ BandedPowerLawSpec.STANDARD_OCTAVE_CENTER_FREQS;

This will create a MATLAB array containing all of the standard frequencies. To retrieve standard one-third octave-band center frequencies, enter

freq $=$ BandedPowerLawSpec.STANDARD_ONE_THIRD_OCTAVE_CENTER_FREQS;

Similarly, the STANDARD_OCTAVE_LOWER_FREQS and STANDARD_OCTAVE_UPPER_ FREQS provide the lower and upper frequency bounds for the standard octave bands, whereas STANDARD_ONE_THIRD_OCTAVE_LOWER_FREQS and STANDARD_ ONE_THIRD_OCTAVE_UPPER_FREQS provide the analogous results for one-third octave bands.

\subsection{AcousticMedium class}

The AcousticMedium class plays an important role in EASEE's implementation of acoustics by enabling the construction of the acoustical properties (impedance, complex wave number, attenuation, phase speed, etc.) of media. In particular, EASEE's models for the acoustical properties of air and for porous media are extensions of this class.

To use the acoustic medium class, you will first need to import the Apache Commons complex-number class by typing

import org.apache.commons.math3.complex.Complex;

This class is needed because Java, unlike MATLAB, does not directly support complex numbers. The AcousticMedium class defines methods to retrieve

- the characteristic impedance (get Imped and getNormImped, for the unnormalized impedance and the impedance normalized by the reference value of $\rho_{\mathrm{o}} c_{0}$, where $\rho_{\mathrm{o}}$ is the reference density and $c_{\mathrm{o}}$ is the reference sound speed);

- the characteristic admittance (getAdmit and getNormAdmit);

- the characteristic resistance (getResist and getNormResist);

- the characteristic reactance (getReact and getNormReact);

- the complex wavenumber (getWaveNumber and getNormWavenumber, the latter being normalized by $2 \pi f / c_{0}$, where $f$ is the acoustic frequency);

- the phase speed (getPhaseSpeed); 
- the attenuation coefficient in $\mathrm{Np} / \mathrm{m}$ (getAtten);

- the complex bulk modulus (getCompBulkMod); and

- the complex density (getCompDensity).

The methods for the impedance, admittance, complex wavenumber, complex bulk modulus, and complex density return complex values of the Apache complex number type. The methods for resistance, reactance, phase speed, and attenuation return real values, which can be more convenient to manipulate in MATLAB.

All of the previously described methods can accept either a single value for the frequency or an array of values. The output would correspondingly be a single value or an array of values providing results for each specified frequency.

The AirMedium class is an extension of AcousticMedium, which provides the acoustical properties of air at a particular temperature, humidity, and pressure. In this regard, AirMedium is complementary to the HumidAir class in the environ package, which was discussed in section 5.2.1. In fact, one can construct an AirMedium object either by specifying the air properties directly or by passing a HumidAir object:

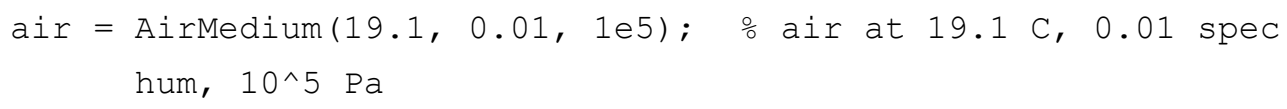

Or

air = AirMedium(HumidAir(19.1, 0.01, 1e5));

The two preceding constructors produce equivalent results. The pressure can be omitted from either one, in which case sea-level pressure is assumed. With the second form, any of the HumidAir constructors described earlier in this report may be used.

Once the AirMedium has been constructed, we can call the methods previously described for the AcousticMedium class. For example, the following code creates a plot of attenuation between the frequencies of $20 \mathrm{~Hz}$ and $20 \mathrm{kHz}$ for air at $18.1^{\circ} \mathrm{C}, 40 \%$ relative humidity, and sea-level pressure: 


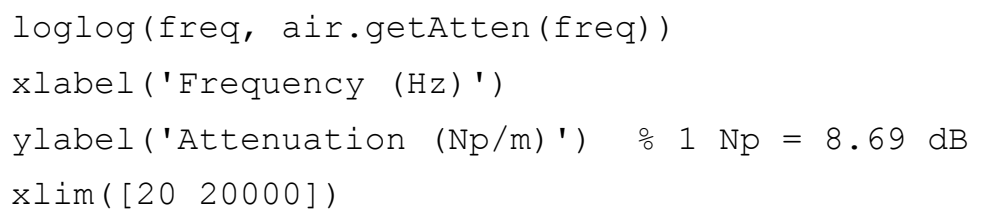

Figure 7 shows the result.

Figure 7. Attenuation coefficient for air at $20^{\circ} \mathrm{C}, 40 \%$ relative humidity, and sea-level pressure.

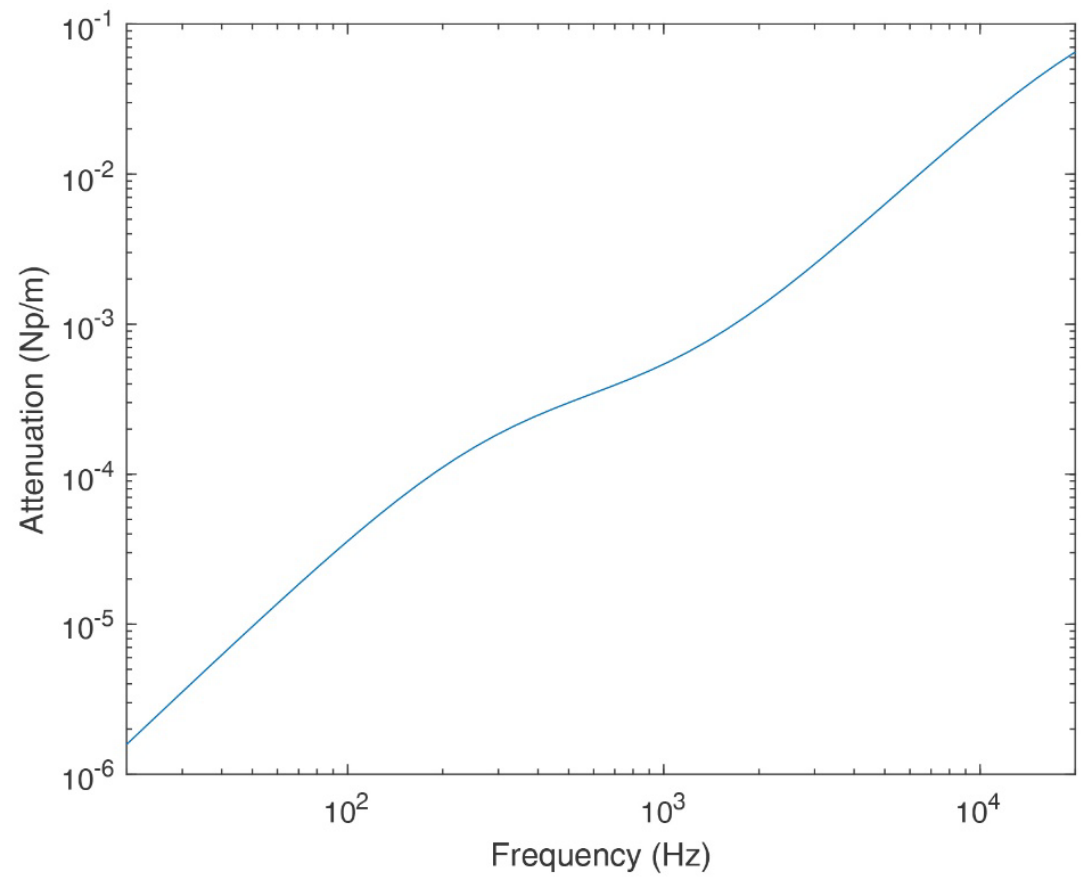

The AcousticMedium class also serves as the basis for EASEE's representations of the acoustical properties of porous media. The models currently supported are Attenborough's four-parameter model (AttenboroughFourParamMedium), the Delany-Bazley model (DelanyBazleyMedium), the relaxation model (RelaxMedium), and the Zwikker-Kosten model (ZwikkerKostenMedium).

As an example, let us consider the Zwikker-Kosten model, which represents the porous medium using three parameters: static flow resistivity, porosity (void fraction), and the so-called structure constant, which is related to pore tortuosity. Two primary constructors are provided:

and 
ZwikkerkostenMedium(double ks, double por, double flowres)

The first of these constructors accepts the porosity (a fraction between 0 and 1) and static flow resistivity and then estimates the structure constant based on the porosity. The second allows all three parameters to be set directly.

Information on the other porous models and their constructors can be found in the Javadoc or by directly examining the Java code.

The BenchmarkMedium class, which extends AcousticMedium, sets the ground properties as appropriate to certain acoustical benchmark calculations found in Attenborough et al. (1995) and Di and Gilbert (1993).

\subsection{Calculation grids}

EASEE calculations are performed on a grid (array) of specified source and receiver positions. The structure of the grid depends on the underlying symmetry of the underlying propagation calculation. Two main types of structured grids are of interest for running the acoustic propagation models: vertical and polar. The vertical grid is used for models for which the output depends on source height, receiver height, and horizontal range between the source and receiver. The polar grid introduces a dependence on the azimuth (e.g., the direction relative to the wind). For both the vertical and polar grids, the horizontal source position is assumed to be zero (meters for the vertical grid, degrees for the polar grid).

Let us consider an example for setting up a vertical grid. In this example, the grid has a single source height of $5.0 \mathrm{~m}$ and a single receiver height of $1.0 \mathrm{~m}$, and the range varies from $\mathrm{o} \mathrm{m}$ to $1000 \mathrm{~m}$ in increments of $10 \mathrm{~m}$ :

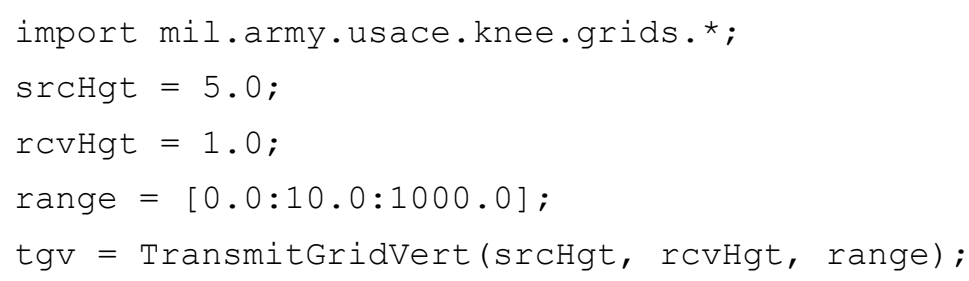

The polar grid is constructed with one additional argument, representing the azimuth in radians. By convention, the azimuth is bounded between $-\pi$ and $\pi$. The following code sets up a polar grid with 16 evenly spaced angles: 
azi = linspace (-pi, pi-pi/8, 16);

tgp = TransmitGridPolar(srcHgt, rcvHgt, range, azi);

\subsection{Impedance-plane model}

\subsubsection{Flat ground}

The impedance-plane propagation model, implemented in the KNEE package by the Java class mil. army . usace. easee. knee. ImpedancePlaneModel, solves for the sound field above a flat, impedance boundary (Attenborough et al. 1980). The atmosphere above the plane is assumed to be a homogeneous half-space, represented using the AirMedium class.

As discussed in the Background section, each model in EASEE has an associated Java class (or classes) defining the parameters needed by that model. A propagation model is created by first constructing the parameters and then constructing the propagation model using these parameters. Consider, for example, the following code:

params = ImpedancePlaneParamsHomo;

impModel = ImpedancePlaneModel (params);

or, more simply,

impModel = ImpedancePlaneModel (ImpedancePlaneParamsHomo);

Here we have constructed the parameters based on default values, which in this case correspond to impedance typical of grass-covered soil and dry air at room temperature and sea-level pressure. The Homo at the end of the ImpedancePlaneParamsHomo signifies that the parameters are constructed for a homogeneous environment (EnvironHomo). The impedance-plane model also has a parameter class called ImpedancePlaneParamsVertProf, which is for a vertical profile environment.

The following constructor for the impedance-plane model parameters passes the properties for the air and ground layers explicitly:

ImpedancePlaneParamsHomo(AcousticMedium refAir, AcousticMedium ground) 
Any valid AcousticMedium may be used for both the air and ground. However, only the phase speed, density, and attenuation of the air layer and the specific impedance of the ground layer will impact calculations made with the impedance-plane model. AirMedium is a nonabstract subclass of AcousticMedium and is generally used for the air layer refAir. Similarly, one of the porous-media models mentioned in section 6.2 (such as ZwikkerKostenMedium) is used for the ground.

The following constructor explicitly passes an EnvironHomo object, which may be constructed using the procedures described in section 5.2:

ImpedancePlaneParamsHomo (EnvironHomo scene)

In this version of the constructor, the surface temperature, humidity, and pressure values from this environment are used to construct the air properties, whereas the soil properties are used to construct the ground impedance. All other information in the EnvironHomo object is ignored.

Another useful approach to constructing parameters for the impedanceplane model uses predefined acoustical ground types and benchmark cases. The following code lists the available definitions:

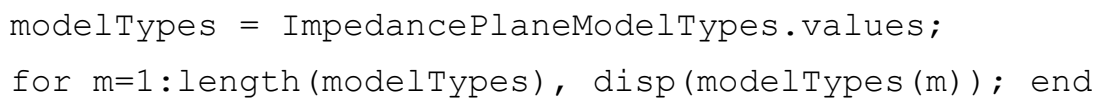

This results in

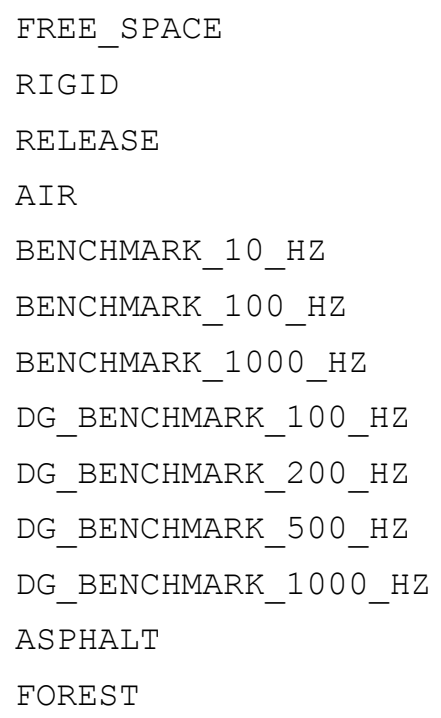


GRASS

GRAVEL

ICE

SAND

SNOW

WATER

Hence, to construct a model for a grass-covered ground surface, we would enter

params = ImpedancePlaneParamsHomo (modelTypes (14));

impModel = ImpedancePlaneModel (params);

In the previous listing of predefined acoustical ground types, the types starting with BENCHMARK _ are from Attenborough et al. (1995), whereas those starting with DG_BENCHMARK_ are from Di and Gilbert (1993).

The basic procedure for running the propagation model is to create a structured grid, which specifies the geometry of the problem, and then call the calculation using this grid and other input parameters, such as the acoustic frequency. Assuming a vertical grid has been constructed as described in section 6.3, we calculate and display the transmission loss using

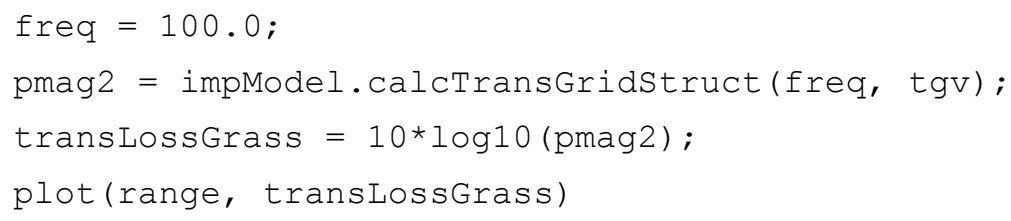

The key to the preceding code is the call to the calcTransGridstruct method, which takes two arguments, namely the frequency and the transmission grid, and returns the sound field for receiver positions on the specified grid. Note that the output of EASEE acoustical calculations is the squared magnitude of the pressure, normalized by the pressure that would be observed at $1 \mathrm{~m}$ in free space. Hence, we convert the output to decibels by taking ten times the base-ten algorithm. This quantity is often called the transmission loss, or TL. (Strictly speaking, it would make more sense to call the negative of this quantity the transmission loss, since a loss would then be positive. The TL as defined here might more properly be called a transmission gain. Nonetheless, this quantity is conventionally called the transmission loss.) 
To rerun the previous calculation with a rigid ground surface, we would enter

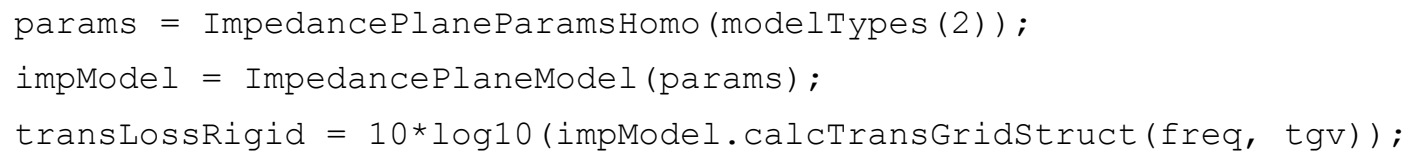

And to rerun the calculation for snow,

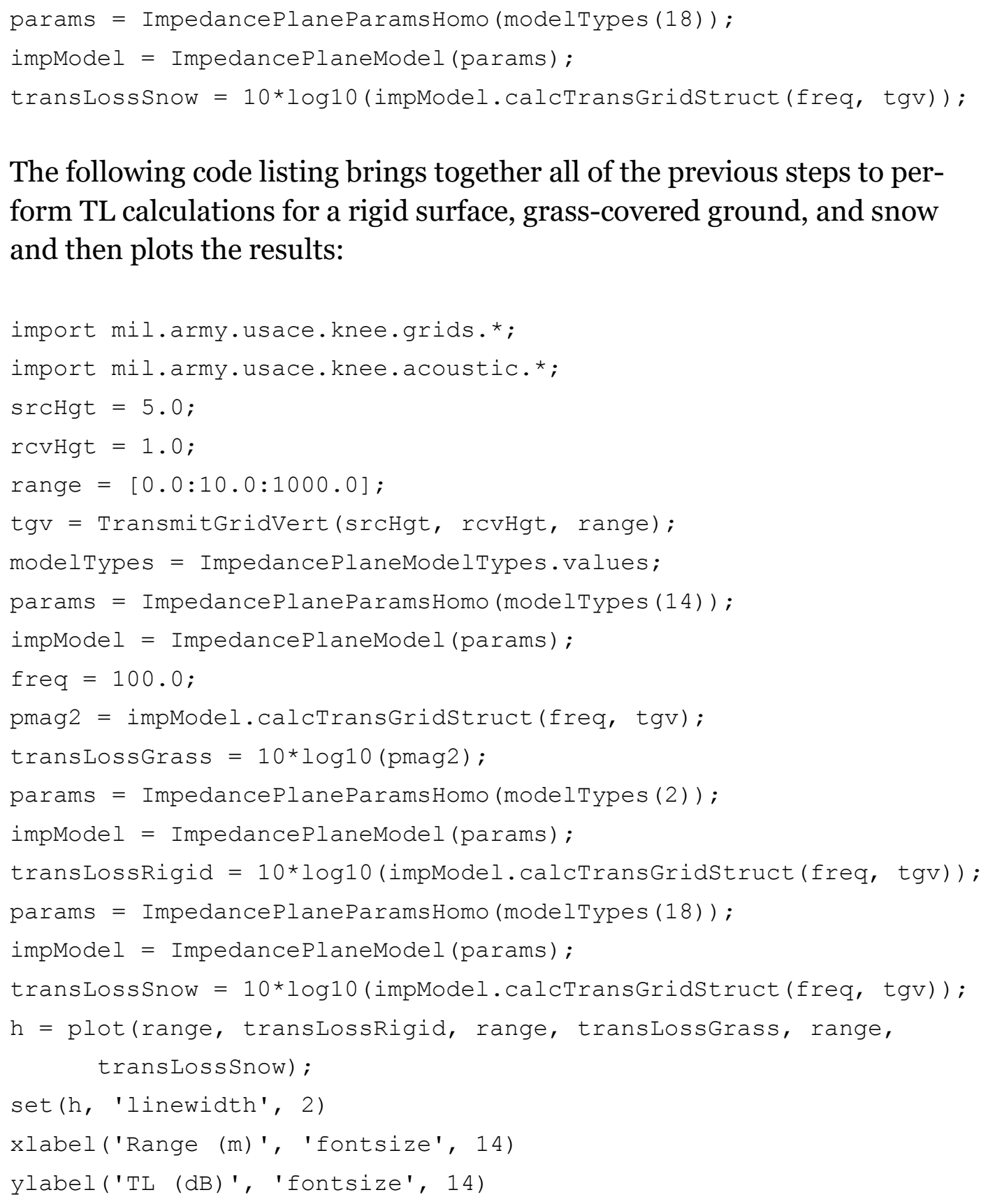


set(gca, 'fontsize', 14, 'linewidth', 2)

legend('rigid', 'grass', 'snow')

Figure 8 shows the resulting plot.

Figure 8. Transmission loss (TL) for propagation at a frequency of $100 \mathrm{~Hz}$ over several different ground surfaces.

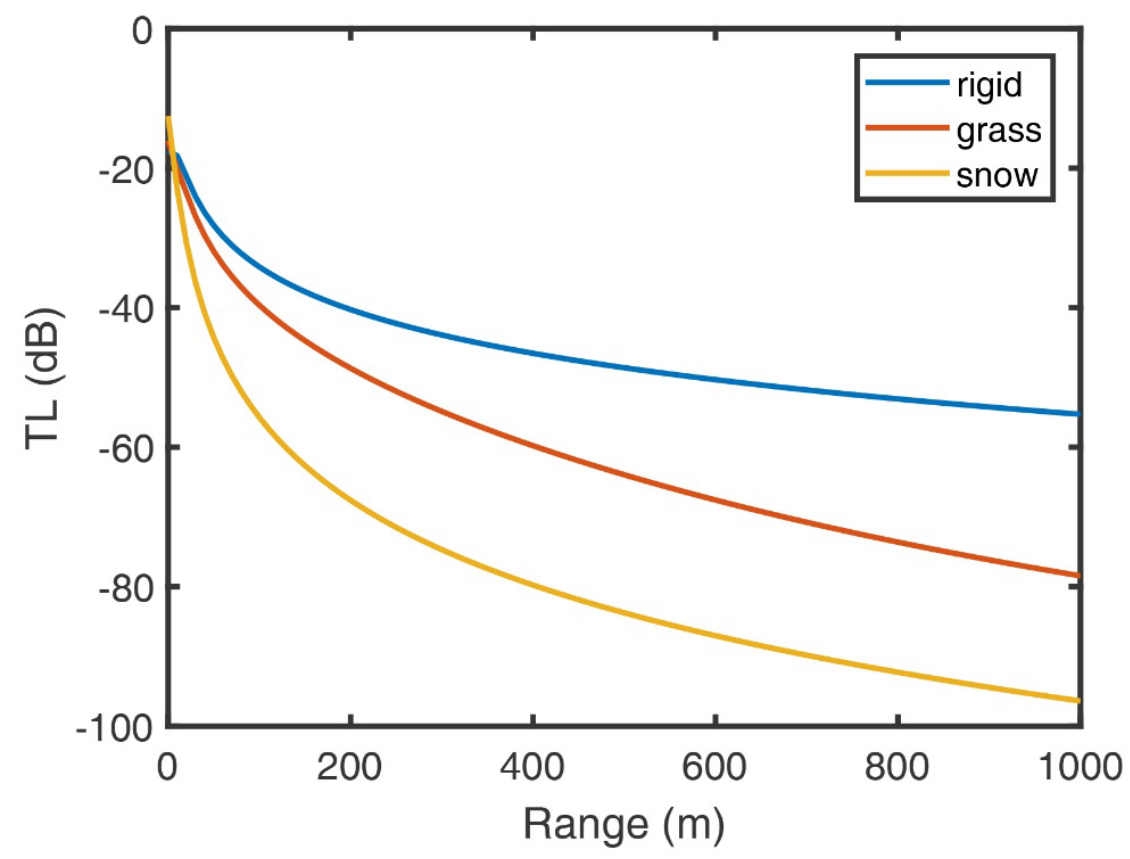

\subsubsection{Uneven ground}

The impedance-plane model can also be run in conjunction with a wedge terrain diffraction model (Wilson and Yamamoto 2014). This model, in effect, replaces the terrain between the source and receiver by a wedge shape that approximates the effect of the intervening terrain. Internally, EASEE runs the impedance-plane and diffraction models separately and then adds the loss due to the impedance ground to the loss due to terrain diffraction. The terrain diffraction calculation is turned on using the setTerraineffect method:

params.setTerrainEffect (true);

The terrain diffraction calculation requires a DEM. The DEM, as discussed earlier, is specified on a geographic grid. In this case, it is necessary to explicitly specify the geographic coordinates of the source and receiver. The calcTransGridGeo method is used for this purpose. This method has the following form: 
GeoGridCart2DDouble calcTransGridGeo(double freq, GeoCoord srcCoord, double srcHgt, GeoGridCart2D rcvGrid, double rcvHgt)

The input arguments are the frequency, a geographic coordinate for the horizontal source position, the source height, a geographic grid for the horizontal receiver position, and the receiver height. The output is a 2-D geographic grid, with a double [] [] data array, holding the squared pressure magnitude as calculated for each position in rcvGrid.

The following code listing illustrates how to perform a propagation calculation in complex terrain using the calcTransGridGeo method. In this example, the peaks function is used to create the terrain elevations, which is shown in Figure 9. The impedance-plane model, with terrain effects, is then called to calculate the TL, with the result shown in Figure 10.

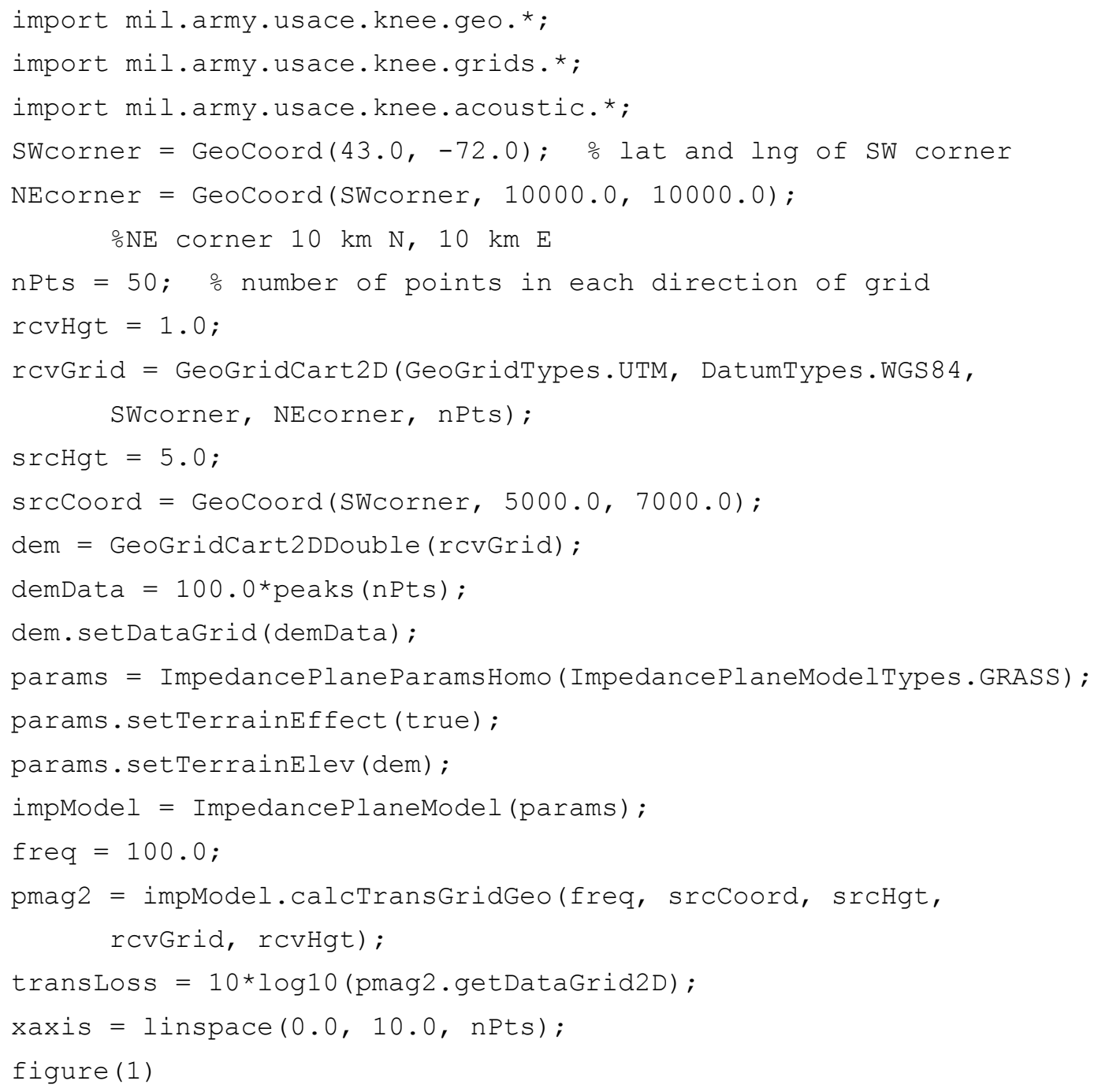




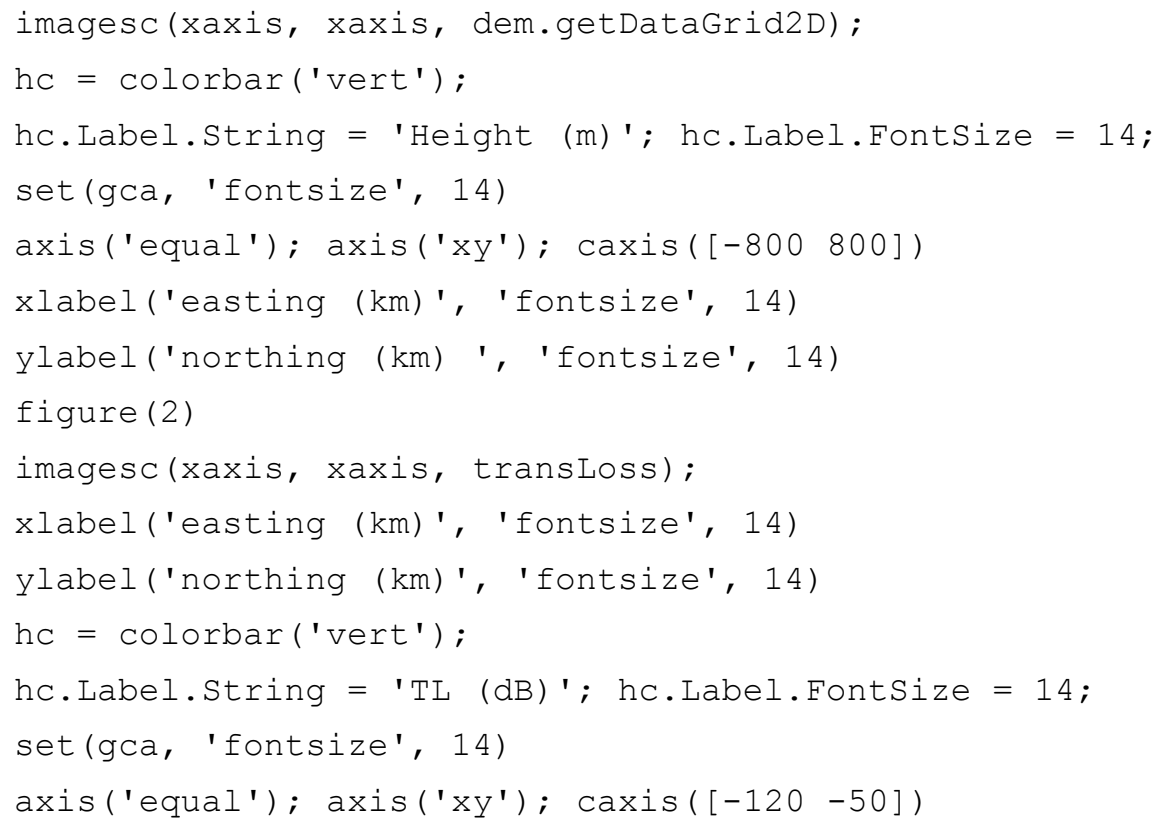

Figure 9. Terrain elevations (digital elevation model, or DEM) used for the transmission loss calculation shown in Fig. 10. The coordinate axes are the easting and northing relative to the southwest corner of the domain.

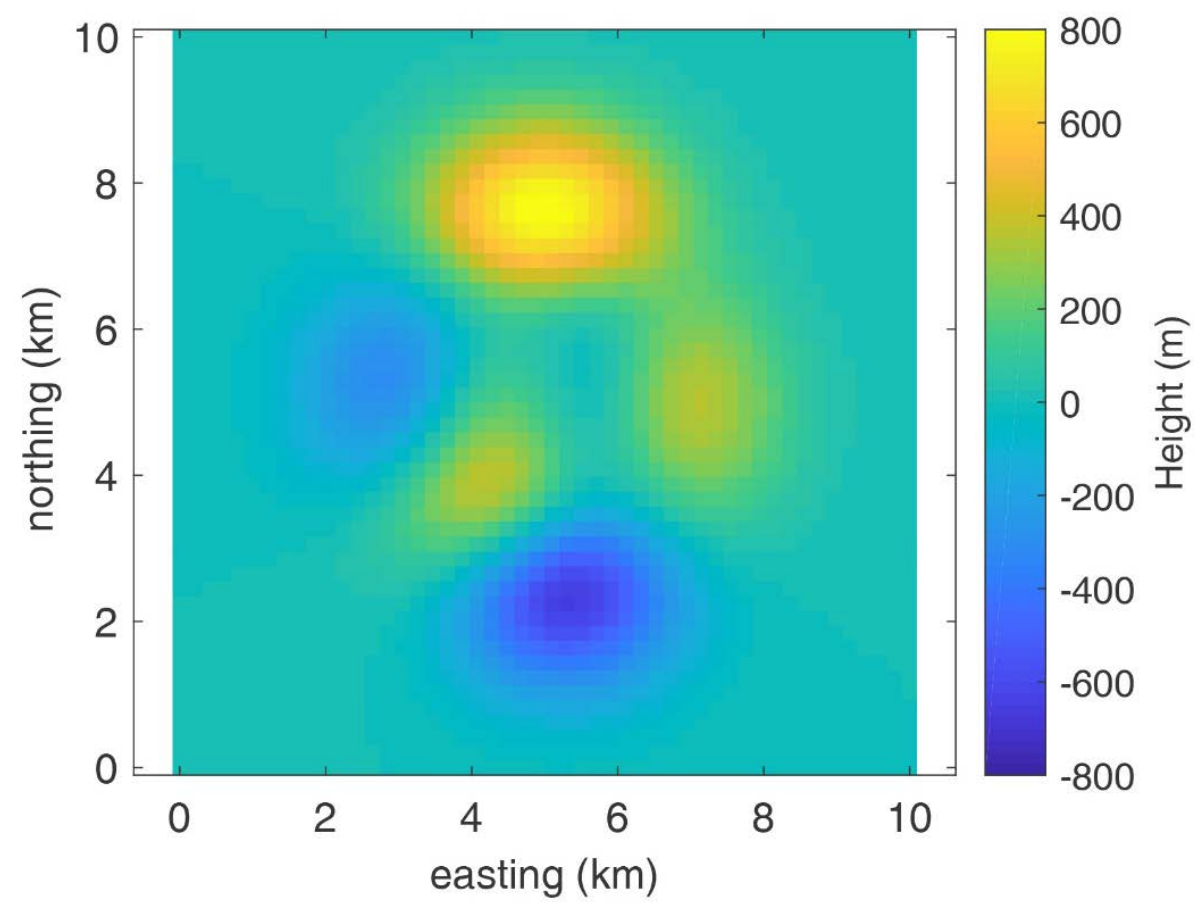


Figure 10. Transmission loss (TL) calculation for a source in hilly terrain. The frequency is $100 \mathrm{~Hz}$. The source is positioned at an easting of $7 \mathrm{~km}$ and a northing of $5 \mathrm{~km}$. The DEM for the calculation is shown in Fig. 9.

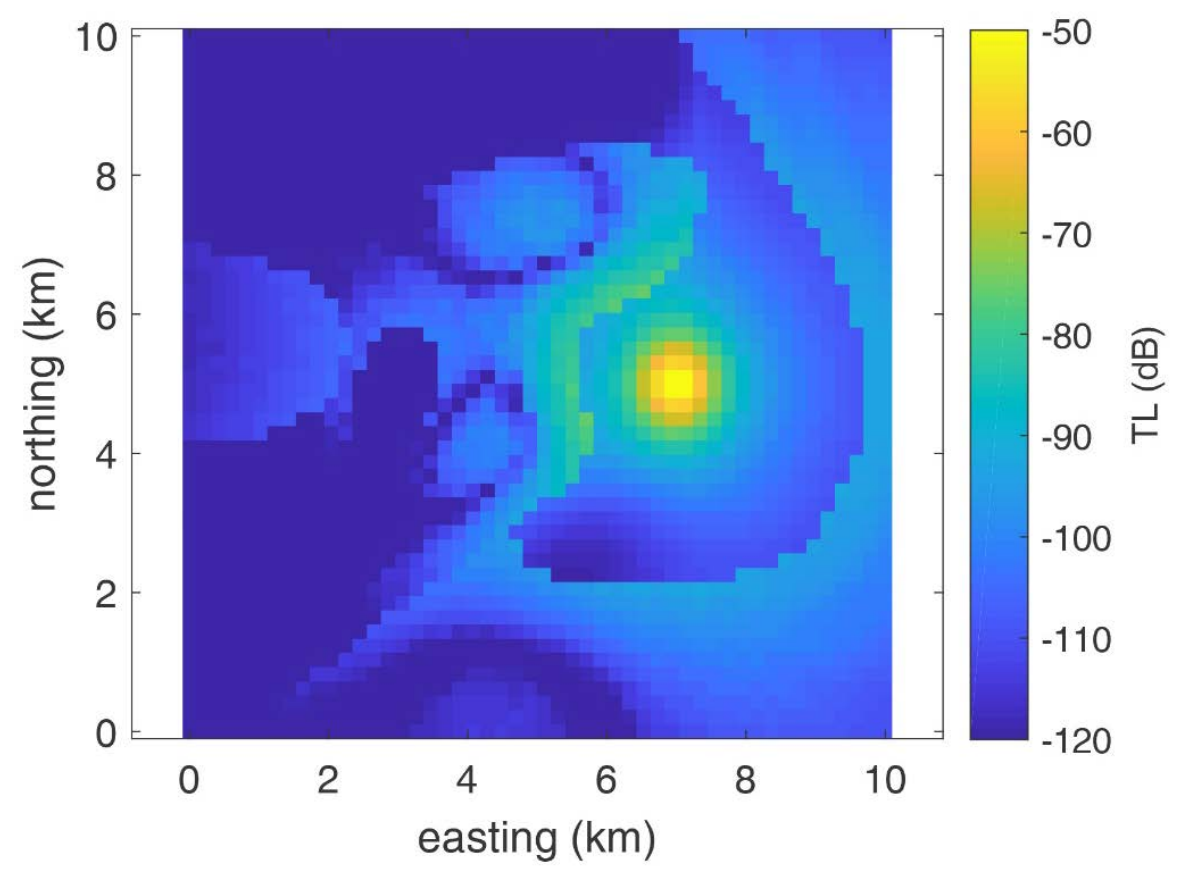

\subsection{Parabolic equation methods}

Parabolic equations (PE) efficiently calculate the impacts of weather and ground impedance on sound propagation in the atmosphere (Gilbert and Di 1993; Salomons 2001). Two PE codes are currently available in EASEE. These are Crank-Nicholson PE (CNPE), which is written in Java, and NASA's GFPE, which was originally written in FORTRAN and then compiled for Windows. Both are found in the EASEELib acoustic package.

The CNPE and GFPE share the same classes for defining parameters. Specifically, two classes are available, ParabolicEqParamsHome and ParabolicEqParamsVertProf. As the names of these classes suggest, they are used for homogeneous and vertical profile environments, respectively. The basic constructors are

and 
ParabolicEqParamsVertProf (EnvironVertProf scene)

For example, to create the parameters for a vertical profile calculation, enter

scene = EnvironVertProf;

params = ParabolicEqParamsVertProf(scene);

These classes also have methods to set the computational parameters. The setcomp method controls the parabolic approximation, range step, and height step:

setComp(boolean wideAngle, double delr_norm, double delz_norm)

The first argument specifies whether a wide-angle calculation is to be used (if available); the second and third arguments are the range and height steps, as normalized by the wavelength. By default, a narrow-angle calculation is performed with the step values both set to $1 / 10$ of a wavelength. For example, to set up a narrow-angle calculation with normalized range and height steps of $1 / 20$ of a wavelength, enter

params.setComp (false, 0.05, 0.05);

The following method controls the characteristics of the absorbing (sponge) layer at the top of the calculation domain:

setAbsLayer(double layerHgt, double layerThick)

The first of these arguments is the height at which the absorbing layer begins as a fraction of the maximum horizontal range in the calculation. For example, if the maximum horizontal range is $10 \mathrm{~km}$ and layerHgt is 0.1 , the absorbing layer will begin at $1 \mathrm{~km}$. The second argument is the thickness of the absorbing layer in wavelengths.

The CNPE and the GFPE currently handle only flat ground with constant ground properties. Both perform calculations using the polar transmission grid (TransGridpolar class) as described in section 6.3. 
Once the parameters and calculation grid have been constructed, it is straightforward to call either of the PE calculations. The name of the calculation class for the CNPE is CNParabolicEqn, whereas the GFPE is NASA3DG fpe. For example, to instantiate a CNPE calculation, enter

cnpe = CNParabolicEqn (params);

The following MATLAB code (which is a listing of a complete script) illustrates how to construct an environment (in this case, based on profiles constructed with MOST), run the CNPE and GFPE calculations, and then plot the results. For comparison purposes, we also run the impedanceplane model (section 6.4.1) and an alternative version of the CNPE available through the MATLAB interface (to be discussed in section 6.8).

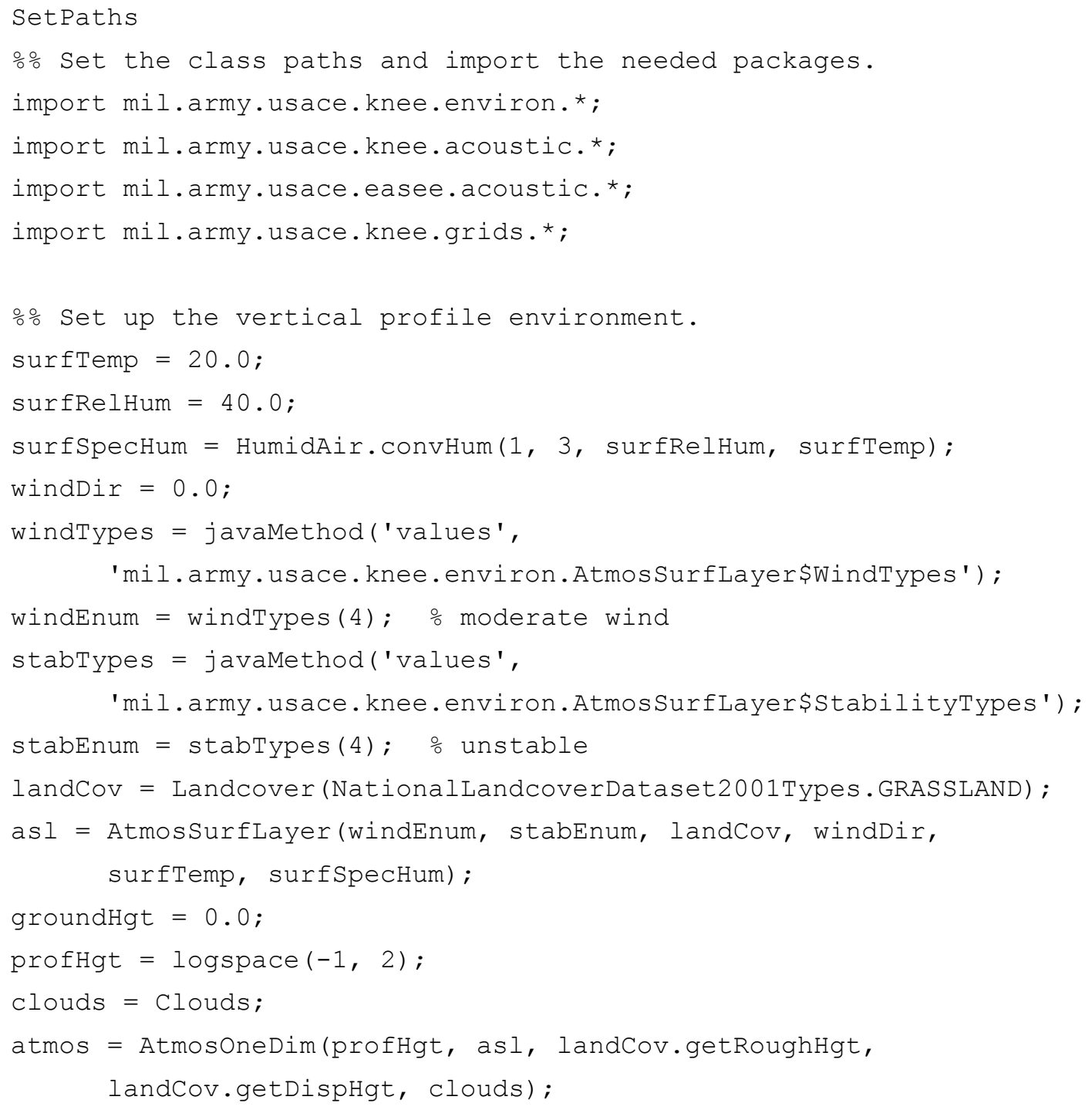




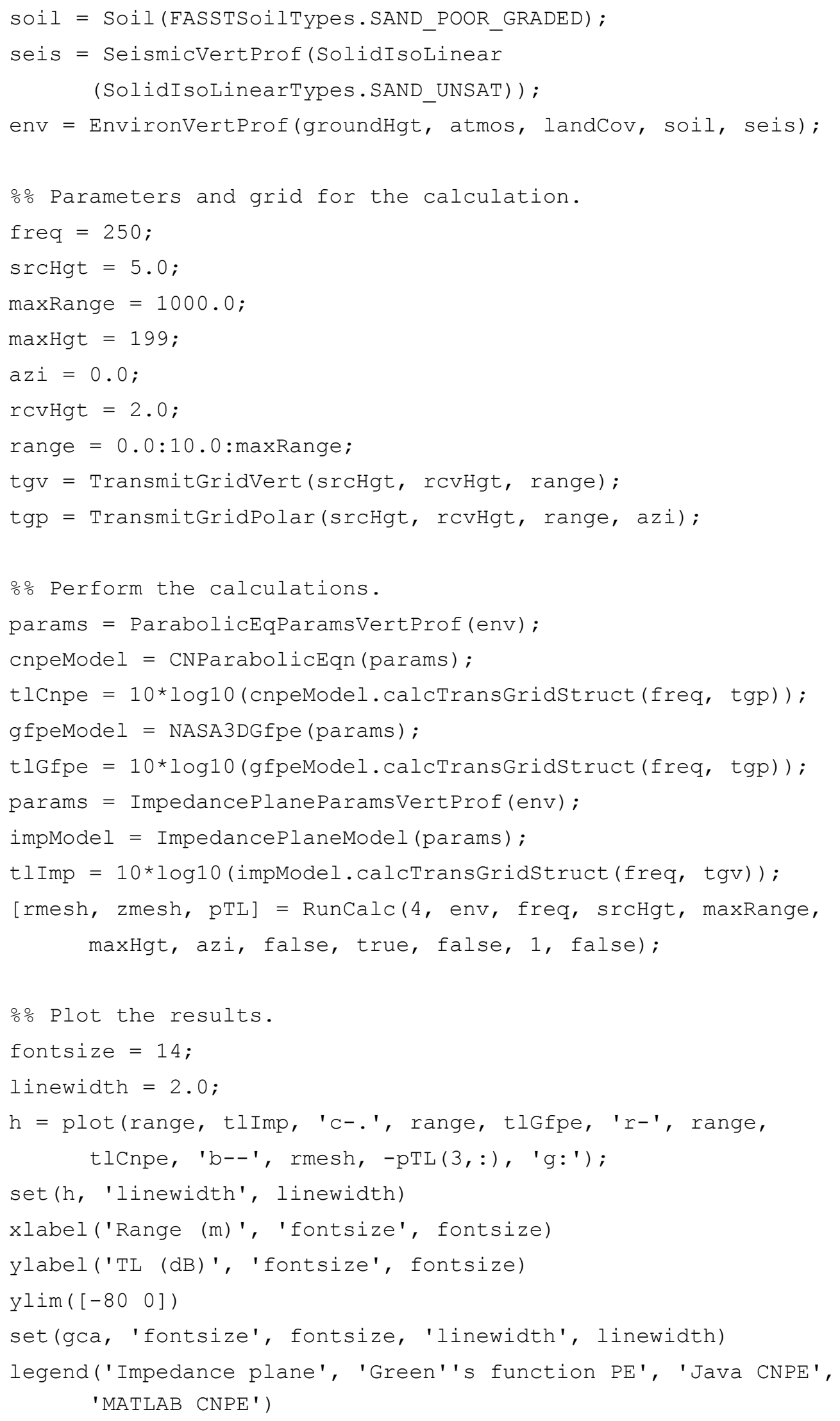


Figure 11 shows the resulting plot. At distances less than about $200 \mathrm{~m}$, the CNPEs and impedance-plane model are in close agreement. This is to be expected since the meteorological effects are not very large near to the source; hence, the impedance-plane model, which does not include the meteorology, gives a good result in this range. However, the GFPE differs substantially from the other calculations at these distances, even though previous works (e.g., Gilbert et al. 1990; Salomons 1998; Lihoreau et al. 2006) illustrate the accuracy of both the GFPE and other PE methods. This anomaly may occur because solutions of the GFPE may only come into agreement at the end of the first range step that separates applications of the Fourier transform. Thus, at these short distances $(<200 \mathrm{~m})$, the CNPE seems to be preferable to the GFPE.

For longer distances, greater than about $200 \mathrm{~m}$, the PE calculations all give nearly the same result. The predictions from the PEs differ substantially from the impedance-plane model due to the importance of the meteorological effects, in this case downward refraction in the downwind direction. The Java CNPE has a slightly smaller TL than the other two PEs (about $1 \mathrm{~dB}$ less). The reason for this discrepancy is unclear, but it is not very significant in a numerical sense.

Figure 11. Comparison of transmission loss (TL) calculations by several different codes.

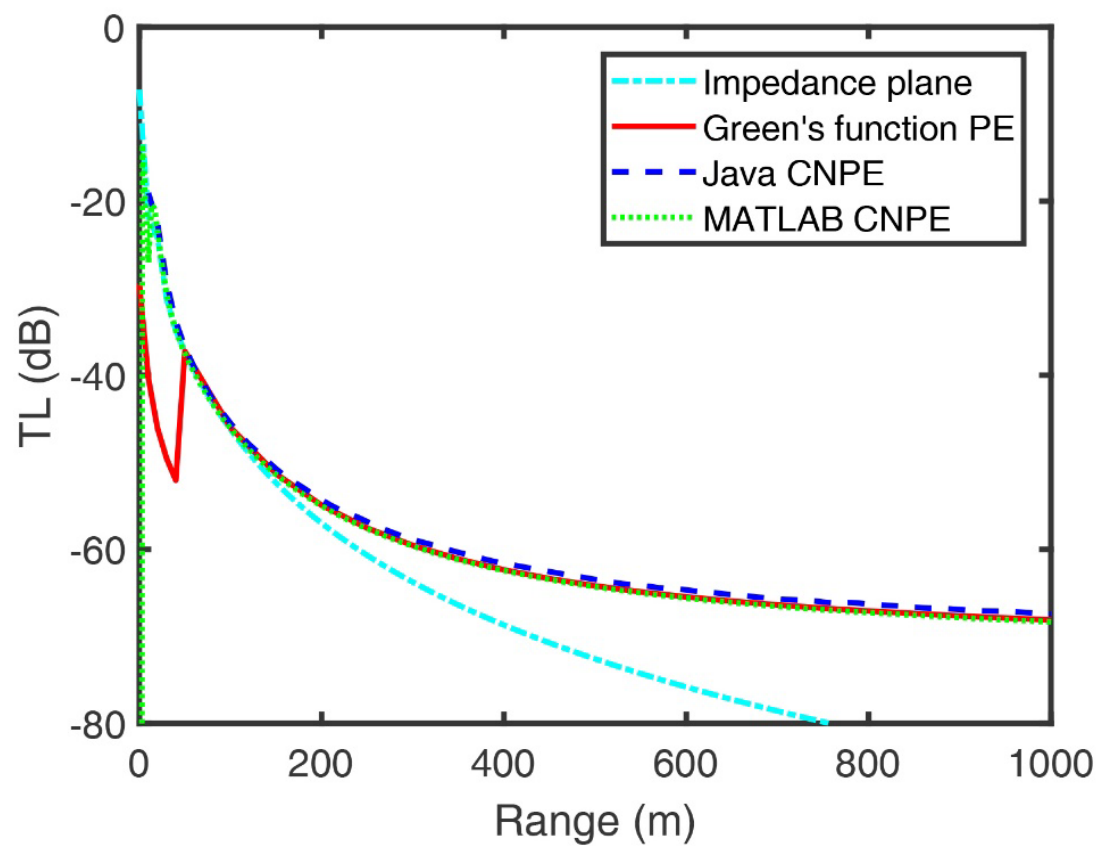




\subsection{BNOISE}

The ERDC Construction Engineering Research Laboratory's BNOISE (Blast Noise) model is based on look-up tables generated by a fast-field program (FFP) (Salomons 2001). The table look-up is relatively fast although the accuracy of the calculation depends on the availability of a table generated with profiles similar to those in EASEE's environmental object.

Use of BNOISE is very similar to the PEs. Classes for setting up the model parameters are available for the homogeneous and vertical profile environments, which are named BNOISEParams Homo and BNOISEParamsVertProf, respectively. The name of the actual calculation class is BNOISE. For example, once a vertical profile environment object and calculation grid have been constructed (by an approach such as that illustrated by the code listing in section 6.5), a BNOISE calculation can be run using

bnoiseModel = BNOISE (BNOISEParamsVertProf (env));

tlBnoise $=10 * \log 10$ (bnoiseModel.calcTransGridStruct(freq, tgp));

\subsection{Nord2000}

The Nord2000 model (Plovsing 2006) was originally developed for noisecontrol applications. Unlike the impedance-plane model, the PEs, and BNOISE, it is a heuristic (approximate) model rather than a numerical method for solving the wave equation or a variant thereof. However, Nord200o does have the advantage of being able to handle terrain diffraction and spatially varying ground-impedance.

Nord200o is run by the same general approach described for the other models. The classes for setting up the model parameters are Nord2000ParamsHomo and Nord2000ParamsVertProf, and the propagation model class is Nord2000Model. The Nord2000 model is imported using

import com.brrc.easee.acoustic. *;

\subsection{MATLAB acoustic propagation interface}

In addition to the propagation models available in EASEE, a number of models have been coded directly in MATLAB. An interface has also been developed to run these MATLAB models using the environmental representations in EASEE. This can be regarded as a hybrid approach to using 
EASEE in MATLAB; we use EASEE to define the inputs to the calculation but then run the actual calculation in MATLAB (rather than in Java).

The MATLAB models currently support only flat ground and a single type of land cover and soil. But several useful capabilities are provided that are unavailable in the Java code. For one, random turbulent fields, including temperature and wind velocity fluctuations, can be synthesized and incorporated into propagation calculations by using the parabolic equation (PE) and ray-tracing methods. This can be useful in cases of upward refraction, in which scattering by turbulence is particularly important (Wilson et al. 2015). Second, a capability exists for smoothly transitioning from the PE at narrow angles (within about $15^{\circ}-20^{\circ}$ of horizontal, where the $\mathrm{PE}$ is accurate) to an impedance-plane model at high angles (where the PE is inaccurate). The MATLAB interface also includes a ray-tracing calculation, which can be overlaid on the TL calculation if desired.

The MATLAB interface involves two primary functions (m-files): RunCalc.m and PlotCalc.m. These functions run the propagation calculation and plot the output, respectively. The header for RunCalc.m, which shows the input and output arguments, is

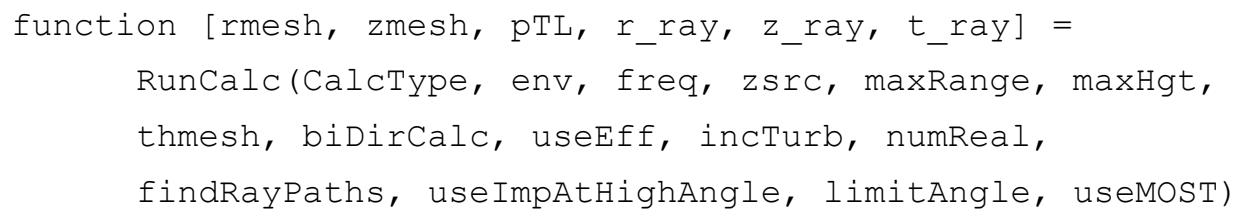

The first input argument, CalcType, specifies the method for calculating the TL. The allowed values are $1=$ impedance plane model, $2=$ ray tracing, 3 = fast-field program, $4=$ narrow-angle PE, and $5=$ wide-angle PE. The second argument is an EnvironvertProf object constructed using EASEE, which specifies the atmospheric vertical profiles and ground properties.

The remaining input arguments are as follows:

- freq: acoustic frequency $(\mathrm{Hz})$

- zsrc: source height (m)

- maxRange: maximum horizontal distance (m)

- maxHgt: maximum height (m)

- thmesh: azimuthal angles at which the calculation will be performed (deg) 
- bidirCalc: true to perform a calculation at thmesh and at thmesh + pi, where the calculations are joined together with the source at zero range

- useEff : true to use the effective sound-speed approximation

- incTurb: true to include random atmospheric turbulence

- numReal: number of turbulence realizations to include prior to averaging

- findRayPaths: true if ray paths are also to be calculated and overlaid on the TL display

- useImpAtHighAngle: true if the impedance plane model is to be substituted for high elevation angles when a PE calculation is used

- limitangle: elevation angle (deg) above which the impedance model is to be used (when useImpAtHighAngle=true)

- usemost: use the Monin-Obukhov Similarity Theory for the profiles (regardless of whether MOST was used to construct the profiles in the EnvironVertProf object)

The input arguments starting with birDirCalc are all optional, in which case they will be set to defaults (biDirCalc $=$ false, useEff $=$ false, incTurb = false, numReal = 1 , findRayPaths = false, useImpAtHighAngle = false, limitAngle = 30.0, useMoST = false).

The output arguments of RunCalc are as follows:

- rmesh: horizontal coordinate mesh for TL

- zmesh: vertical coordinate mesh for TL

- pTL: TL (in decibels relative to a distance of $1 \mathrm{~m}$ from the source)

- r_ray: horizontal coordinates of rays

- z_ray: vertical coordinates of rays

- t_ray: time coordinates of rays

The final three arguments are empty if no ray-tracing calculation was requested (i.e., CalcType was set to 2 and findRayPaths was set to false).

The header for PlotCalc. $m$ is

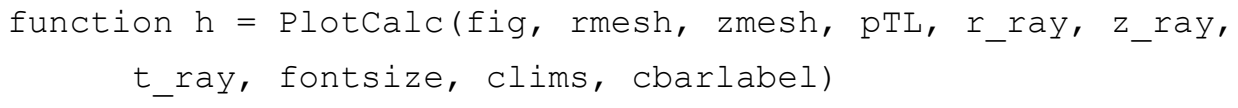

Here, $f i g$ is the number of the figure window for the plot. If an empty array is passed, a new figure window will be opened. The next six inputs 
match the output of the PlotCalc function. The argument fontsize specifies the font size to be used for the axis labels; clims specifies the color limits; and cbarlabel specifies the label for the color bar. These last three arguments can be omitted, in which case they will be set to defaults (12, [30 90], and 'TL (dB) ', respectively). The single output argument $h$ is a handle to the axes of the plot, which can be used to tailor other graphics properties as desired.

The following code, which is a complete MATLAB script, illustrates how to construct an environment, run a wide-angle parabolic equation calculation through the MATLAB interface, and then plot the results as a 2-D image (vertical plane). Figure 12 shows the plot that results from running this code:

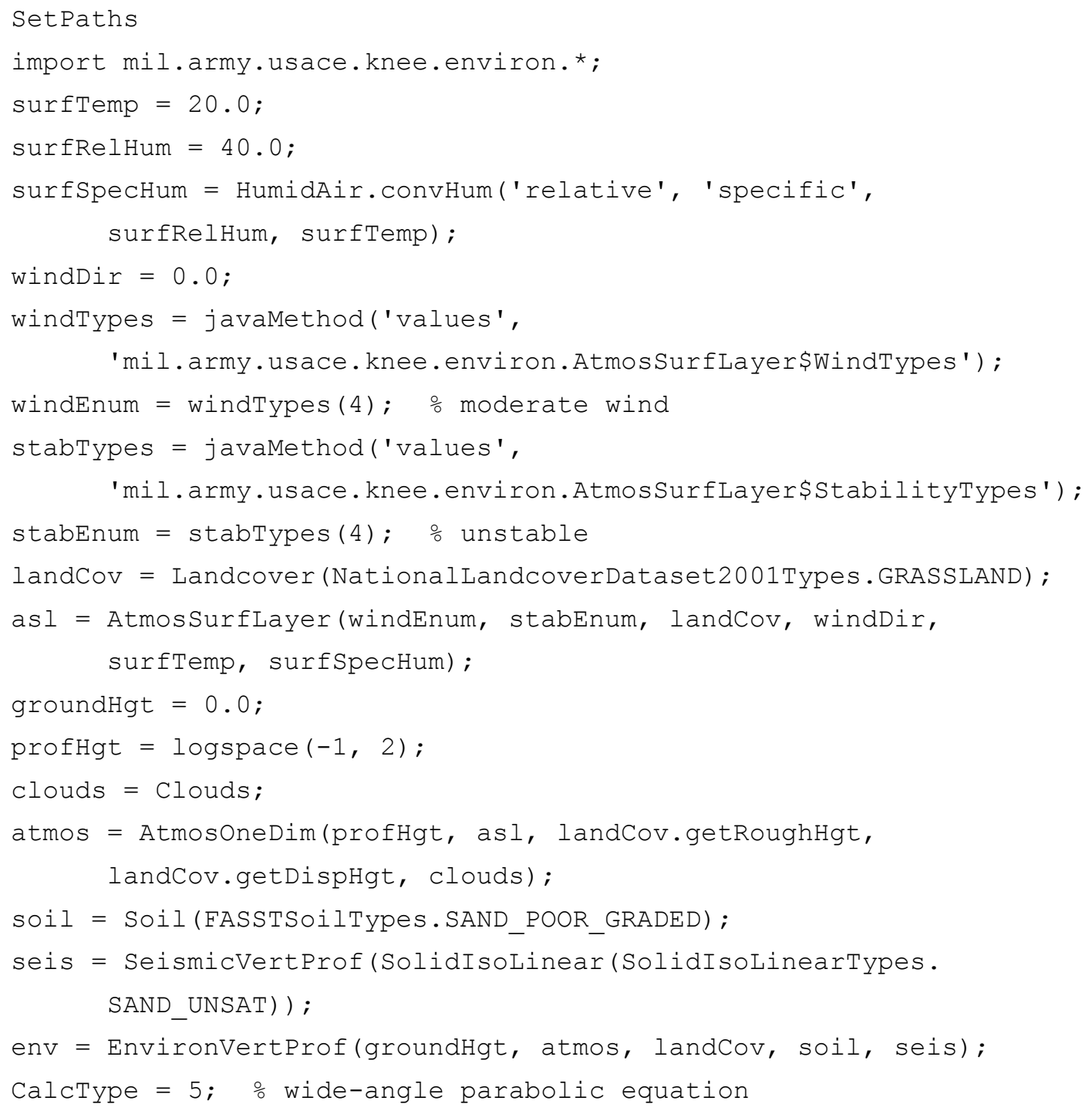




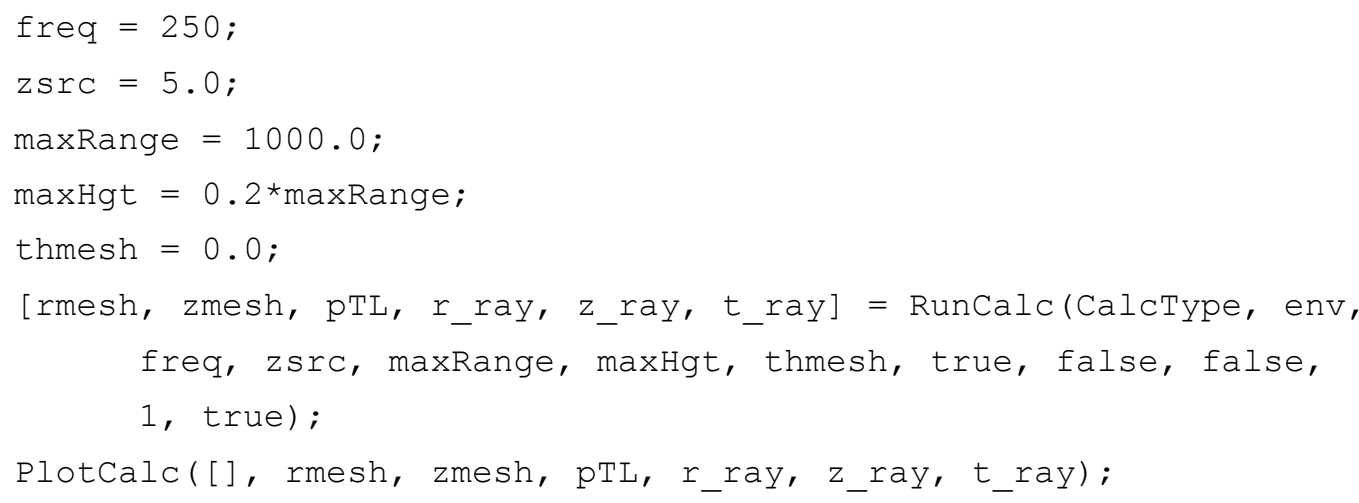

Figure 12. Transmission loss resulting from a wide-angle parabolic equation calculation using the MATLAB interface.

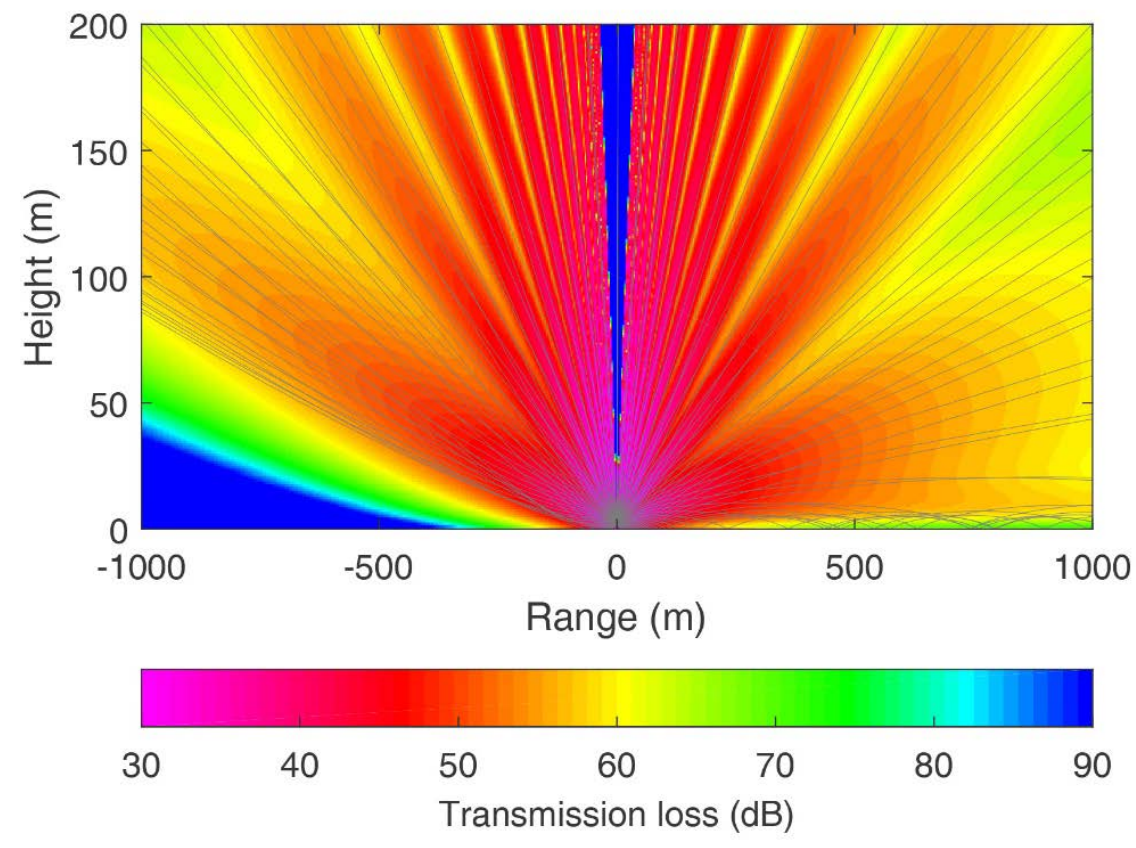




\section{Full Example Script for Testing EASEE in MATLAB}

The following example script-entitled calcExampWithWRF.m-can be copied and pasted into MATLAB to test EASEE and its functionality with data from the WRF weather model. It is also located in the folder where StartEASEE.m resides. It produces many of the plots shown previously and two additional plots (Figures 13 and 14) that illustrate the difference in TL between simulations that include and exclude WRF weather model data.

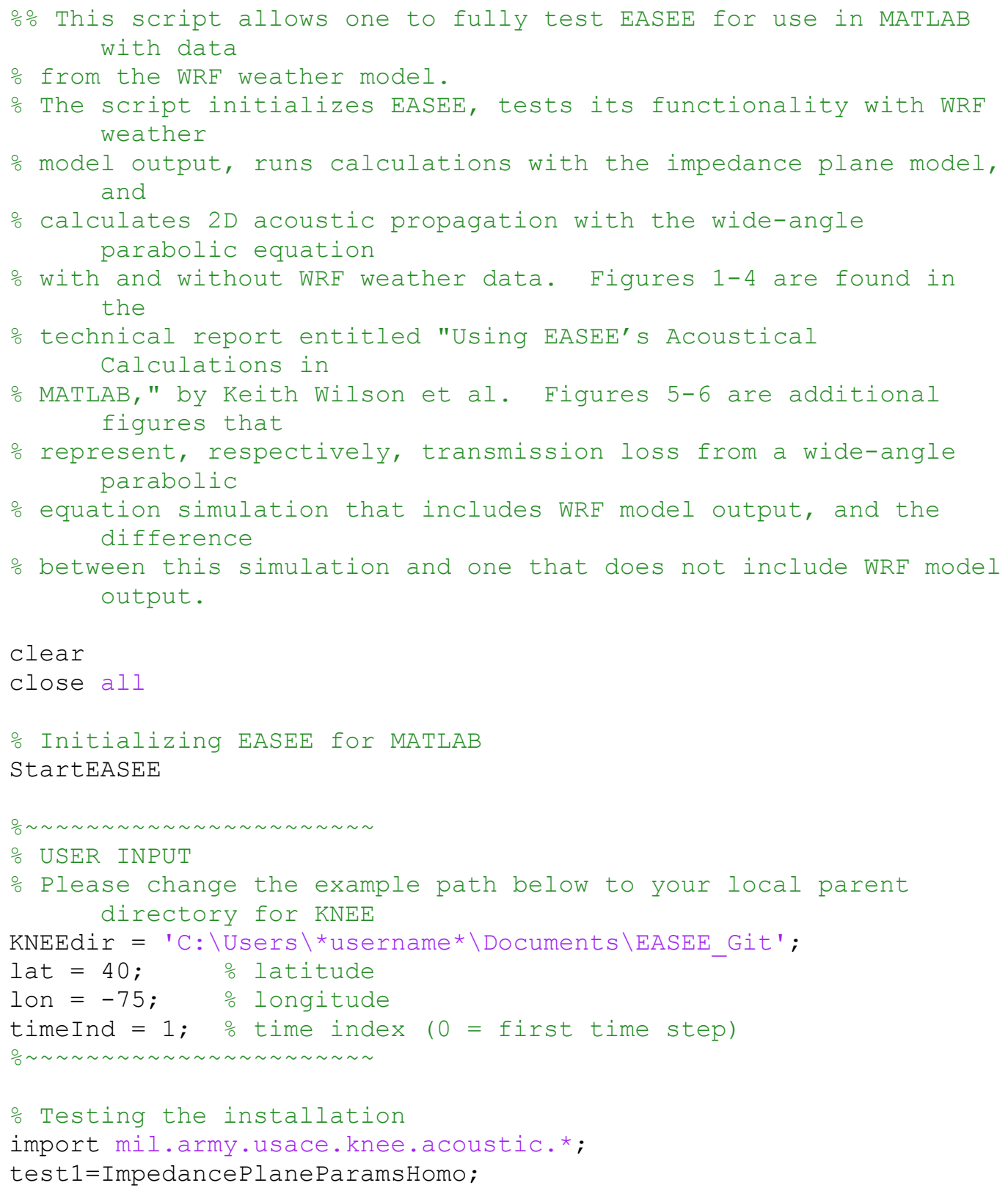




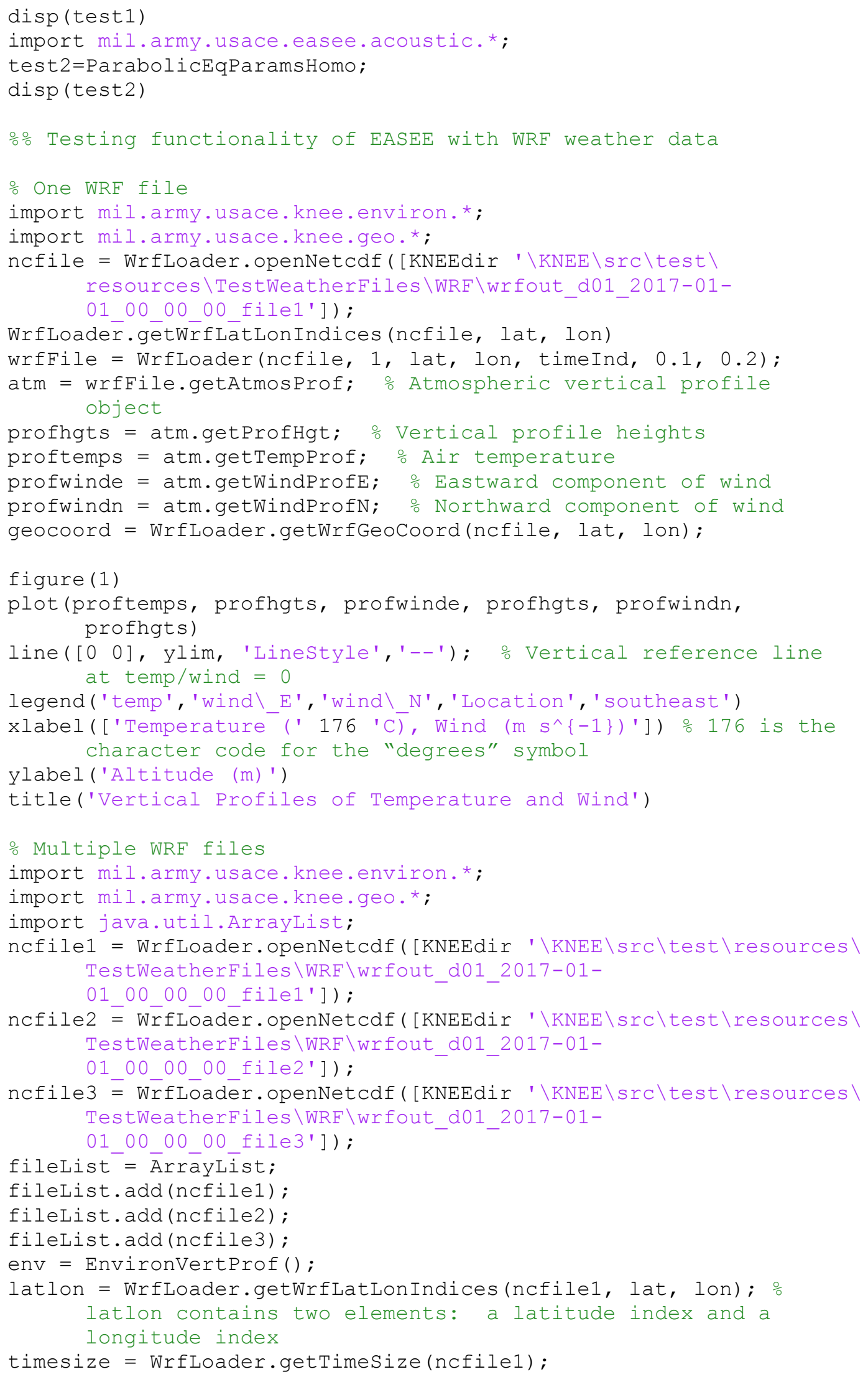




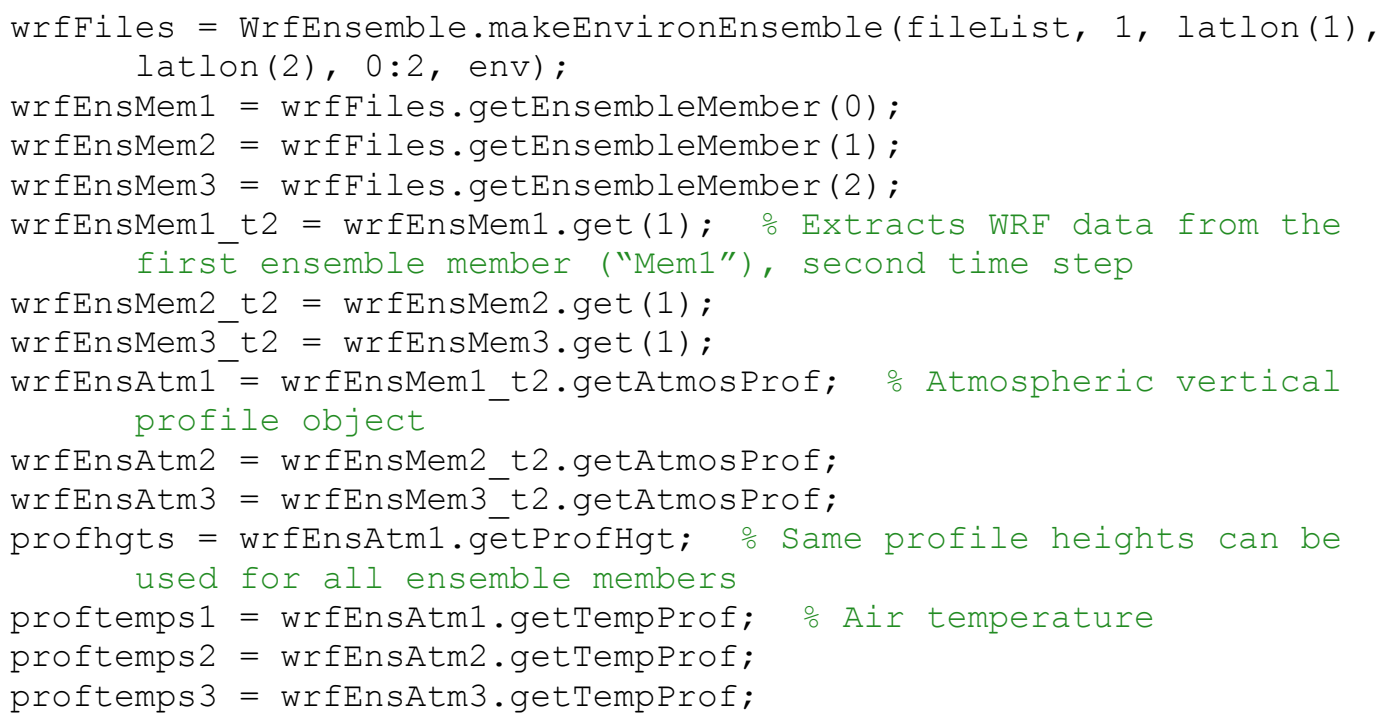




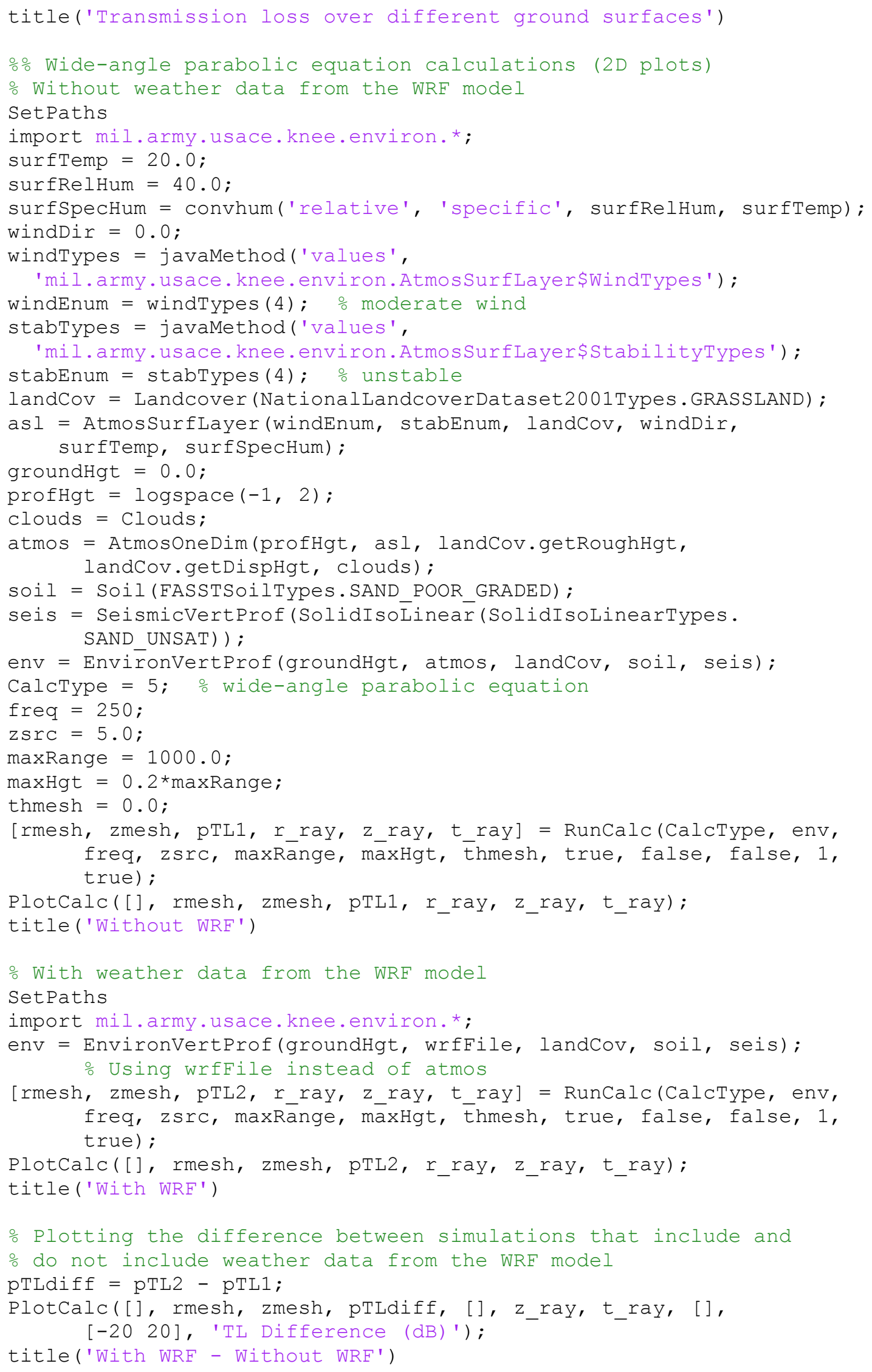


Figure 13. Same as Fig. 12 but using atmospheric output from the WRF weather model.

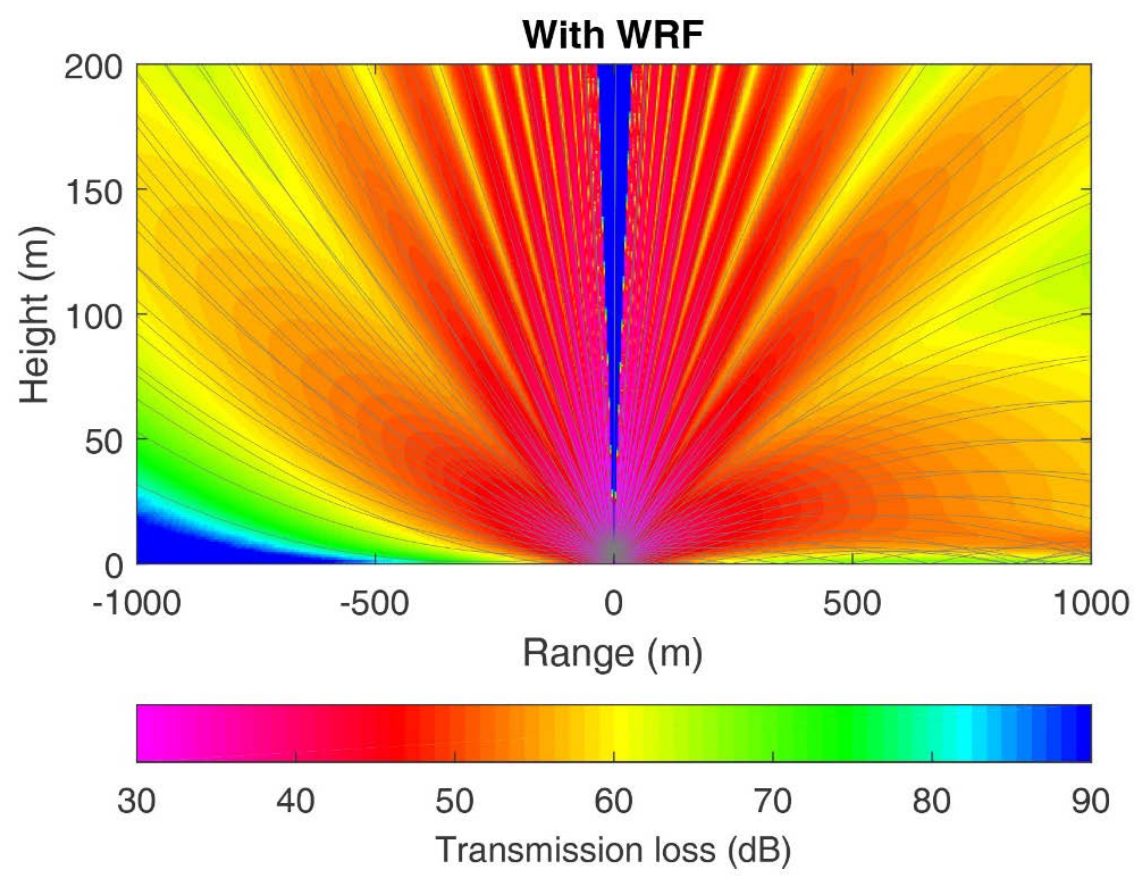

Figure 14. Difference in transmission loss (TL) between Fig. 13 and Fig. 12.

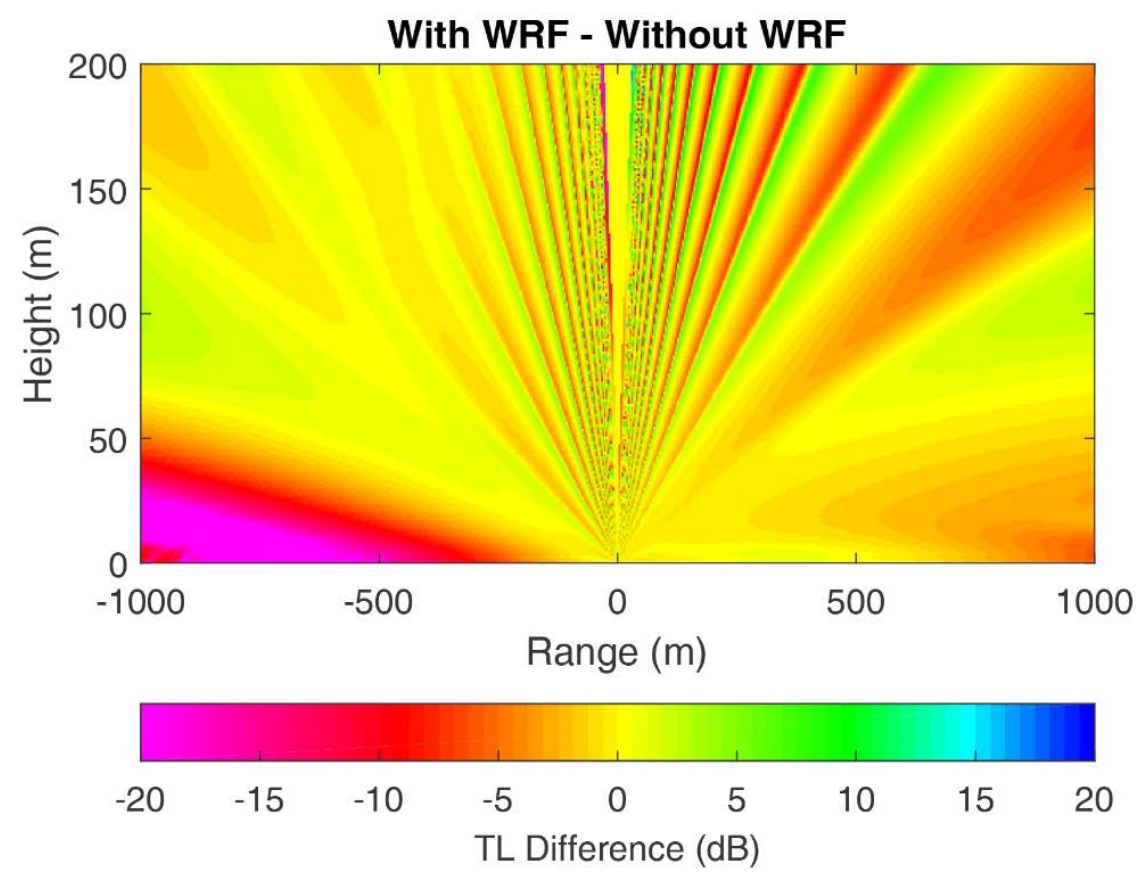




\section{Conclusion}

This report has shown how EASEE can provide a powerful MATLAB toolbox for creating representations of the atmosphere and terrain and for calculating sound propagation based on these representations. This toolbox is potentially useful for many projects involving acoustics and noise control.

We described two general approaches, both of which involved using EASEE to represent the propagation environment (atmosphere and terrain): In one approach, we used EASEE to perform the sound propagation calculations, while in the other, we used MATLAB. Both approaches are potentially useful, depending on whether the user wishes to code their propagation algorithms in Java or MATLAB.

We envision follow-ons to this effort in two main directions. First, the atmospheric representations are already being extended to $3-\mathrm{D}$, and it would be desirable to have a library of sound propagation models that can perform calculations with these 3-D data. Second, toolboxes for other signal modalities, such as radio frequency (RF), visible, and infrared (IR) propagation could be created.

Overall, this MATLAB interface can be helpful for those would would like to access the already numerous signal modeling capabilities of EASEE, and its utility will continue to improve as more features are added to EASEE in future versions. 


\section{References}

Attenborough, K., S. I. Hayek, and J. M. Lawther. 1980. Propagation of Sound over a Porous Half-Space. Journal of the Acoustical Society of America 68 (5): 14931501. https://doi.org/10.1121/1.385074.

Attenborough, K., S. Taherzadeh, H. E. Bass, X. Di, R. Raspet, G. R. Becker, A. Güdesen, A. Chrestman, G. A. Daigle, A. L'Espérance, Y. Gabillet, K. E. Gilbert, Y. L. Li, M. J. White, P. Naz, J. M. Noble, and H. A. J. M. van Hoof. 1995. Benchmark Cases for Outdoor Sound Propagation Models. Journal of the Acoustical Society of America 97 (1): 173-191. https://doi.org/10.1121/1.412302.

Di, X., and K. E. Gilbert. 1993. An Exact Laplace Transform Formulation for a Point Source above a Ground Surface. Journal of the Acoustical Society of America 93 (2): 714-720. https://doi.org/10.1121/1.405435.

Eckel, B. 2006. Thinking in Java. 4th ed. Upper Saddle River, NJ: Prentice Hall.

Frankenstein, S., and G. G. Koenig. 2004. Fast All-Season Soil Strength (FASST). ERDC/CRREL SR-04-1. Hanover, NH: U.S. Army Engineer Research and Development Center.

Gilbert, K. E., R. Raspet, and X. Di. 1990. Calculation of Turbulence Effects in an Upward-Refracting Atmosphere. Journal of the Acoustical Society of America 87 (6): 2428-2437. https://doi.org/10.1121/1.399088.

Gilbert, K. E., and X. Di. 1993. A Fast Green's Function Method for One-Way Sound Propagation in the Atmosphere. Journal of the Acoustical Society of America 94:2343-2352. https://doi.org/10.1121/1.407454.

Lihoreau, B., B. Gauvreau, M. Bérengier, P. Blanc-Benon, and I. Calmet. 2006. Outdoor Sound Propagation Modeling in Realistic Environments: Application of Coupled Parabolic and Atmospheric Models. Journal of the Acoustical Society of America 120 (1): 110-119. https://doi.org/10.1121/1.2204455.

Lowe, D., and B. Burd. 2007. Java All-in-One Desk Reference for Dummies. 2nd ed. Indianapolis, IN: Wiley Publishing, Inc.

MathWorks. 2018a. How do I change the Java Virtual Machine (JVM) that MATLAB is using on Windows? MATLAB Answers. Last modified 9 May 2018. https://www.mathworks.com/matlabcentral/answers/130359-how-do-i-change-the-javavirtual-machine-jum-that-matlab-is-using-on-windows.

MathWorks. 2018b. How do I change the Java Virtual Machine (JVM) that MATLAB is using on macOS? MATLAB Answers. Last modified 13 June 2018. https://www.mathworks.com/matlabcentral/answers/103056-how-do-i-change-the-javavirtual-machine-jum-that-matlab-is-using-on-macos.

NCAR (National Center for Atmospheric Research). 2017. The Weather Research and Forecasting Model. https://www.mmm.ucar.edu/weather-research-and-forecasting-model (accessed 2 June 2017). 
NCAR (National Center for Atmospheric Research). 2018. WRF Model Users' Page. http://www2.mmm.ucar.edu/wrf/users// (accessed 26 December 2018).

Oracle. n.d. Java SE Downloads. http://www.oracle.com/technetwork/java/javase/downloads.

Perry, S. J. 2010. Introduction to Java Programming, Part 1: Java Language Basics, Object-Oriented Programming on the Java Platform. IBM Developer. https://www.ibm.com/developerworks/java/tutorials/i-introtojava1/index.html.

Plovsing, B. 2006. Nord20oo. Validation of the Propagation Model. Delta Acoustics AV 1117/o6. Hørsholm, Denmark: Danish Electronics, Light \& Acoustics.

Rouse, M. 2014. Data Abstraction Layer. WhatIs.com. Newton, MA: TechTarget. https://whatis.techtarget.com/definition/database-abstraction-layer.

Salomons, E. M. 1998. Improved Green's Function Parabolic Equation Method for Atmospheric Sound Propagation. Journal of the Acoustical Society of America 104 (1): 100-111. https://doi.org/10.1121/1.423260.

Salomons, E. M. 2001. Computational Atmospheric Acoustics. Dordrecht, the Netherlands: Kluwer Academic.

Sierra, K., and B. Bates. 2005. Head First Java. 2nd ed. Sebastopol, CA: O’Reilly Media, Inc.

Skamarock, W. C., J. B. Klemp, J. Dudhia, D. O. Gill, D. M. Barker, M. G. Duda, X.-Y. Huang, W. Wang, and J. G. Powers. 2008. A Description of the Advanced Research WRF Version 3. NCAR/TN-475+STR. Boulder, CO: National Center for Atmospheric Research.

Stull, R. B. 1988. An Introduction to Boundary Layer Meteorology. Boston: Kluwer Academic Publishers.

Wilson, D. K., J. T. Kalb, N. Srour, T. Pham, and B. M. Sadler. 2002. Sensor Algorithm Interface and Performance Simulations in an Acoustical Battlefield Decision Aid. ARL-TR-2860. Adelphi, MD: U.S. Army Research Laboratory.

Wilson, D. K., R. Bates, and K. K. Yamamoto. 2009. Object-Oriented Software Model for Battlefield Signal Transmission and Sensing. ERDC/CRREL TR-09-17. Hanover, NH: U.S. Army Engineer Research and Development Center.

Wilson, D. K., and K. K. Yamamoto. 2014. Environmental Awareness for Sensor and Emitter Employment (EASEE): Software Design Version 2. ERDC/CRREL TR14-27. Hanover, NH: U.S. Army Engineer Research and Development Center.

Wilson, D. K., C. L. Pettit, and V. E. Ostashev. 2015. Sound Propagation in the Atmospheric Boundary Layer. Acoustics Today 11 (2): 44-53. 


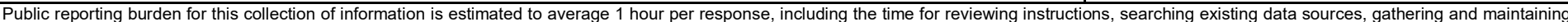

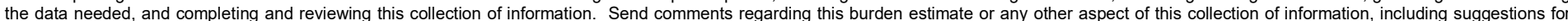

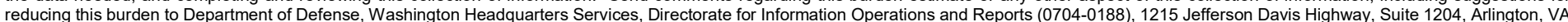

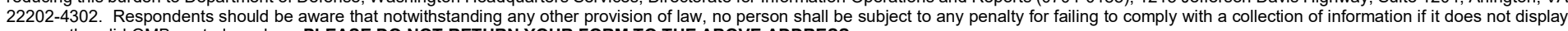
a currently valid OMB control number. PLEASE DO NOT RETURN YOUR FORM TO THE ABOVE ADDRESS.
1. REPORT DATE (DD-MM-YYYY)
January 2019

\section{Technical Report/Final}

\section{TITLE AND SUBTITLE}

\section{Using EASEE's Acoustical Calculations in MATLAB}

\section{AUTHOR(S)}

D. Keith Wilson, Ross E. Alter, Katrina M. Burch, and Michelle E. Swearingen

\section{DATES COVERED (From - To)}

\section{5a. CONTRACT NUMBER}

5b. GRANT NUMBER

5c. PROGRAM ELEMENT NUMBER

62784/T40/46

5d. PROJECT NUMBER

5e. TASK NUMBER

\section{5f. WORK UNIT NUMBER}

8. PERFORMING ORGANIZATION REPORT NUMBER

ERDC TR-19-1

. Army Engineer Research and Development Center (ERDC)

Cold Regions Research and Engineering Laboratory (CRREL)

72 Lyme Road

Hanover, NH 03755-1290

9. SPONSORING / MONITORING AGENCY NAME(S) AND ADDRESS(ES)

Headquarters, U.S. Army Corps of Engineers

Washington, DC 20314-1000

11. SPONSOR/MONITOR'S REPORT NUMBER(S)

\section{DISTRIBUTION / AVAILABILITY STATEMENT}

Approved for public release; distribution is unlimited.

\section{SUPPLEMENTARY NOTES}

ERDC 6.2/6.3 Military Engineering (ME) RAFTER

\section{ABSTRACT}

EASEE (Environmental Awareness for Sensor and Emitter Employment) is a Java-based software framework for modeling the impacts of the weather and terrain on signal propagation and sensor performance. EASEE includes extensive capabilities for representing the environment (atmosphere, land cover, terrain elevation, and soil properties), along with many different models related to acoustic, optical, radio frequency, and seismic signals. This report describes how to run EASEE from MATLAB, which is a popular software package for performing numerical calculations and displaying graphics. For this purpose, a simple installation configuration and MATLAB script were devised to set up and initialize EASEE. The focus of the report is on using EASEE for acoustic propagation calculations, which is its most mature signal modality. The report describes two general approaches to performing acoustical calculations in EASEE: one that involves using EASEE for its environmental representation only and then running the acoustic propagation calculation using a MATLAB toolbox and a second that uses EASEE for both its environmental layer and the acoustical calculation. Overall, this report shows that the MATLAB user interface provides convenient access to EASEE's powerful signal modeling capabilities.

\section{SUBJECT TERMS}

EASEE (Computer program), Computer software, Detectors--Environmental aspects, Detectors--Computer simulation, Remote sensing--Atmospheric effects, Digital communications

\section{SECURITY CLASSIFICATION OF:}

\section{a. REPORT}

Unclassified

\section{b. ABSTRACT}

Unclassified

\section{c. THIS PAGE}

Unclassified
17. LIMITATION OF ABSTRACT

SAR
18. NUMBER OF PAGES

106 19a. NAME OF RESPONSIBLE PERSON

19b. TELEPHONE NUMBER (include area code) 